\title{
Star formation in the local Universe from the CALIFA sample
}

\section{Calibrating the SFR using integral field spectroscopy data ${ }^{\star}$}

\author{
C. Catalán-Torrecilla ${ }^{1}$, A. Gil de Paz ${ }^{1}$, A. Castillo-Morales ${ }^{1}$, J. Iglesias-Páramo ${ }^{2,3}$, S.F. Sánchez ${ }^{4}$, R. C. Kennicutt ${ }^{5}$, \\ P.G. Pérez-González ${ }^{1}$, R. A. Marino ${ }^{6}$, C.J. Walcher $^{7}$, B. Husemann ${ }^{8}$, R. García-Benito ${ }^{2}$, D. Mast ${ }^{9}$, R. M. González \\ Delgado $^{2}$, J.C. Muñoz-Mateos ${ }^{10}$, J. Bland-Hawthorn ${ }^{11}$, D. J. Bomans ${ }^{12}$, A. del Olmo ${ }^{2}$, L. Galbany ${ }^{13,14}$, J.M. Gomes ${ }^{15}$, \\ C. Kehrig ${ }^{2}$, Á. R. López-Sánchez ${ }^{16,17}$, M. A. Mendoza ${ }^{2}$, A. Monreal-Ibero ${ }^{18}$, M. Pérez-Torres ${ }^{2,19}$, \\ P. Sánchez-Blázquez ${ }^{20}$, J. M. Vilchez ${ }^{2}$, and the CALIFA collaboration
}

(Affiliations can be found after the references)

Received 4 March 2015 / Accepted 13 July 2015

\begin{abstract}
Context. The star formation rate (SFR) is one of the main parameters used to analyze the evolution of galaxies through time. The need for recovering the light reprocessed by dust commonly requires the use of low spatial resolution far-infrared data. Recombination line luminosities provide an alternative, although uncertain dust-extinction corrections based on narrowband imaging or long-slit spectroscopy have traditionally posed a limit to their applicability. Integral field spectroscopy (IFS) is clearly the way to overcome this kind of limitation.

Aims. We obtain integrated $\mathrm{H} \alpha$, ultraviolet (UV) and infrared (IR)-based SFR measurements for 272 galaxies from the CALIFA survey at $0.005<$ $z<0.03$ using single-band and hybrid tracers. We aim to determine whether the extinction-corrected $\mathrm{H} \alpha$ luminosities provide a good measure of the SFR and to shed light on the origin of the discrepancies between tracers. Updated calibrations referred to H $\alpha$ are provided. The well-defined selection criteria and large statistics allow us to carry out this analysis globally and split by properties, including stellar mass and morphological type.

Methods. We derive integrated, extinction-corrected $\mathrm{H} \alpha$ fluxes from CALIFA, UV surface and asymptotic photometry from GALEX and integrated WISE $22 \mu \mathrm{m}$ and IRAS fluxes.

Results. We find that the extinction-corrected $\mathrm{H} \alpha$ luminosity agrees with the hybrid updated SFR estimators based on either UV or H $\alpha$ plus IR luminosity over the full range of SFRs $\left(0.03-20 M_{\odot} \mathrm{yr}^{-1}\right)$. The coefficient that weights the amount of energy produced by newly-born stars that is reprocessed by dust on the hybrid tracers, $a_{\mathrm{IR}}$, shows a large dispersion. However, this coefficient does not became increasingly small at high attenuations, as expected if significant highly-obscured $\mathrm{H} \alpha$ emission were missed, i.e., after a Balmer decrement-based attenuation correction is applied. Lenticulars, early-type spirals, and type-2 AGN host galaxies show smaller coefficients because of the contribution of optical photons and AGN to dust heating.

Conclusions. In the local Universe, the $\mathrm{H} \alpha$ luminosity derived from IFS observations can be used to measure SFR, at least in statisticallysignificant, optically-selected galaxy samples, once stellar continuum absorption and dust attenuation effects are accounted for. The analysis of the SFR calibrations by galaxies properties could potentially be used by other works to study the impact of different selection criteria in the SFR values derived, and to disentangle selection effects from other physically motivated differences, such as environmental or evolutionary effects.
\end{abstract}

Key words. galaxies: star formation - galaxies: spiral - galaxies: evolution - techniques: photometric - techniques: spectroscopic

\section{Introduction}

The measurement of the star formation rate (SFR) is crucial for understanding the birth and evolution of the galaxies (Kennicutt 1998a) as it provides information on the amount of gas in galaxies and the efficiency in the formation of stars inside them, which depends strongly on the conditions of the interstellar medium in which they are formed (Kennicutt \& Evans 2012, and references therein). The SFR is, together with galaxy mass, one of the most important parameters that define galaxies and their evolution across cosmic times (Somerville \& Davé 2015; Madau \& Dickinson 2014). Several authors have tried to quantify the rate of ongoing star formation and its evolution with redshift (e.g., Madau et al. 1996; Lilly et al. 1996; Pérez-González et al. 2008; Bouwens et al. 2007, 2011, 2015) using different tracers. These works have shown that the SFR density has declined by roughly

* Table 1 is available in electronic form at http://www . aanda.org a factor of six from $z=2$ to present day (Hopkins \& Beacom 2006).

Until now, the study of the evolution of the SFR has focused on the analysis of the integrated SFR in galaxies, with little attention being paid to where in galaxies (nuclei, bulges, disks) SFR takes places and how the SFR in each of these components evolves separately with redshift. It is remarkable that the use of NIR integral field spectroscopy on $8-10 \mathrm{~m}$ class telescopes is now allowing us to measure the SFR in these different components in distant galaxies, up to $z=1-3$ (e.g., Genzel et al. 2008; Förster Schreiber et al. 2009, 2011a,b; Nelson et al. 2012, 2013; Wuyts et al. 2013; Lang et al. 2014), while the local benchmark for these and possible future studies is still missing except for a few studies rather limited in number and completeness (e.g., Pérez-González et al. 2006; Kennicutt et al. 2007; Leroy et al. 2008, 2012; Bigiel et al. 2008; Blanc et al. 2009; Schruba et al. 2011). In this regard, a correct determination of the calibrators we use to calculate the spatially-resolved SFR is 
essential to compare how the star formation of these different spatial components behave at different wavelength ranges and/or redshifts.

Although SFR calibrators have existed for almost 30 years, the last decade has been particularly fruitful thanks to the multiwavelength surveys of nearby and distant galaxies. The development of the integral field spectroscopy (IFS) technique has allowed us to combine the advantages of both imaging and spectroscopy at optical and near-infrared (NIR) wavelengths. In this paper, we make use of a large and well-characterized sample of nearby galaxies from the Calar Alto Legacy Integral Field Area (CALIFA) survey (Sánchez et al. 2012), which spans the entire color-magnitude diagram to address this fundamental issue. The use of CALIFA allows us to properly determine the $\mathrm{H} \alpha$ and $\mathrm{H} \beta$ fluxes using IFS spectroscopic data. This is particularly important in the case of galaxies with low equivalent widths in emission, especially in $\mathrm{H} \beta$, like many of the objects in the CALIFA sample and in the local Universe in general (Gallego et al. 1995; Brinchmann et al. 2004), where narrowband imaging is not feasible. Furthermore, using these data we can separate the $\mathrm{H} \alpha$ and [NII] flux, while imaging is only feasible in the narrowband if a $[\mathrm{NII}] / \mathrm{H} \alpha$ ratio is assumed. The use of the unique IFS data allows us to obtain precise Balmer-decrement measurements to compute $\mathrm{H} \alpha$ extinction-corrected luminosities. Various studies have shown the importance of computing the extinction using IFS data in nearby regions where the line ratios obtained from the integrated spectra are dominated by regions of lower surface brightness rather than by the brighter regions (Pellegrini et al. 2010; Relaño et al. 2010; Monreal-Ibero et al. 2011). Other advantages related to the IFS data is that we can cover the whole galaxy avoiding problems associated with the limited spatial coverage of long-slit spectroscopy. From these Balmer-corrected $\mathrm{H} \alpha$ luminosities, we compute their corresponding SFRs, which we use as a fiducial measure of the current SFR. However, it is critical to first determine that at least in a statistically sense, no significant fraction of the SFR is being missed when using the extinction-corrected $\mathrm{H} \alpha$ luminosity as SFR estimator. This requires of a combined analysis of this estimator with other SFR estimators, including the continuum ultraviolet (UV) emission, recombination lines of hydrogen and other atomic species together with other estimators less affected by dust attenuation, such as total infrared (TIR) luminosity, monochromatic infrared (IR) emission, or radio emission. The combination of different SFR estimators is also needed to evaluate the potential differences between the current-day SFR given by $\mathrm{H} \alpha$ and that given by tracers sensitive to intermediate-aged stellar population (Kennicutt \& Evans 2012; Calzetti 2013). We order the SFR estimators from less to more sensitive to these populations as $22 \mu \mathrm{m}$, FUV, NUV, TIR. Whether $22 \mu \mathrm{m}$ should precede FUV in this list is still controversial although some results indicate that should be the case (Pérez-González et al. 2006; Alonso-Herrero et al. 2006b; Calzetti et al. 2007, 2010; Kennicutt et al. 2009).

The SFR indicators we consider here come in two types: single-band and hybrid recipes (see Kennicutt \& Evans 2012, Calzetti 2013, for a recent compilation). In the case of the recipes based on a single photometric band, we have used the extinctioncorrected UV (with a extinction correction based on the UV slope; Treyer et al. (2007), Cortese et al. (2008), Muñoz-Mateos et al. (2009); a more precise dust-extinction correction is implicit to the use of UV+IR hybrid tracer), the extinction-corrected $\mathrm{H} \alpha$ and the observed mid-infrared (MIR) or TIR luminosities. The hybrid recipes combine luminosities measured directly (observed UV or $\mathrm{H} \alpha$ ) with that of the light re-emitted by dust after being heated by young massive stars (in our case the MIR or
TIR luminosities), assuming an approximate energy-balance approach (see Gordon et al. 2000, Inoue et al. 2001, Hirashita et al. 2003, Iglesias-Páramo et al. 2006, Calzetti et al. 2007, Kennicutt et al. 2007, 2009, Hao et al. 2011, for more details).

We derive integrated, extinction-corrected $\mathrm{H} \alpha$-based SFRs from the analysis of CALIFA IFS data and compare them with measurements from other SFR tracers. We provide new single-band and hybrid updated SFRs tracers (with and without type-2 AGN being considered) using our integrated, extinctioncorrected $\mathrm{H} \alpha$ SFR as a reference, thanks to the quality of our attenuation correction via Balmer decrement. We pay special attention on the hybrids recipes, providing for the first time, a set of hybrid calibrations for different morphological types and stellar masses. We also analyze the dependence with the color (SDSS $g-r$ ), axial ratio, and ionized-gas attenuation. This analysis is the starting point for a series of papers in which we will study how the SFR in the local Universe is distributed across galaxy components (bulge, disks, nuclei) and bidimensionally. Ultimately, we are interested in knowing how the local SFR density is spatially distributed over galaxies and how these results would compare to similar future studies at high redshift.

This paper is organized as follows: in Sect. 2 we describe the reference sample used in this article, in Sect. 3 we describe the data and the analysis applied to the data, in Sect. 4 we discuss our results, and finally, in Sect. 5 we summarize the main conclusions. Throughout this paper we use a cosmology defined by $H_{0}=70 \mathrm{~km} \mathrm{~s}^{-1} \mathrm{Mpc}^{-1}, \Omega_{\Lambda}=0.7$ and a flat Universe.

\section{The sample}

The galaxies we studied are part of the CALIFA survey (Sánchez et al. 2012). The CALIFA mother sample includes 939 galaxies of all types. A total of $\sim 600$ galaxies will be observed as part of CALIFA, using the Potsdam Multi-Aperture Spectrophotometer (PMAS, Roth et al. 2005) in the PPak mode (Kelz et al. 2006) mounted at the Calar Alto $3.5 \mathrm{~m}$ telescope. The survey is described in detail in the presentation article (Sánchez et al. 2012). As a summary, the CALIFA mother sample (Walcher et al. 2014) includes all galaxies in the DR7 SDSS photometric catalog (Abazajian et al. 2009) with declinations above $7^{\circ}$, spectroscopic redshifts (from the SDSS spectroscopic catalog or elsewhere) in the range $0.005<z<0.03$ and SDSS $r$-band diameters in the range $45^{\prime \prime}<$ D25 $<80^{\prime \prime}$, where D25 refers to the isophote major axis at 25 magnitudes per square arcsecond in the SDSS $r$-band. The observations cover the optical wavelength range 3700-7000 $\AA$, including the most relevant optical emission lines, such as the [OII] $\lambda \lambda 3726,3729 \AA$ doublet, $\mathrm{H} \alpha$ or the [NII] $\lambda \lambda 6549,6583 \AA$ and [SII] $\lambda \lambda 6717,6731 \AA$ doublets. The mother sample is representative of the general galaxy population with the following limits: -19.0 and -23.1 in $r$-band absolute magnitude, 1.7 and $11.5 \mathrm{kpc}$ in half-light radius, and 9.7 and 11.4 in $\log \left(M_{\star} / M_{\odot}\right)$ (Walcher et al. 2014).

This paper makes use of all 380 CALIFA galaxies that have been observed and processed up to Oct 27th 2013, including all those released as part of the Data Release 1 (DR1, see Husemann et al. 2013) and Data Release 2 (DR2, see García-Benito et al. 2015). We refer to this as our reference sample even though some objects do not show detectable line emissions and are not used to derive the $\mathrm{H} \alpha$-based SFR measurements. As this is a random subselection of the mother sample based only on visibility along the observing period, this should be representative in terms of galaxy properties of the entire CALIFA mother sample. To prove this statement we compare the whole mother sample (white areas in Fig. 1) and the galaxies involved in this study (gray-filled 

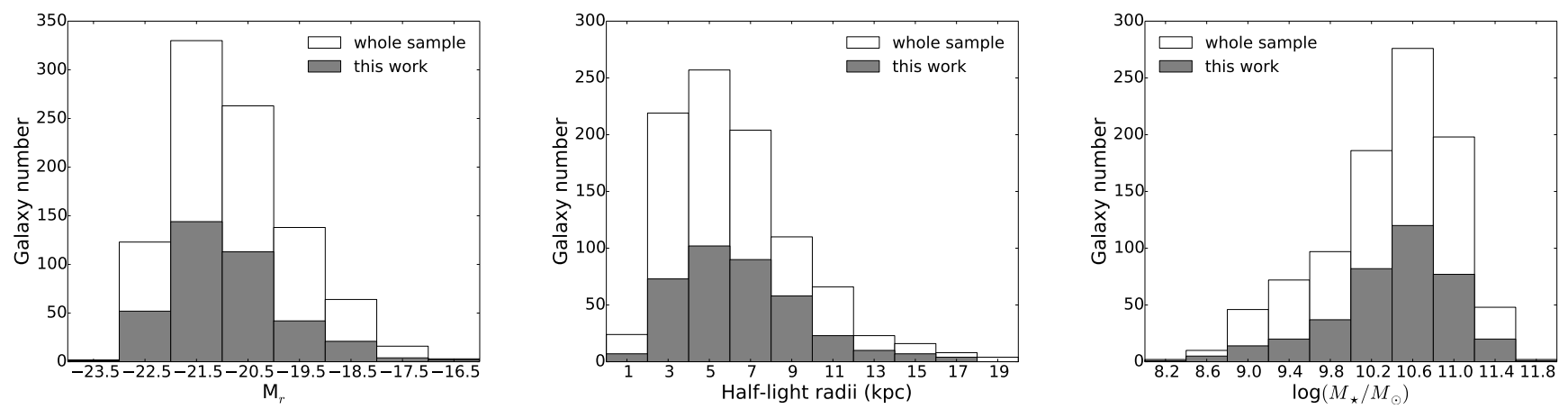

Fig. 1. From left to right: distributions of the $r$-band absolute magnitude, half-light radius, and $\log \left(M_{\star} / M_{\odot}\right)$. The white histograms correspond to the complete CALIFA sample (939 galaxies), and the gray-filled areas correspond to our sample (380 galaxies). A visual inspection of these histograms together with the perform of the K-S test probability show that our sample is representative in terms of galaxy properties of the entire CALIFA sample.

areas). We use a K-S test to check whether the two data samples come from the same distribution. The $\mathrm{K}-\mathrm{S}$ test probability is computed using the limits where the mother sample is representative of the general galaxy population as mentioned before. The values of the probabilities found by the $\mathrm{K}-\mathrm{S}$ test are $40.25 \%$ in half-light radius, $70.95 \%$ in $r$-band absolute magnitude and, finally, $75.55 \%$ in $\log \left(M_{\star} / M_{\odot}\right)$. From these values and from the visual inspection in Fig. 1, we conclude that the subsample we are using is representative of the mother sample, except for a marginal deficiency of intermediate luminosity objects in the range $M_{r}=(-20.5,-21.2)$, which might explain the low $\mathrm{K}-\mathrm{S}$ values but certainly does not bias the results against these systems.

\section{Data and analysis}

\subsection{CALIFA integral field spectroscopy}

\subsubsection{CALIFA survey}

The CALIFA spectra cover the range $3650-7500 \AA$ in two overlapping setups, one in the red (3745-7500 $\mathrm{A})$ at a spectral resolution of $R \sim 850$ (V500 setup) and one in the blue $(3650-4840 \AA)$ at $R \sim 1650$ (V1200 setup), where the resolutions quoted are those at the overlapping wavelength range $(\lambda \sim 4500 \AA)$. For the purpose of deriving extinction-corrected $\mathrm{H} \alpha$ luminosities, we make use of the V500 setup as we are interested in having both $\mathrm{H} \beta \lambda 4861 \AA$ and $\mathrm{H} \alpha \lambda 6563 \AA$ emission lines in the same observing range. The spectral resolution ( $F W H M \sim 6 \AA)$ is sufficient to deblend the $\mathrm{H} \alpha$ emission line from the nearest $[\mathrm{NII}] \lambda \lambda 6548,6584 \AA$ doublet lines. We are using the $\mathrm{v} 1.3 \mathrm{c}$ data products, which yield the measured flux densities corrected for Galactic extinction. The data reduction is explained in detail in Sánchez et al. (2012) and Husemann et al. (2013).

\subsubsection{Aperture spectrophotometry}

For each galaxy for which the CALIFA V500 observations reached the full depth planned $(3 \times 900 \mathrm{~s}$ exposures in a three-point dithered scheme), we generate an integrated spectrum within the largest common aperture possible between the CALIFA and the other complementary data (UV, IR). This aperture has an elliptical shape with a major axis radius of 36 arcsec and the corresponding ellipticity of the galaxy, as given by the minor-to-major axis ratio listed in $\mathrm{NED}^{1}$ for each object. The previous values and the position angle (PA) are measured at the $25.0 \mathrm{mag} / \operatorname{arcsec}^{2}$ isophote at $B$-band provided by the RC 3 catalog (de Vaucouleurs et al. 1991). When this information is not available, we use the SDSS $g$ or $r$-band isophotal photometry. As the extracted aperture is significantly larger $(\sim 4000 \times)$ than the CALIFA pixel size $\left(1 \operatorname{arcsec}^{2}\right)$, effects associated with the treatment of fractional pixels are negligible.

\subsubsection{Continuum subtraction and line-flux measurements}

To minimize systematics associated with the stellar continuum subtraction at low-S/N regimes, we decided to first spatially integrate the datacube within these apertures. This is a particularly interesting use of the IFS data that facilitates both covering the whole galaxy and having a high-S/N in the integrated spectrum. Then, we carry out the necessary corrections to derive total extinction-corrected $\mathrm{H} \alpha$ luminosities. The use of the $\mathrm{H} \alpha / \mathrm{H} \beta$ ratio derived from the integrated spectra is justified instead of correcting for extinction spaxel to spaxel and then coadding the flux to minimize systematics when adding up signals from very noisy individual spaxels, as shown below. Thus, while Sánchez et al. (2011) and Marino et al. (2012) show that the dust attenuation from individual spaxels is a little larger than that derived from the integrated spectra $(1.24 / 1.04$ and $1.19 / 1.03$, respectively), Castillo-Morales et al. (2011) obtain rather similar values in each case. Nevertheless, the interesting point here is how the luminosity-weighted attenuation compares when using individual spaxels with that from the integrated spectra. This question is more relevant as we are analyzing attenuation-corrected $\mathrm{H} \alpha$ luminosities rather than attenuations themselves. For this matter, we select the galaxy NGC 5668 in Marino et al. (2012) as it is a nearby spiral galaxy similar to those used in this work. We find that the difference between computing the average luminosityweighted attenuation from individual spaxels and that derived from the integrated spectrum is less than $1 \%$. From this result, we conclude that we can safely use the $\mathrm{H} \alpha / \mathrm{H} \beta$ ratio derived from the integrated spectra to correct the $\mathrm{H} \alpha$ flux in each galaxy. Besides, this way of obtaining the extinction-corrected $\mathrm{H} \alpha$ luminosity would actually mimic what one could measure in more distant systems for which this work is intended to provide a local benchmark.

The first of those corrections to be applied to our data is to carefully remove the stellar continuum underlying the $\mathrm{H} \beta$ and

https://ned.ipac.caltech.edu 
$\mathrm{H} \alpha$ lines. This is done by means of adjusting a linear combination of two single stellar population (SSP) evolutionary synthesis models of Vazdekis et al. (2010) based on the MILES stellar library (Sánchez-Blázquez et al. 2006) to the spectrum obtained for each aperture. Two set of models with a Kroupa IMF (Kroupa 2001) are combined. One set contains models (considered as a young stellar population) with ages of $0.10,0.50$, and $0.79 \mathrm{Gyr}$. A second set (considered as an old stellar population) involves ages of 2.00, 6.31, and 14.13 Gyr. For each age, we considered five different metallicities with $[\mathrm{M} / \mathrm{H}]$ values equal to $0.00,0.20$, $-0.40,-0.71$, and -1.31 dex offset from the solar value.

Different wavelength ranges corresponding to the emission lines from the ionized gas and sky-lines are masked and not included in the fit. The basic steps applied in this method are the following: (1) shift the SSP templates to match the systemic velocity of the integrated spectrum; (2) convolve each stellar population model with a Gaussian profile so the absorption features could be broadened to match those of the integrated spectrum; (3) redden the spectrum using a $k(\lambda)=R_{V}(\lambda / 5500 \AA)^{-0.7}$ power law, where $R_{V}=5.9$, as given by Charlot \& Fall (2000); and (4) finally, the best linear combination of SSPs is determined by a $\chi^{2}$ minimization.

Once we obtain the best underlying continuum of the stellar population, we subtract it from the original integrated spectrum to derive the pure emission line spectrum. The emission line fluxes are computed from this residual spectrum. As some residual continuum could still be present in some cases, we do not simply add all the flux in fixed windows in wavelength. Instead, we compute the $\mathrm{H} \beta$ and $\mathrm{H} \alpha$ emission line fluxes by fitting Gaussian functions plus a low-order polynomial function. Figure 2 shows the original integrated spectrum for three galaxies with different levels of emission-line strength, IC 4215 , NGC 2906, and NGC 5630 in black. The best fit to the spectrum of the underlying stellar population is shown in red and the emission-line spectrum produced by the ionized gas is shown in blue. Gray-colored wavelength ranges correspond to the emission lines and sky lines masked out in the fitting procedure.

A proper estimation of the $\mathrm{H} \beta$ emission line flux is crucial to obtain a reliable Balmer decrement and, from it, the correction for extinction of the $\mathrm{H} \alpha$-based SFR. The method applied here is expected to be a robust procedure as long as a relatively wide wavelength coverage is available (see Mármol-Queraltó et al. 2011) and the models contain an extensive range of ages and metallicities. When the whole spectral range (3750-7000) $\AA$ is used for the stellar continuum fitting, however, we still detect systematic residuals around the $\mathrm{H} \beta$ absorption line. The treatment of these spectral features is particularly critical. They could be real because of the limitation of the models in reproducing simultaneously a broad wavelength range and the $\mathrm{H} \beta$ region, or introduced during the data reduction. We have also checked that adding an intermediate age population in the linear combination of the SSPs does not change the overall results. For that reason, the stellar continuum fitting around the $\mathrm{H} \beta$ line is done for other wavelength ranges using the method explained before. The new spectral ranges used are (3700-5500) $\mathrm{A},(4100-5500) \AA$, and (4800-5500) $\AA$. Given that the residual continuum around $\mathrm{H} \alpha$ and $\mathrm{H} \gamma$ emission lines does not show systematic uncertainties, we determine the $\mathrm{H} \beta$ flux by anchoring to $\mathrm{H} \alpha$ and $\mathrm{H} \gamma$ fluxes based on theoretical line ratios and extinction coefficients. In high $\mathrm{S} / \mathrm{N}$ spectra, this $\mathrm{H} \beta$ emission line flux estimation is compared with the values obtained when different spectral ranges for the stellar continuum fitting are used. Finally, we obtain that the $\mathrm{H} \beta$ emission fluxes calculated using the spectral range
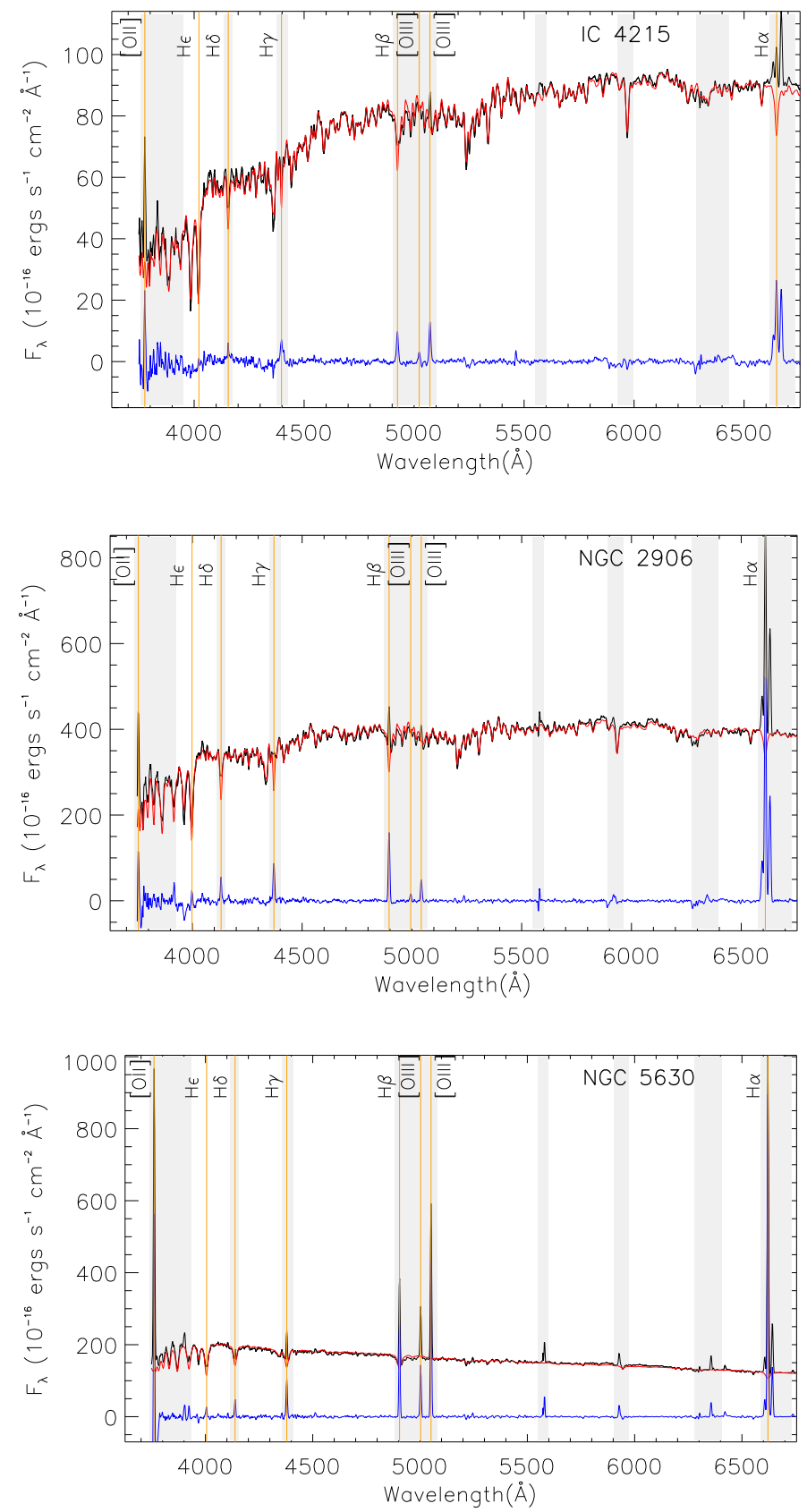

Fig. 2. Top panel: original integrated spectrum for galaxy IC 4215 is shown in black, the best fit to the stellar population appears in red (using the 3745-7500 $\AA$ entire spectral range), and pure emission line spectrum (after the subtraction of a residual continuum shape applying a smooth function). This galaxy shows a small equivalent width in the $\mathrm{H} \beta$ line. Center panel: same as top panel for the galaxy NGC 2906. Note that in this case the spectrum shows more prominent $\mathrm{H} \alpha$ and $\mathrm{H} \beta$ emission lines. Bottom panel: in this case, NGC 5630 shows a typical emission line dominated spectrum with very high EWs of $\mathrm{H} \alpha$ and $\mathrm{H} \beta$ emission lines. These spectra show the variety in levels of emission line strength in our galaxies. A proper subtraction of the underlying stellar population is required to obtain the estimation of the emission line fluxes.

(4800-5500) $\AA$ are in best agreement with the theoretical emission fluxes.

We impose a minimum $\mathrm{S} / \mathrm{N}$ for both $\mathrm{H} \alpha$ and $\mathrm{H} \beta$ emission lines fluxes to obtain a precise measurement of the extinction using the Balmer decrement. The $\mathrm{S} / \mathrm{N}$ emission line estimation is done using a formal method calculating the ratio 
between the Gaussian amplitude at $\mathrm{H} \beta$ and the root mean square in the near featureless continuum. A visual inspection of the continuum-subtracted spectra at $\mathrm{H} \beta$ is performed for all the analyzed galaxies and a minimum $S / N>5$ is considered for $\mathrm{H} \beta$ emission line detection. The number of galaxies with detected $\mathrm{H} \beta$ emission is 272 over the initial 380 galaxies. This is the sample (listed in Table 1 for reference) that will be used in the rest of the analysis.

The spectrophotometric accuracy in CALIFA DR1 was checked using SDSS $g$ and $r$-band photometry, which are both entirely covered by the V500 setup. Husemann et al. (2013) found a systematic offset of $\Delta(g-r)=-0.06$ mag (median) with a scatter of only 0.05 mag. This means that the spectrophotometric accuracy across most of the covered wavelength range is $6 \%$ for the CALIFA data. This value is included in our error estimation for the emission line fluxes.

\subsubsection{Flux corrections and uncertainties}

We also apply some corrections, such as aperture corrections and those associated with the spatial masking of field stars and background galaxies applied to the datacubes, to our data. We use aperture corrections for the galaxies whose line emission is expected to be more extended that the CALIFA field of view (FOV). Our main criterion is to select the band that would first trace the SFR, and second, that it would do it with the best spatial resolution possible. Also, we want to have them available for most galaxies in our sample. Here, we assume that the $\mathrm{H} \alpha$ light distribution beyond the FOV is similar to that of the ultraviolet light in each galaxy individually. Besides, the UV band has the highest spatial resolution within the bands we are using that are closely related to the SFR. Thus, for that purpose we use the GALEX NUV images (deeper and available for a few more objects than the FUV images). We compute the difference between the NUV magnitudes obtained for the 36-arcsec-aperture and the asymptotic magnitudes. We fit the aperture correction data for the whole mother sample (those galaxies having NUV data) as a function of the galaxy size, given by the $i s o A$ in the $r$-band from the SDSS. The correlation between aperture correction and isophotal diameter is the strongest of all those analyzed and it will be used for galaxies without NUV magnitudes. The resulting median correction is around 1.4. The observed $\mathrm{H} \alpha$ luminosities already corrected for aperture effects are listed in Table 1.

The spatial masking is applied over the datacubes before performing the stellar continuum fitting. That means that the light from spaxels contaminated by field stars and background objects is not summed up at this stage. Then, we correct the emissionline fluxes for the flux coming from those missing spaxels. The mean value for the correction factor is $2.2 \%$. The corrections are only applied over $44.7 \%$ of the galaxies, i.e., those that have contaminating sources. These correction factors are obtained comparing the aperture fluxes between two sets of synthetic continuum-subtracted narrowband images. One of them without the flux from the corresponding contaminated pixels and the other where the flux from those pixels is obtained by local interpolation.

Once $\mathrm{H} \alpha$ and $\mathrm{H} \beta$ emission line fluxes are computed, we correct the $\mathrm{H} \alpha$ flux for dust attenuation assuming that the relation between $\mathrm{H} \alpha$ reddening and extinction follows the foreground dust screen approximation. Although this could be a possible source of systematic error in the analysis, some models have shown that when applied to normal star-forming galaxies the dust geometry assumed does not introduce significant systematic

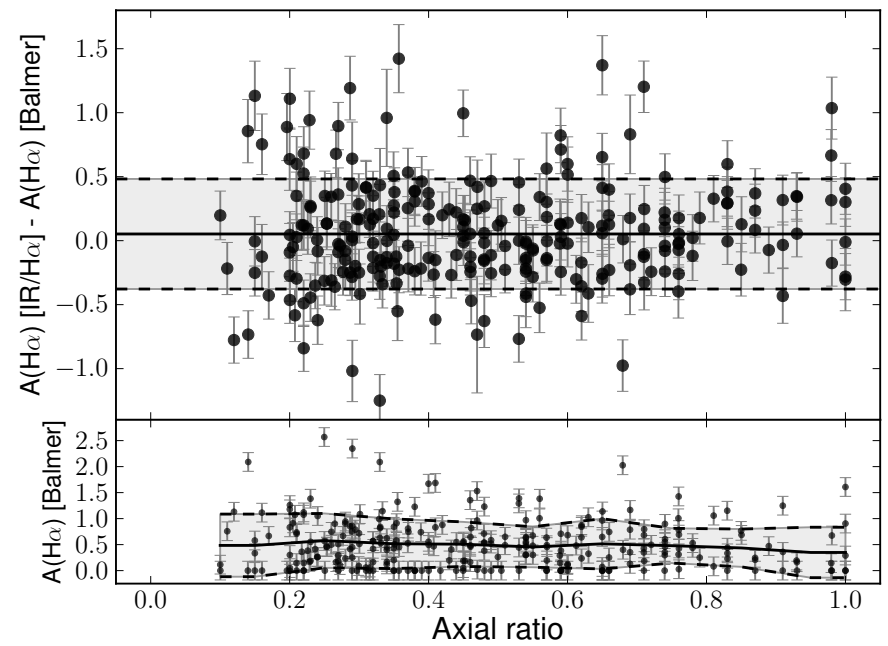

Fig. 3. Top panel: difference between $\mathrm{H} \alpha$ attenuations derived from the ratio of $\mathrm{IR} / \mathrm{H} \alpha$ and those obtained using the Balmer decrement as a function of galaxy axial ratio. Solid and dashed black lines correspond to the mean and $1 \sigma$ values, $(0.05 \pm 0.43)$ mag, after applying a rejection of $4 \sigma$. Because of the good agreement between both attenuations computed from different methods, we can safely assume that our Balmer decrement attenuations yield proper results. Besides, we do not find any systematic residuals against the axial ratio parameter associated with highly-inclined galaxies. Bottom panel: $A(\mathrm{H} \alpha)$ derived from the Balmer decrement as a function of the axial ratio (used as a proxy for inclination). Gray shadow corresponds to the $1 \sigma$ intervals around the mean value showed in black solid line.

errors (Jonsson et al. 2010). See a detail discussion about the use of attenuation corrections based on Balmer decrements with a Galactic extinction curve and a foreground screen dust geometry in Sects. 3.3 and 6.3 in Kennicutt et al. (2009). For the attenuation correction, we use an intrinsic Balmer ratio of 2.86 for case B recombination (Osterbrock 1989) at electron temperature $T_{\mathrm{e}}=10000 \mathrm{~K}$ and density $n_{\mathrm{e}}=100 \mathrm{~cm}^{-3}$ (Hummer \& Storey 1987) using the following expression 1 , where $K_{\mathrm{H} \alpha}=2.53$ and $K_{\mathrm{H} \beta}=3.61$ are the extinction coefficients for the Galactic extinction curve from Cardelli et al. (1989):

$A(\mathrm{H} \alpha)=\frac{K_{\mathrm{H} \alpha}}{-0.4 \times\left(K_{\mathrm{H} \alpha}-K_{\mathrm{H} \beta}\right)} \times \log _{10} \frac{F_{\mathrm{H} \alpha} / F_{\mathrm{H} \beta}}{2.86}$.

As an example of how little this attenuation correction would vary among extinction curves and dust-to-stars geometries, we compare the ratio between the $A(\mathrm{H} \alpha)$ attenuations for the same $A(\mathrm{H} \beta)$ using the Cardelli et al. (1989) $\left(R_{V}=3.1\right)$ law above and the Calzetti et al. (2000) $\left(R_{V}=4.05\right)$ attenuation law. We obtain $A(\mathrm{H} \alpha)_{\text {Calzetti }} / A(\mathrm{H} \alpha)_{\text {Cardelli }}=1.03$.

Note that the standard $\mathrm{H} \alpha / \mathrm{H} \beta$ ratio used in Eq. (1) is only valid for the particular ionization conditions indicated above, but values below 2.86 are also physically possible in HII regions, depending on the electron density, effective temperature, and, therefore, on the chemical abundance. This leads to a number of galaxies for which we assumed $A(\mathrm{H} \alpha)=0$. The computed extinction values $A(\mathrm{H} \alpha)$ are listed in Table 1 . The $\mathrm{H} \alpha$ luminosity corrected by attenuation and by the effects mentioned along this section is referred to hereafter as $\mathrm{H} \alpha_{\text {corr }}$.

We test whether the foreground dust screen approximation has an effect on edge-on galaxies. For that purpose, we plot the difference between $A(\mathrm{H} \alpha)$ derived from the Balmer decrement and the $A(\mathrm{H} \alpha)$ values derived from the ratio of $\mathrm{IR} / \mathrm{H} \alpha$ as a function of galaxy axial ratio (see top panel in Fig. 3). 
The expression used to derive the attenuation from the ratio of $\mathrm{IR} / \mathrm{H} \alpha$ is $A(\mathrm{H} \alpha)=2.5 \times \log \left[1+a_{\mathrm{IR}} \times L(\mathrm{IR}) / L\left(\mathrm{H} \alpha_{\text {obs }}\right)\right]$ (see Kennicutt et al. 2009, Eq. (2)). In this case, L(IR) corresponds to $L(22 \mu \mathrm{m})$ available for a larger number of galaxies in our sample than $L(T I R)$. The coefficient $a_{\mathrm{IR}}$ is equal to $0.015_{-0.006}^{+0.018}$ (average value derived from our sample in Sect. 4.5.2). The value given by Kennicutt et al. (2009) is $a_{\mathrm{IR}}=$ $0.020 \pm 0.001 r \pm 0.005 \mathrm{~s}$, which is in good agreement with ours even taking into account that they are obtained from different samples. Finally, the difference between both $A(\mathrm{H} \alpha)$ estimations yields mean and $1 \sigma$ values of $(0.05 \pm 0.43)$ mag after doing a rejection of $4 \sigma$. This value shows that both methods produce compatible results. As we do not see systematic residuals against the axial ratio parameter, we conclude that we do not find a different behavior in the case of highly-inclined galaxies.

Finally, the uncertainty in the $\mathrm{H} \alpha$ flux is estimated from a random redistribution of the residuals obtained after fitting a Gaussian function to the pure emission-line spectrum in the spectral range around $\mathrm{H} \alpha$ emission. The new residual spectrum is added to the pure emission-line spectrum and a new Gaussian fit is performed. This procedure is repeated 1000 times and the standard deviation of the computed $\mathrm{H} \alpha$ fluxes is considered as the error in the $\mathrm{H} \alpha$ flux. On the other hand, the comparison between the measured $\mathrm{H} \beta$ line fluxes and those expected from the $\mathrm{H} \alpha / \mathrm{H} \gamma$ Balmer decrements for the same ionized-gas physical conditions gives us an estimation of the $\mathrm{H} \beta$ flux uncertainty. A dispersion of $\sigma=7 \%$ centered around unity is obtained across the whole sample. This method provides much larger uncertainties compared with the method using the redistribution of the residuals around the $\mathrm{H} \beta$ emission line. The reported error includes the potential uncertainties in the modeling of the stellar continuum and it is taken as a conservative upper limit for the error in the $\mathrm{H} \beta$ flux. This $\mathrm{H} \beta$ flux uncertainty propagates to a much larger one in the corrected $\mathrm{H} \alpha$ flux. A standard error propagation method is used to compute the uncertainties in other quantities, such as extinction or luminosity.

\subsection{GALEX UV imaging}

For nearly two thirds of the galaxies in the CALIFA mother sample, we were able to collect UV observations available from the Galaxy Evolution Explorer $\left(G_{A L E X}^{2}\right.$ ) archive (see Martin et al. 2005). In most cases (655 out of the 663 objects with GALEX UV data), this includes both far-ultraviolet (FUV, effective wavelength $\lambda_{\text {eff }} \sim 1516 \AA$ ) and near-ultraviolet (NUV, $\lambda_{\text {eff }} \sim 2267 \AA$ ) bands, 200 of them included in the sample of the 272 galaxies we analyzed.

The GALEX archive provides simultaneous co-aligned FUV and NUV images with a pixel scale of 1.5 arcsec per pixel and a spatial resolution (FWHM) of 4-5 arcsec. We selected galaxies located within the central 0.5 -degree radius of the 1.2-degrees circular GALEX FOV. We also imposed that the whole galaxy is included in the GALEX FOV. To calculate the integrated mean flux of the galaxy, foreground stars and other targets in the field were identified and removed by averaging the interpolation along rows and columns from the GALEX images.

The typical background in the GALEX UV images is very low so the distribution of count rates in each image typically follows a non-Gaussian distribution. Because of this peculiarity, we estimate the background using the mean instead of the median or the mode used at high background levels, such as ground-based optical or NIR imaging (see Gil de Paz et al. 2007). Surface and

2 http://galex.stsci.edu/GalexView/ aperture photometry was then carried out for each galaxy, using the IRAF task ELLIPSE, as described in Gil de Paz et al. (2007), within elliptical isophotes with fixed ellipticity and position angle (the same ones used for the extraction of the spectra from the CALIFA datacubes). In addition to the 36 -arcsec aperture mentioned above we also extracted UV photometry in other concentric elliptical apertures until the error in the surface photometry reached 0.8 mag (including both background-subtraction and photon noise). From each set of concentric elliptical apertures, we finally obtained asymptotic magnitudes for the whole sample (Gil de Paz et al. 2007). These asymptotic magnitudes are those previously applied in Sect. 3.1.4 to obtain the aperture corrections.

As the UV luminosity suffers from severe attenuation by dust this has to be corrected to properly estimate the SFR. The most commonly accepted method to estimate the dust attenuation at UV wavelengths is to use the ratio between the IR $(22-25 \mu \mathrm{m}$ MIR, FIR, or TIR) and the UV flux (also known as infrared excess or simply IRX). This is equivalent to the use of hybrid SFR estimators, which includes information from these two wavelengths and is the approach used in Sect. 4.5.2. The IR/UV ratio is almost independent of the dust properties and the relative distribution of dust and stars (Buat et al. 2005). However, it depends on the age of the dust-heating populations (see Cortese et al. 2008). In the context of this section, we analyzed only the case when no IR data is available. Should that be the case, a relation between the FUV-NUV color and the infrared excess could be used instead (see Muñoz-Mateos et al. 2009, Eq. (2)). For the sake of simplicity, and given the intrinsic large dispersion of the IRX- $\beta$ relationship (see Sect. 4.5.2 for the IRX- $\beta$ relationship in our sample), we make use of the following linear relation between $A(\mathrm{FUV})$ and FUV-NUV and the corresponding $\pm 1 \sigma$ prediction intervals:

$A(\mathrm{FUV})=0.556+2.292 \times(\mathrm{FUV}-\mathrm{NUV})$

This linear empirical relation is based on the analysis of UV and infrared surface photometry of the SINGS sample (Kennicutt et al. 2003) carried out by Muñoz-Mateos et al. (2009). These authors use FUV, NUV, and TIR luminosity profiles with the same spatial resolution to compute both, FUV-NUV colors and $A$ (FUV) attenuations via the $L(\mathrm{TIR}) / L(\mathrm{FUV})$ ratio using the expression given by Buat et al. (2005). This is similar to the IRX- $\beta$ relationship first studied by Meurer et al. (1995) and calibrated for starburst galaxies. However, Muñoz-Mateos et al. (2009) use star-forming galaxies that have lower values of the extinction for a given FUV-NUV color. We emphasize that these relations (based on the UV color alone) should only be used as a rough estimate of the UV light attenuation. These relations have some (limited) statistical meaning, but very little use in a case-by-case basis. More recently, Hao et al. (2011) provided a physical motivation for this linear relationship between UV color and attenuation and yielded a y-intercept of $-0.084 \mathrm{mag}$ (that corresponds to a FUV-NUV color in the absence of dust of $0.022 \pm 0.024$ mag) and a slope of 3.83. Taking into account that the intrinsic FUV-NUV color for zero attenuation is different in both cases (because of the noisy relation between $A(\mathrm{FUV})$ and FUV-NUV color), we decide to use Eq. (2) (J. C. Muñoz-Mateos, priv. comm.) as in this case we have prediction intervals as a function of the UV color.

We apply Eq. (2) to galaxies that have a FUV-NUV color less or equal to $1 \mathrm{mag}$. Galaxies with colors FUV-NUV $>1$ could correspond to either red galaxies with old stellar populations or galaxies with large amounts of dust reddening. In our sample, the mean value of the dust attenuation in the FUV 
C. Catalán-Torrecilla et al.: Star formation in the local Universe from the CALIFA sample. I.

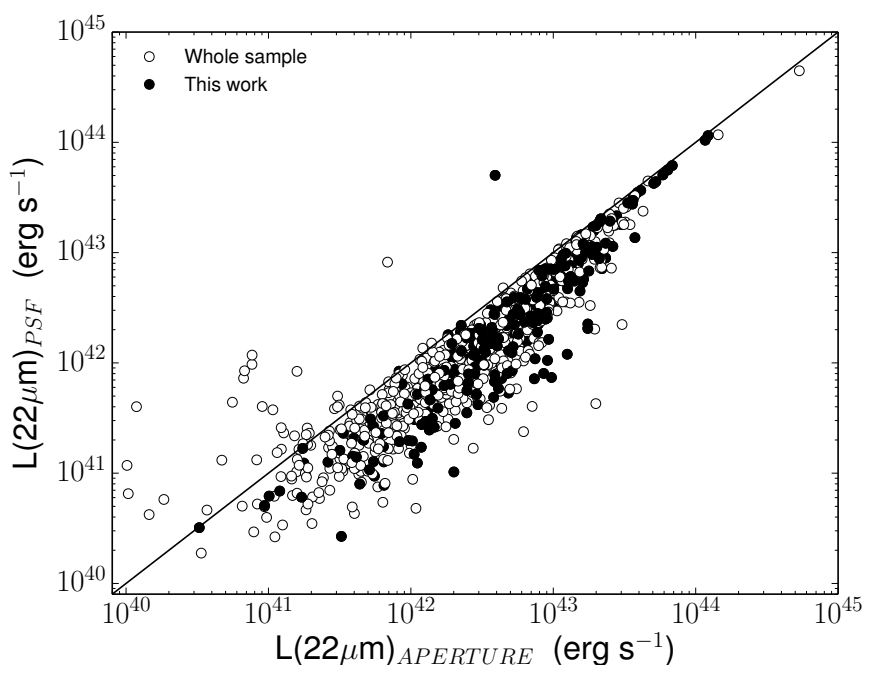

Fig. 4. Comparison between the WISE $22 \mu \mathrm{m}$ PSF and aperture photometry for the entire CALIFA mother sample (white points) and for the galaxies used in this work (black points). Solid line corresponds to the 1:1 line and is given for reference. Although the WISE Source Catalog is ideal for point sources and the resolution of the WISE $22 \mu \mathrm{m}$ band is wide enough $(F W H M \sim 11$ arcsec) it does not seems to be suitable for the CALIFA sample.

is $1.73 \mathrm{mag}$ and vary from 0.81 to $2.80 \mathrm{mag}$, as is found by other authors (e.g., Buat et al. 2005; Burgarella et al. 2005). The FUV-NUV colors, $L(\mathrm{FUV})$, and $L(\mathrm{NUV})$ in $\mathrm{ergs} \mathrm{s}^{-1}$ for 200 galaxies over the 272 galaxies we analyzed are listed in Table 1.

\subsection{WISE MIR imaging}

The Wide-field Infrared Survey Explorer (WISE, Wright et al. 2010) surveyed the entire sky at MIR wavelengths 3.4, 4.6, 12, and $22 \mu \mathrm{m}$ (W1 through W4 bands) with five $\sigma$ point-source sensitivities of $\sim 0.08,0.11,0.8$, and $4 \mathrm{mJy}$, respectively. The WISE All-sky Data Release is available through the Infrared Science Archive (IRSA ${ }^{3}$ ). It includes imaging (Image Atlas) and PSFphotometry source catalogs (Source Catalog) for all four WISE bands for the entire CALIFA mother sample. We make use of the WISE $22 \mu \mathrm{m}$ data (W4-band) for information on the amount of (mainly UV) photons being processed through dust absorption and re-emitted. The WISE Source Catalog is optimized for point sources and, in spite of the resolution of the $22 \mu \mathrm{m}$ data $(F W H M \sim 11$ arcsec) and the size of the CALIFA galaxies (limited in diameter to $\sim 1$ arcmin), this photometry catalog might not be appropriate for our sample (see Fig. 4). Therefore, we decided to perform aperture photometry using the Image Atlas to calculate the integrated $22 \mu \mathrm{m}$ fluxes and magnitudes. We obtain aperture photometry in circular apertures that enclosed the entire flux from the source. A circular annulus around this aperture is used to compute the sky. We derive $L(22 \mu \mathrm{m})$ in $\mathrm{erg} \mathrm{s}^{-1}$ for 265 objects out of the 272 galaxies with detected $\mathrm{H} \beta$ emission included in this work (see Table 1). It has been pointed out by several authors (Wright et al. 2010; Jarrett et al. 2013; Brown et al. 2014) that star-forming galaxies measured with the WISE $22 \mu \mathrm{m}$ filter are systematically brighter by $\sim 10 \%$ than that inferred from Spitzer IRS and $24 \mu \mathrm{m}$ data. This factor has been applied in our $22 \mu \mathrm{m}$ luminosities along the article. Values of $L(22 \mu \mathrm{m})$ in Table 1 should be multiplied by this correction factor.

\footnotetext{
http://irsa.ipac.caltech.edu
}

\subsection{TIR fluxes: WISE, IRAS, and AKARI}

Although the longest WISE band already provides relevant information on the attenuation of the UV light associated with regions of star formation, a significant fraction of the energy reradiated in the IR by dust emerges at longer wavelengths. To account for possible differences in the dust temperature or grainsize distribution, which could hamper the use of WISE $22 \mu \mathrm{m}$ alone, we have also collected IRAS photometry for the entire CALIFA mother sample.

The Infrared Astronomical Satellite (IRAS, Neugebauer et al. 1984) surveyed $96 \%$ of the sky in four wavelength bands at $12,25,60$, and $100 \mu \mathrm{m}$. Our main data source is the IRAS Faint Source Catalog v2.0 (FSC; Moshir et al. 1990) with a detection limit about one magnitude deeper than the Point Source Catalog (PSC; Beichman et al. 1988), reaching a depth of $\sim 0.2 \mathrm{Jy}$ at 12, 25 , and $60 \mu \mathrm{m}$ and greater than $1.0 \mathrm{Jy}$ at $100 \mu \mathrm{m}$. The FSC is at least $98.5 \%$ reliable at 12 and $25 \mu \mathrm{m}$ and $\sim 94 \%$ at $60 \mu \mathrm{m}$.

We performed a cross-match of the CALIFA mother sample with the IRAS Faint Source Catalog (closest IRAS source within 40 arcsec), finding 488 galaxies in common for the four IRAS bands. Within the IRAS FSC catalog, a flux density measurement can either be high quality $(F Q U A L=3)$, moderate quality $($ FQUAL $=2)$, or just an upper limit $(F Q U A L=1)$ (Moshir et al. 1992). We make use of only high and moderate quality measurements available for the CALIFA mother sample galaxies, which yielded $12,25,60$, and $100 \mu \mathrm{m}$ detections for 200, 203, 486, and 443 sources, respectively. Note that poor spatial resolution of IRAS in any of these bands ensures that the flux measurements in the FSC are accurate for the CALIFA objects as long as the object is relatively isolated, but could have an impact on the TIR measurements of galaxies in pairs or close groups.

As noted above, the fraction of galaxies with $25 \mu \mathrm{m}$ measurements is significantly lower than that of galaxies with $60 \mu \mathrm{m}$ and $60+100 \mu \mathrm{m}$ measurements. This is due to the comparable detection limit of IRAS at 25 and $60 \mu \mathrm{m}$ but larger flux densities of nearby star-forming galaxies at these latter wavelengths. To recover a larger fraction of galaxies with total infrared flux densities (TIR, i.e., $8-1000 \mu \mathrm{m}$ ) we decided to combine the WISE $22 \mu \mathrm{m}$ photometry with that from IRAS to determine the galaxies TIR luminosity. The reliability of this procedure is demonstrated by the tight correlation between our WISE $22 \mu \mathrm{m} \mathrm{lu}$ minosities and those detected at $25 \mu \mathrm{m}$ with high and moderate quality flux by IRAS (blue and red points in Fig. 5). Therefore, we can confidently use our WISE $22 \mu$ m photometry to increase the number of CALIFA galaxies with TIR measurements. In addition, we are going to use the WISE $22 \mu \mathrm{m}$ measurements instead of the IRAS $25 \mu \mathrm{m}$ in the corresponding IR SFR tracers, as both are found compatible, and there are significantly more measurements from WISE $22 \mu \mathrm{m}$. Previous studies (Kennicutt et al. 2009; Calzetti et al. 2010) show that the average ratio between $24 \mu \mathrm{m}$ and $25 \mu \mathrm{m}$ luminosities is $0.98 \pm 0.06$. In our case, we find that the average ratio between $25 \mu \mathrm{m}$ and $22 \mu \mathrm{m}$ luminosities is $1.05 \pm 0.22$ when using high quality $25 \mu \mathrm{m}$ IRAS measurements. Note that the galaxies used in our work are more distant and, therefore, the photometric errors tend to be larger.

As we are interested in estimating the TIR luminosity for our sample of galaxies, we add AKARI photometry measurements at 140 and $160 \mu \mathrm{m}$ from the AKARI/FIS All-Sky Survey Bright Source Catalogue (Yamamura et al. 2010). Using a cone search of 90 arcsec, we find 247 galaxies at $140 \mu \mathrm{m}$ and 70 galaxies at $160 \mu \mathrm{m}$ with high quality data, which implies confirmation of the source detection and a reliable flux estimation. By adding 


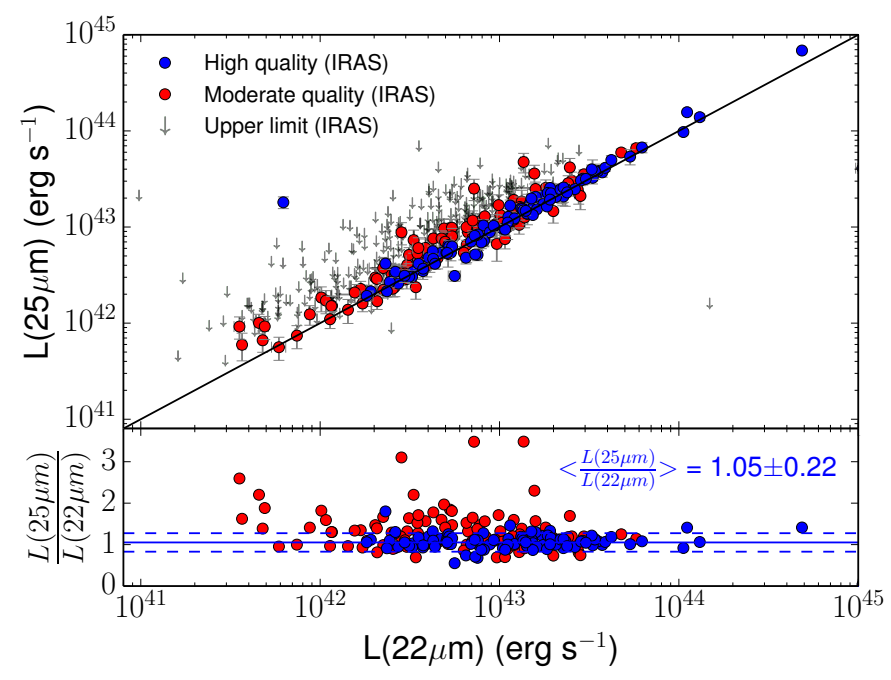

Fig. 5. Comparison between $22 \mu \mathrm{m}$ WISE and $25 \mu \mathrm{m}$ IRAS luminosities for the CALIFA mother sample. Blue and red points correspond to high and moderate quality IRAS $25 \mu \mathrm{m}$ data, respectively. Arrows represent upper limits for the same IRAS band. The solid line shows a linear 1:1 relation for reference. The average ratio between $25 \mu \mathrm{m}$ and $22 \mu \mathrm{m}$ luminosities is $1.05 \pm 0.22$ when using high quality $25 \mu \mathrm{m}$ IRAS measurements. This tight relation allows us to use them interchangeably using the previous conversion factor.

these measurements, we include information at wavelengths at the peak of the spectral energy distribution (SED) and beyond.

We test the consistency of the IRAS and AKARI measurements when possible (IRAS $60 \mu \mathrm{m}$ vs. AKARI $65 \mu \mathrm{m}$ and IRAS $100 \mu \mathrm{m}$ vs. AKARI $90 \mu \mathrm{m})$. In general, AKARI gives lower flux values than expected from the IRAS photometry measurements for those wavelengths in common. Besides, we also find that AKARI 140 and $160 \mu$ m fluxes tend to fall (quite systematically) below the values predicted by our best-fitting infrared SEDs at these wavelengths when data at all IRAS bands is also available. For this reason, we use AKARI 140 and $160 \mu \mathrm{m}$ bands as lower limits to help discriminate between different dust SEDs, which still provides information for the fits in cases where some IRAS fluxes could be missing.

Finally, by fitting a set of IR templates from Chary \& Elbaz (2001), Dale \& Helou (2002) and Rieke et al. (2009) to the WISE $22 \mu \mathrm{m}$, IRAS, and AKARI photometry, we derive TIR fluxes for 547 (out of 939) galaxies in the CALIFA mother sample, 221 of them included in the sample of the 272 galaxies analyzed in this work. The fitting procedure used to derive TIR fluxes is described in detail in Pérez-González et al. (2008). As a brief explanation, the code carries out a template-fitting procedure using the rest-frame effective wavelengths (i.e., $\left.\lambda_{\mathrm{eff}} /(1+z)\right)$. Then, it integrates the best-fit spectra in the wavelength range $(8-1000) \mu \mathrm{m}$ for a total of 100 Monte-Carlo simulated SEDs per galaxy. The average of the TIR individual MC-simulated SED luminosities obtained for each galaxy is listed in Table 1. We adopt these values as the best measure of the TIR luminosity.

\section{Results}

Our aim is to determine the different SFR estimators (singleband and hybrids) for the CALIFA sample and, in particular, ensure that $\mathrm{H} \alpha_{\text {corr }}$ can be safely used for future statistical spatiallyresolved studies, at least in the local Universe.

There are three different ways to carry out this analysis: by comparing fluxes, luminosities (or SFRs), and surface brightnesses (or SFR surface densities). Ideally, we would also like to include in this comparison as many SFR tracers as possible. In the latter case, this implies having good spatial resolution to identify the area in the galaxy responsible for the current activity of star formation. Specifically, in the case of the IR measurements this is usually not possible as the WISE and IRAS data do not provide such a high spatial resolution. For this reason, the analysis of the SFR surface density cannot be carried out in all tracers. Therefore, for most of this section we rely on the comparison between integrated SFR measurements. The use of fluxes for this comparison is excluded as the correlations would be mainly driven by the wide range in distances spanned by our sample $(0.005<z<0.03)$.

However, the linear correlation of the integrated SFR between different tracers, more than the similarity between the SFR tracers, could be partly due to galaxies with different total SFR but similar SFR surface density (scaled-up versions of low-SFR surface density objects). Using the tracers with higher spatial resolution, $\mathrm{UV}$ and $\mathrm{H} \alpha$, (see Sect. 4.1 for a description of the SFR calibrators found in the literature), we compare in Sect. 4.2 the predictions of the SFR surface density.

In the majority of cases, the recipes used to determine the integrated SFR found in the literature are based on samples with ill-defined selection criteria, where the bias toward or against low-metallicity, low-extinction galaxies or highly extinct systems has not been accounted for. We compare their predictions for the CALIFA sample in Sect. 4.3. We describe the possible discrepancies among the different SFR tracers used until now in Sect. 4.4.

Finally, in Sect. 4.5 we provide updated calibrations for the CALIFA sample assuming that (as proven across this section) the $\mathrm{H} \alpha$ extinction-corrected SFR provides a reliable SFR estimator in the local Universe. Thus, we anchor both single-band and hybrid tracers to the $\mathrm{H} \alpha$ extinction-corrected SFR tracer. We also explore the origin of the difference between the SFR tracers used as a function of galaxy properties, such as morphological type, stellar mass, SDSS $g-r$ color, axial ratio, or attenuation.

As we are interested in separating star-forming galaxies from the galaxies hosting an AGN, the plots provided in the following sections show SF galaxies in blue and type-2 AGN host galaxies in orange. The same color-coding is used in the rest of the paper. The information regarding the optical AGN classification can be found in Walcher et al. (2014). Briefly, the authors use the emission-line fluxes for all SDSS spectra of DR7. They create a classical [O III] $\lambda 5007 / \mathrm{H} \beta$ vs. [N II] $\lambda 6583 / \mathrm{H} \alpha$ diagram (Baldwin et al. 1981) to classify the objects and discriminate between different ionization sources at the center of CALIFA galaxies (see Walcher et al. 2014, Fig. 17). For the galaxies with no classification, we extract the same $3^{\prime \prime}$-diameter circular apertures in the nuclear part. Then, we follow the same criteria as described in Walcher et al. (2014) to classify them into their corresponding activity type: either SF or type-2 AGN host galaxies.

\subsection{Recipes for determining the SFR in galaxies}

The SFR indicators considered are of two types: single-band and hybrid. In the case of the recipes based on a single photometric band, we use the extinction-corrected UV (from the UV slope), extinction-corrected $\mathrm{H} \alpha$ (from the Balmer decrement), and the observed MIR or TIR luminosities. The hybrid tracers combine luminosities measured directly (observed UV or $\mathrm{H} \alpha$ ) with that of the light emitted by dust after being heated by young massive 
stars (see Gordon et al. 2000; Inoue et al. 2001; Hirashita et al. 2003; Iglesias-Páramo et al. 2006; Calzetti et al. 2007; Kennicutt et al. 2007, 2009; Hao et al. 2011; Kennicutt \& Evans 2012; Calzetti 2013; Domínguez Sánchez et al. 2014, for more details).

The most widely used recipes for SFR tracers are included in Calzetti (2013) and are listed here for convenience. These expressions are used to compute the SFR from different data, both for single-band and hybrid recipes, scaled to the same IMF (Kroupa 2001). The mass range varies from 0.1 to $100 M_{\odot}$. The value of the timescale over which the star formation must remain constant depends on each tracer, up to $100 \mathrm{Myr}$ for the UV, MIR or TIR and with a lower value for the $\mathrm{H} \alpha$ tracer, equal, or larger than 6 Myr. The expressions listed below are for global scales, as we are using integrated fluxes for the whole galaxy in each case. Also, recipes for determining the SFR at local scales could be found in the review of Calzetti (2013). For the case of Eqs. (6), (7), and (9), we have rescaled the coefficients that multiply $L(22 \mu \mathrm{m})$ taking into account the $L(25 \mu \mathrm{m}) / L(22 \mu \mathrm{m})$ ratio obtained for our sample and the average ratio between $L(24 \mu \mathrm{m})$ and $L(25 \mu \mathrm{m})$ derived in previous studies (Kennicutt et al. 2009; Calzetti et al. 2010) as explained in Sect. 3.4. The nonlinear behavior for galaxies with $L(22 \mu \mathrm{m})>5 \times 10^{43} \mathrm{erg} \mathrm{s}^{-1}$ present in the original recipe (see Calzetti 2013) is not included here as we only find four galaxies in that range. The total infrared emission in the range $8-1000 \mu \mathrm{m}$ is $L$ (TIR).

First we list those based on single-band, where all the luminosities are in units of $\operatorname{ergs~s}^{-1}$ :

$$
\begin{aligned}
& \operatorname{SFR}\left(M_{\odot} \mathrm{yr}^{-1}\right)=4.6 \times 10^{-44} \times L\left(\mathrm{FUV}_{\text {corr }}\right), \\
& \operatorname{SFR}\left(M_{\odot} \mathrm{yr}^{-1}\right)=5.5 \times 10^{-42} \times L\left(\mathrm{H} \alpha_{\text {corr }}\right), \\
& \operatorname{SFR}\left(M_{\odot} \mathrm{yr}^{-1}\right)=2.8 \times 10^{-44} \times L(\mathrm{TIR}), \\
& \operatorname{SFR}\left(M_{\odot} \mathrm{yr}^{-1}\right)=2.10 \times 10^{-43} \times L(22 \mu \mathrm{m}) .
\end{aligned}
$$

The hybrid tracers are obtained assuming an approximate energy-balance approach. The expressions for the hybrid tracers are shown below where the luminosities are observed and are in units of ergs s${ }^{-1}$. The global coefficients, $4.6 \times 10^{-44}$ and $5.5 \times 10^{-42}\left[M_{\odot} \mathrm{yr}^{-1} / \mathrm{erg} \mathrm{s}^{-1}\right]$, correspond with the calibration of the single-band or monochromatic indicators shown before, UV and $\mathrm{H} \alpha$ respectively. On the other hand, the coefficients that multiply the IR luminosity, either $L(22 \mu \mathrm{m})$ or $L(T I R)$, are dependent on this tracer and on that used for the direct stellar light emission. We empirically calibrate these coefficients in Sect. 4.5.2 to create dust-corrected SFRs, i.e.,

$$
\begin{aligned}
& \operatorname{SFR}\left(M_{\odot} \mathrm{yr}^{-1}\right)=4.6 \times 10^{-44}\left[L\left(\mathrm{FUV}_{\mathrm{obs}}\right)+4.08 \times L(22 \mu \mathrm{m})\right](7) \\
& \operatorname{SFR}\left(M_{\odot} \mathrm{yr}^{-1}\right)=4.6 \times 10^{-44}[L(\mathrm{FUV} \mathrm{obs})+0.46 \times L(\mathrm{TIR})] \\
& \operatorname{SFR}\left(M_{\odot} \mathrm{yr}^{-1}\right)=5.5 \times 10^{-42}\left[L\left(\mathrm{H} \alpha_{\text {obs }}\right)+0.021 \times L(22 \mu \mathrm{m})\right] \\
& \operatorname{SFR}\left(M_{\odot} \mathrm{yr}^{-1}\right)=5.5 \times 10^{-42}\left[L\left(\mathrm{H} \alpha_{\text {obs }}\right)+0.0024 \times L(\mathrm{TIR})\right] .
\end{aligned}
$$

The original recipes for the hybrid tracers make use of the $25 \mu \mathrm{m}$ luminosity, but we are interested in using our $22 \mu \mathrm{m}$ luminosities instead as we have a large number of these measurements. In Sect. 3.4, we justify the use of $L(22)$ instead of $L(25)$ after a $1.05 \pm 0.22$ conversion factor is applied. This factor is computed as the average ratio between $25 \mu \mathrm{m}$ and $22 \mu \mathrm{m}$ luminosities when using high quality $25 \mu \mathrm{m}$ IRAS measurements.

\subsection{SFR surface density}

As mentioned before, the only tracers with enough spatial resolution to compute SFR surface densities across the CALIFA sample are the UV (FWHM $\sim 4.5$ arcsec $)$ and $\mathrm{H} \alpha$

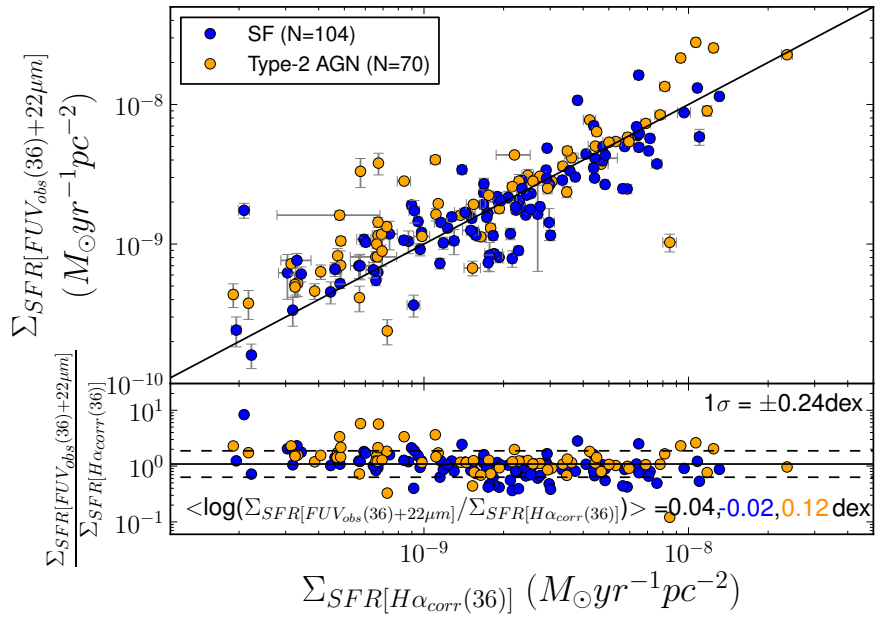

Fig. 6. Star formation rate surface density derived using a combination of observed-FUV and $22 \mu \mathrm{m}$ luminosities compared to Balmer attenuation-corrected $\mathrm{H} \alpha$ star formation rate surface density. The values are obtained within an area of semimajor axis equal to $36 \mathrm{arcsec}$. The solid line corresponds to equal $\Sigma_{\mathrm{SFR}}$ on both axes. The bottom part of this plot represents the residuals as a function of the Balmer-corrected $\mathrm{H} \alpha$ star formation rate surface density. The mean value is shown with the solid line, while dashed lines correspond to the $1 \sigma$ dispersion in dex around the mean value. Black, blue, and orange numbers correspond to the mean values for the whole sample, SF, and type-2 AGN host galaxies, respectively. The tight relation found for these two SFR density tracers shows that there are not systematic differences between them, hence, we can safely use the SFR measurements.

$(F W H M \sim 2.5$ arcsec) measurements. We calculate the SFR surface density in both as the SFR per unit area measured in the largest elliptical apertures (semimajor axis $=36$ arcsec) fitting the PPaK FOV with the ellipticity and PA of the corresponding galaxy. The $\mathrm{H} \alpha$ data are corrected for extinction using the Balmer decrement measured within these apertures. In the case of the UV, we use the hybrid tracer (Eq. (7)). This tracer combines UV-observed luminosities with $22 \mu \mathrm{m}$ luminosities $(F W H M \sim 11$ arcsec). Because of the negative dust extinction gradients found in star-forming galaxies, virtually all the flux at $22 \mu \mathrm{m}$ was found to come from inside these elliptical apertures (see Muñoz-Mateos et al. 2009). Nevertheless, to avoid systematic offsets we decorrected for aperture the total $22 \mu \mathrm{m}$ fluxes using the same aperture correction as described in Sect. 3.1.4. This means that now all the fluxes, $22 \mu \mathrm{m}, \mathrm{H} \alpha$, and FUV, are calculated for the same area.

Thus, the SFR surface density is computed using the following expression:

$$
\Sigma_{\mathrm{SFR}}=\frac{S F R}{\pi a^{2}\left(\frac{d}{206265}\right)^{2}},
$$

where the expressions used for estimating the SFR values are Eqs. (4) and (7); see Sect. 4.1. The parameter $a$ corresponds to the semimajor axis set to 36 arcsec in all cases as described in Sect. 3.1.3 and $d$ is the distance in Mpc to the galaxy calculated from its redshift (listed in Table 1).

Figure 6 compares the hybrid star formation surface density using the observed FUV and $22 \mu \mathrm{m}$ fluxes with their corresponding $\mathrm{H} \alpha$ attenuation-corrected star formation surface density. We have excluded elliptical and lenticular galaxies in this plot where part of the UV emission could come from HB stars responsible for the UV upturn (Brown et al. 1997; Yi et al. 1997). 


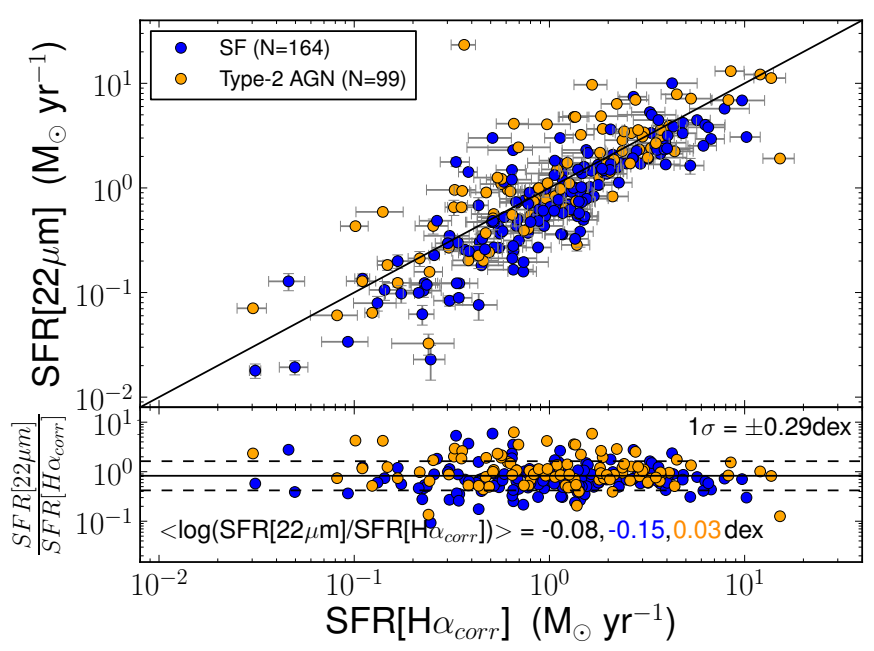

Fig. 7. Comparison between the MIR $(22 \mu \mathrm{m})$ and the Balmer-corrected $\mathrm{H} \alpha$ SFR tracers; recipes from Calzetti (2013); see Eqs. (6) and (4). Solid line corresponds to the 1:1 line. Orange points show type-2 AGN galaxies and blue points represent star-forming galaxies. The legend gives the number of objects available in both cases. Residuals appear in the bottom part of this figure as a function of the Balmer-corrected $\mathrm{H} \alpha$ SFR tracer. Solid line shows the mean value of -0.08 when all the galaxies are included and the dashed lines are referred to the $1 \sigma$ dispersion $( \pm 0.29 \mathrm{dex})$ around it. For SF galaxies, the mean values is -0.15 , while for type-2 AGN host galaxies is 0.03 .

We found a good linear correlation between both measurements in a wide range of values of $\sim 2 \mathrm{dex}$, especially at $\Sigma_{\mathrm{SFR}\left[\mathrm{H} \alpha_{\text {corr }}(36)\right]}>10^{-9} M_{\odot} \mathrm{yr}^{-1} \mathrm{pc}^{-2}$. The mean value of $\left\langle\log \left(\Sigma_{\mathrm{SFR}[\mathrm{FUV}}\right.\right.$ obs $\left.\left.\left.(36)+22 \mu \mathrm{m}\right] / \Sigma_{\mathrm{SFR}\left[\mathrm{H} \alpha_{\text {corr }}(36)\right]}\right)\right\rangle$ is 0.04 and the dispersion is \pm 0.24 dex rms (see Fig. 6 ). There are a number of galaxies at low-surface brightnesses, which correspond to galaxies with null $A(\mathrm{H} \alpha)$ values.

The consistency between the two star formation surface density values and the large range involved shows that there are no systematic differences between the two tracers when SFR surface densities are used or, at least, these are of the order of the object-to-object variation. Thus, from now on we use the SFR instead of SFR density surface, which allows us to use all TIR measurements confidently.

\subsection{Comparison of the different SFR tracers}

As CALIFA provides an excellent $\mathrm{H} \alpha$-integrated luminosity and a precise Balmer decrement we are going to study the SFR tracers found in the literature and provide updated calibrations (Sect. 4.5).

Once we have verified that the extinction-corrected $\mathrm{H} \alpha$ SFR surface density behaves linearly with the hybrid SFR surface density ( $\mathrm{FUV}_{\mathrm{obs}}+22 \mu \mathrm{m}$ ) within the errors (previous section), we can safely assume that any correlation between the integrated SFR is not primarily driven by scaling effects.

Thus, in the rest of Sect. 4 we describe the results from the analysis of the galaxies' total SFR. We first analyze the behavior when using different SFR indicators independently, including the UV and IR-continuum luminosities and, of course, extinction-corrected emission-line $\mathrm{H} \alpha$ luminosity. Then, we compare the results of the different tracers among themselves, assuming that those combining directly observable luminosities (either UV or $\mathrm{H} \alpha$ ) and those associated with dust re-emission (monochromatic or TIR) should be able to recover the entire energy budget from recently-formed massive stars.

\subsubsection{Single-band SFR tracers}

\section{Comparison between Mid-IR and extinction-corrected $\mathrm{H} \alpha$}

In this section, we compare the SFRs using warm-dust sensitive $22 \mu \mathrm{m}$ WISE luminosities with Balmer attenuation-corrected $\mathrm{H} \alpha$ SFRs (Eqs. (6) and (4), respectively) in Fig. 7. We find that at high luminosities $22 \mu \mathrm{m}$ reproduces the SFR measured with extinction-corrected $\mathrm{H} \alpha$. Nevertheless, $L(22 \mu \mathrm{m})$ underestimates the SFR expected at low luminosities, where these galaxies are supposed to have very little dust and consequently weak $L(22 \mu \mathrm{m})$ emission. This could be the reason why the mean value of the residuals, expressed as $\left\langle\log \left(\operatorname{SFR}[22 \mu \mathrm{m}] / S F R\left[\mathrm{H} \alpha_{\text {corr }}\right]\right)\right\rangle$ in the subpanel of Fig. 7, is equal to -0.08 .

We have not included the nonlinear behavior for galaxies with $L(22 \mu \mathrm{m})>5 \times 10^{43} \mathrm{erg} \mathrm{s}^{-1}$ present in the original recipe (see Calzetti 2013), as we only find four galaxies in that range. Three of them have similar values of the $\operatorname{SFR}(22 \mu \mathrm{m}), 11.22$, 12.14 , and 13.07, making this SFR range too small to determine whether a nonlinear fit would be more appropriate in this case.

\section{Comparison between $\beta$-based extinction-corrected UV and extinction-corrected $\mathrm{H} \alpha$}

We analyze the FUV continuum and the $\mathrm{H} \alpha$ emission-line luminosities as tracers of recent star formation (Eqs. (3) and (4)) since both are linked to the presence and amount of massive (i.e., young) stars (see top left panel in Fig. 8). The nonionizing UV emission is mainly photospheric direct emission from O and B stars formed over the past 10-200 Myr and the optical emission lines from ionized gas surrounding massive young stars with lifetimes of $\sim 3-10$ Myr. We apply the attenuation relation given by Eq. (2) mentioned in Sect. 3.2 to correct the FUV luminosity.

We find a rather noisy relation of \pm 0.36 dex around the mean value, $\left\langle\log \left(S F R\left[\mathrm{FUV}_{\text {corr }}\right] / S F R\left[\mathrm{H} \alpha_{\text {corr }}\right]\right)\right\rangle=0.14$. This likely reflects the large uncertainties in the correction for dust attenuation at UV wavelengths using only UV data. They are associated with uncertainties in our knowledge of the slope of the attenuation curve in the UV and with the slope of the underlying stellar continuum. Besides, whether the reddening of the UV continuum can recover all dust-processed SFR is not free for systematics. Figure 8 (top left panel) shows that at high $L(\mathrm{H} \alpha)_{\text {corr }}\left(S F R\left[\mathrm{H} \alpha_{\text {corr }}\right]>5 M_{\odot} \mathrm{yr}^{-1}\right)$ the SFR derived from the UV alone is underestimated. This fact could be explained by taking into account that higher SFRs are associated with higher values of the attenuation (Kennicutt 1998b; Calzetti et al. 2007). It might be that the extinction correction using the FUV-NUV color traces only the most superficial and less extinct part of the SFR. Consequently, the higher SFRs associated with higher values of the extinction are being underestimated.

In order to establish whether other effects could be present, such as an intrinsic discrepancy between the light emitted in the ionizing and nonionizing UV light from galaxy to galaxy, we also compare the SFR $\left[\mathrm{H} \alpha_{\text {obs }}\right]$ and the SFR[FUV $\left.{ }_{\text {obs }}\right]$ in the top right panel of Fig. 8. Although one might think that dust attenuation should erase any linear correlation between these quantities, the fact that one comes from emission from stars and the other from the ionized-gas should partly compensate for the difference in wavelength. In principle, this makes the two quantities not very different for the whole range of SFRs involved with a slope close to unity. We emphasize that this numerical agreement 

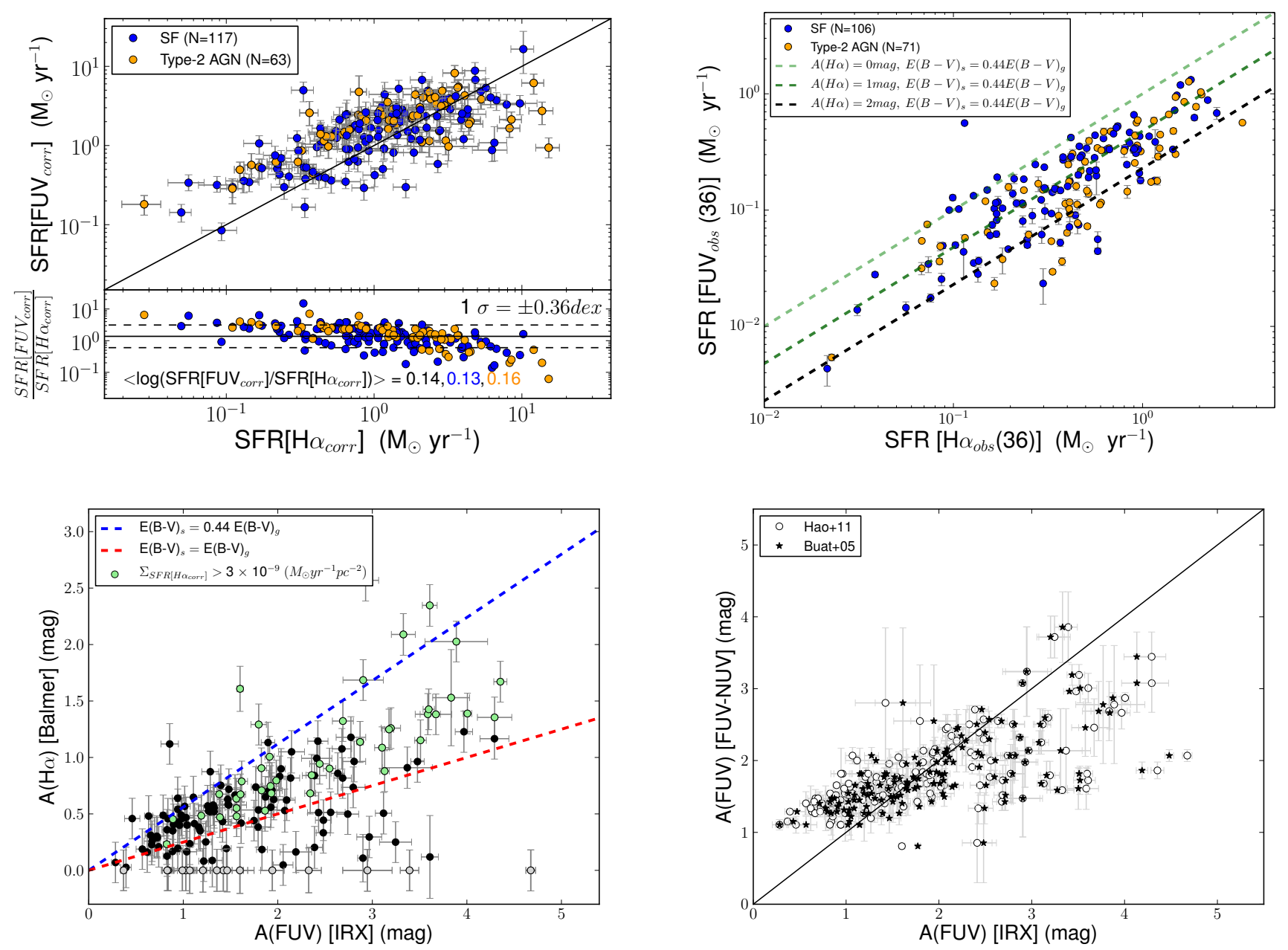

Fig. 8. Top left panel: FUV-corrected SFR as a function of Balmer-corrected H $\alpha$ SFR, using Eqs. (3) and (4), respectively. Color-coding, solid, and dashed lines have the same meaning as in Fig. 7. At high $L(\mathrm{H} \alpha)_{\text {corr }}$ the SFR derived from the UV alone is underestimated. It might be that the extinction correction using the FUV-NUV color traces only the most superficial and less extinct part of the SFR. Consequently, the higher SFRs associated with higher values of the extinction are being underestimated. Top right panel: relation between observed-FUV SFR and observed$\mathrm{H} \alpha$ SFR. Light green, dark green, and black dashed lines correspond to values of $A(\mathrm{H} \alpha)$ equal to 0,1 , and 2 mag, respectively. All cases are based on the assumption that the relation between the color excess of the stars and the gas is $E(B-V)_{\mathrm{s}}=0.44 E(B-V)_{\mathrm{g}}$. Bottom left panel: comparison between $A(\mathrm{H} \alpha)$ from the Balmer decrement (Eq. (1)) and $A(\mathrm{FUV})$ from IR/FUV flux ratio using the expression by Hao et al. (2011). Blue dashed line represents a relation between color excess of $E(B-V)_{\mathrm{s}}=0.44 E(B-V)_{\mathrm{g}}$ while red dashed line assumes that the relation is $E(B-V)_{\mathrm{s}}=E(B-V)_{\mathrm{g}}$. Gray points show cases of $A(\mathrm{H} \alpha)$ equal to zero magnitudes, while light green points show galaxies with the highest values of the SFR surface density. This plot suggest that we might apply a higher value for the relation between the color excess of the gas and the stellar continuum than that found by Calzetti et al. (2000) for our sample, although galaxies with higher values of the SFR surface density are more similar to this previous relation. Bottom right panel: comparison between $A(\mathrm{FUV})$ derived using the FUV-NUV color (Eq. (2)) and $A(\mathrm{FUV})$ from IR/FUV flux ratio. A discrepancy between these two expressions is found for the lowest and highest values of the attenuation. Star-like symbols show the values when the expression used to compute the $A(\mathrm{FUV})$ is that from Buat et al. (2005), while open circles show the values from Hao et al. (2011). The solid line shows the 1:1 line for reference.

does not imply, of course, that there is physical reason for them to be equal in any galaxy.

As these luminosities are observed quantities, we can estimate the expected extinction for these measurements to match. We assume that the color excess of the stellar continuum is related to the color excess of the gas by $E(B-V)_{\mathrm{s}}=0.44 E(B-V)_{\mathrm{g}}$ (Calzetti 1997; Calzetti et al. 2000). For the color excess of the ionized gas we use a standard extinction curve, such as the Galactic extinction curve proposed by Cardelli et al. (1989) and $R_{V}=3.1$. For the case of the color excess of the stellar continuum, we use the attenuation law derived by Calzetti et al. (2000) and $R_{V}=4.05 \pm 0.80$. Finally, we obtain the relation for the stars attenuation in FUV and that of the gas in $\mathrm{H} \alpha$ as $A(\mathrm{FUV})_{\mathrm{s}}=1.79$
$A(\mathrm{H} \alpha)_{\mathrm{g}}$. This value is similar to those obtained by other authors using different samples, such as the case of Hao et al. (2011) that found $A(\mathrm{FUV})_{\mathrm{s}}=1.82 A(\mathrm{H} \alpha)_{\mathrm{g}}$.

If we suppose that the SFR deduced from the FUV continuum and the SFR from $\mathrm{H} \alpha$ emission line (Eqs. (3) and (4) in Sect. 4.1), both corrected by extinction, are equal, then,

$\log \left(\operatorname{SFR}\left[\mathrm{FUV}_{\mathrm{obs}}\right]\right)=\log \left(\operatorname{SFR}\left[\mathrm{H} \alpha_{\mathrm{obs}}\right]\right)+0.4\left[A(\mathrm{H} \alpha)_{\mathrm{g}}-A(\mathrm{FUV})_{\mathrm{s}}\right]$.

Using the previous relation between the corresponding attenuations yields

$\log \left(\operatorname{SFR}\left[\mathrm{FUV}_{\mathrm{obs}}\right]\right)=\log \left(\operatorname{SFR}\left[\mathrm{H} \alpha_{\mathrm{obs}}\right]\right)-0.32 A(\mathrm{H} \alpha)_{\mathrm{g}}$. 
The light green dashed line in Fig. 8 (top right panel) corresponds to values of $A(\mathrm{H} \alpha)$ equal to 0 mag. Nearly every galaxy falls below this line. As expected, having no attenuation correction applied to neither Halpha nor to FUV luminosities implies lower values of the SFR(FUV) as it suffers from higher attenuation. Dark green and black dashed lines in the same figure correspond to values of $\mathrm{H} \alpha$ attenuation of 1 and $2 \mathrm{mag}$, respectively. It seems like values of $A(\mathrm{H} \alpha)$ around $1 \mathrm{mag}$ are in relatively good agreement with our data. However, our $A(\mathrm{H} \alpha)$ values vary from 0 to 2.57 mag (Table 1) with a mean value of 0.49 mag, which does not match the expected value. One possibility for this offset could be that the assumption $E(B-V)_{\mathrm{s}}=0.44 E(B-V)_{\mathrm{g}}$ is not obeyed for our galaxy sample. Alternatively, the corrected SFR could be different when the UV and $\mathrm{H} \alpha$ tracers are used. As we see in Sect. 4.3.2, the latter does not appear to be the cause. In constrast, as pointed out previously, the main aim with this comparison is to show that the real problem when comparing $\mathrm{SFR}\left[\mathrm{H} \alpha_{\text {corr }}\right]$ and SFR[FUV $\left.\mathrm{Forr}\right]$ is the difficult estimation of the $A(\mathrm{FUV})$ and also the importance of the attenuation corrections.

To explore the possibility that our sample might have a different assumption than $E(B-V)_{\mathrm{s}}=0.44 E(B-V)_{\mathrm{g}}$, we compare de $A(\mathrm{H} \alpha)$ from the Balmer decrement and the $A(\mathrm{FUV})[\mathrm{IRX}]$ in the bottom left panel in Fig. 8. The blue dashed line shows the relation between these two quantities when the expression applied for the stellar continuum and the gas color excess is $E(B-V)_{\mathrm{s}}=0.44 E(B-V)_{\mathrm{g}}$. The red dashed line is plotted assuming that the color excess from the stellar continuum and the gas are equal, $E(B-V)_{\mathrm{s}}=E(B-V)_{\mathrm{g}}$. This comparison suggests that we could apply a higher value than that found by Calzetti et al. (2000) for our sample, and that the values of $A(\mathrm{H} \alpha)$ would not be as higher as those expected from the top right panel in Fig. 8. Nevertheless, as we find many points below the blue line that could be due to a deviation from the screen foreground model used to compute the ionized gas extinction, we decided to explore this behavior using another parameter such as the SFR surface density. The light green points show where the galaxies with higher values of the SFR surface density are located in this plot. Clearly, these galaxies are between both lines and they never appear below the red line. This result underscores that galaxies with higher values of SFR surface density (starburst-like) have a relation between the color excess of the stellar continuum and the gas that is more similar to that found by Calzetti et al. (2000) than galaxies with lower values of the SFR surface density.

Finally, we compare $A(\mathrm{FUV})$ derived using the UV-slope (FUV-NUV color) with those obtained using the IR/FUV flux ratio (IRX) in Fig. 8 (bottom right panel). For the IRX case, we use the expression by Buat et al. (2005) (star-like symbols) and that in Hao et al. (2011) (open circles). It is clear from this representation that $A(\mathrm{FUV})[\mathrm{FUV}-\mathrm{NUV}]$ gives higher values than $A$ (FUV)[IRX] for the lowest values of attenuations. On the other hand, $A(\mathrm{FUV})[\mathrm{FUV}-\mathrm{NUV}]$ gives lower values than $A(\mathrm{FUV})[\mathrm{IRX}]$ when the highest values of attenuations are involved. Both expressions, Buat et al. (2005) and Hao et al. (2011), yield similar results. As explained before, this plot suggests that using the FUV-NUV color to recover the dustprocessed SFR is not the best option.

\section{Comparison between TIR and extinction-corrected $\mathrm{H} \alpha$}

The main problem with using the SFR only based on TIR luminosity is that we are assuming that there is a negligible fraction of the light coming directly from the stars without being reprocessed by dust. Besides, even if there are no UV photons escaping directly (without being processed by dust) to the observer,

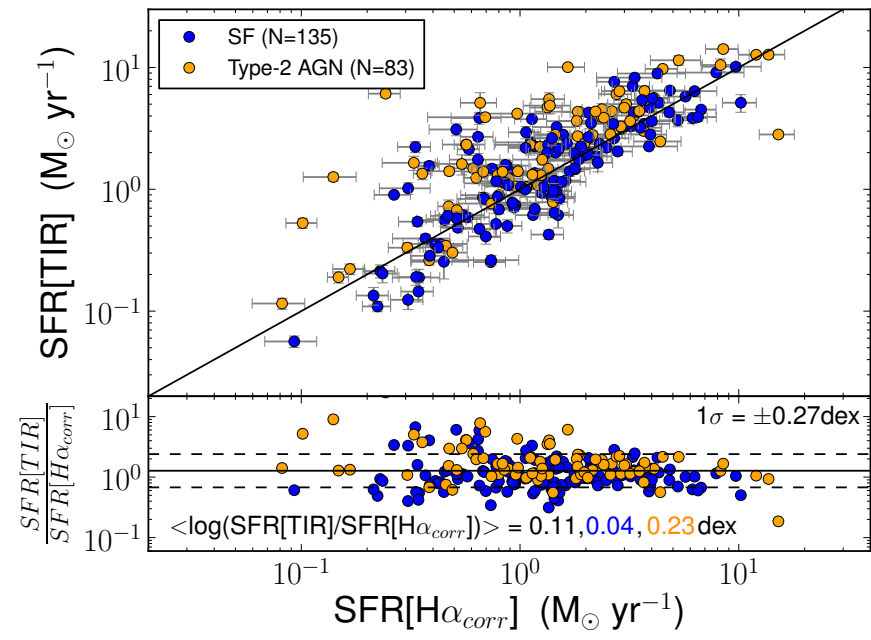

Fig. 9. Comparison between the SFR tracer using the TIR luminosity and the extinction-corrected $\mathrm{H} \alpha$ SFR tracer, after applying Eqs. (5) and (4), respectively. Color-coding, solid, and dashed lines have the same meaning as in Fig. 7.

the calibration of the SFR[TIR] assumes that the light reprocessed by dust comes from young stars, i.e. those linked to the current SF we want to trace. Nevertheless, optical photons from old stars contribute to the heating of the dust (see Johnson et al. 2007) and thus, to the TIR luminosity. Indeed, based on constant star formation (CSF) models, Calzetti (2013) found a reduction in this constant of almost a factor of 2 from models with a CSF lasting for $100 \mathrm{Myr}$ compared to those CSF models lasting for over 10 Gyr. Besides, according to Cortese et al. (2008), for star formation timescales (equivalently the age of the Universe at which the SFR peaks in their "a la Sandage" SFH) larger than $6-7$ Gyr the UV radiation dominates the dust heating with a contribution of $>75 \%$ to the total energy absorbed and then reemitted in the infrared. On the other hand, the same authors derive that if $\tau<5 \mathrm{Gyr}$, the UV light contributes less than $50 \%$ to the TIR emission.

The comparison between the SFR[TIR] and SFR[H $\left.\alpha_{\text {corr }}\right]$ (Fig. 9) shows that at low TIR luminosities the SFR[TIR] are underestimated (in the line of the results of Rieke et al. 2009). We find that for values of the SFR[TIR] below $0.3 M_{\odot} \mathrm{yr}^{-1}$ the average value of $A_{\mathrm{H} \alpha}$ is $0.28 \pm 0.04 \mathrm{mag}$. On the other hand, at high luminosities $\left(S F R\right.$ [TIR] $\left.>1 M_{\odot} \mathrm{yr}^{-1}\right)$ the TIR seems to provide SFRs somewhat higher than those obtained from $\mathrm{H} \alpha$. In fact, a large number of galaxies appear in this regime making the mean value of the ratio between these tracers larger than zero in the residuals, $\left\langle\log \left(S F R[T I R] / S F R\left[\mathrm{H} \alpha_{\text {corr }}\right]\right)\right\rangle=0.11$. This is either because the contribution of heating due to optical photons or nuclear activity becomes relevant at those luminosities and/or because a fraction of the $\mathrm{H} \alpha$ recombination line luminosities are not recovered when correcting for dust attenuation using the Balmer decrement. The analysis of the hybrid calibrations (see section below) favors the former scenario.

\subsubsection{Hybrid SFR tracers}

A powerful way of determining the SFR is to combine a photometric band that is sensitive to the light directly emitted by young stars (i.e., observed UV or $\mathrm{H} \alpha$ fluxes) with that reprocessed by dust, usually in the mid- or far-infrared (or, alternatively, the TIR emission). This is an alternative approach to correct the $\mathrm{UV}$ or $\mathrm{H} \alpha$ fluxes for attenuation. 

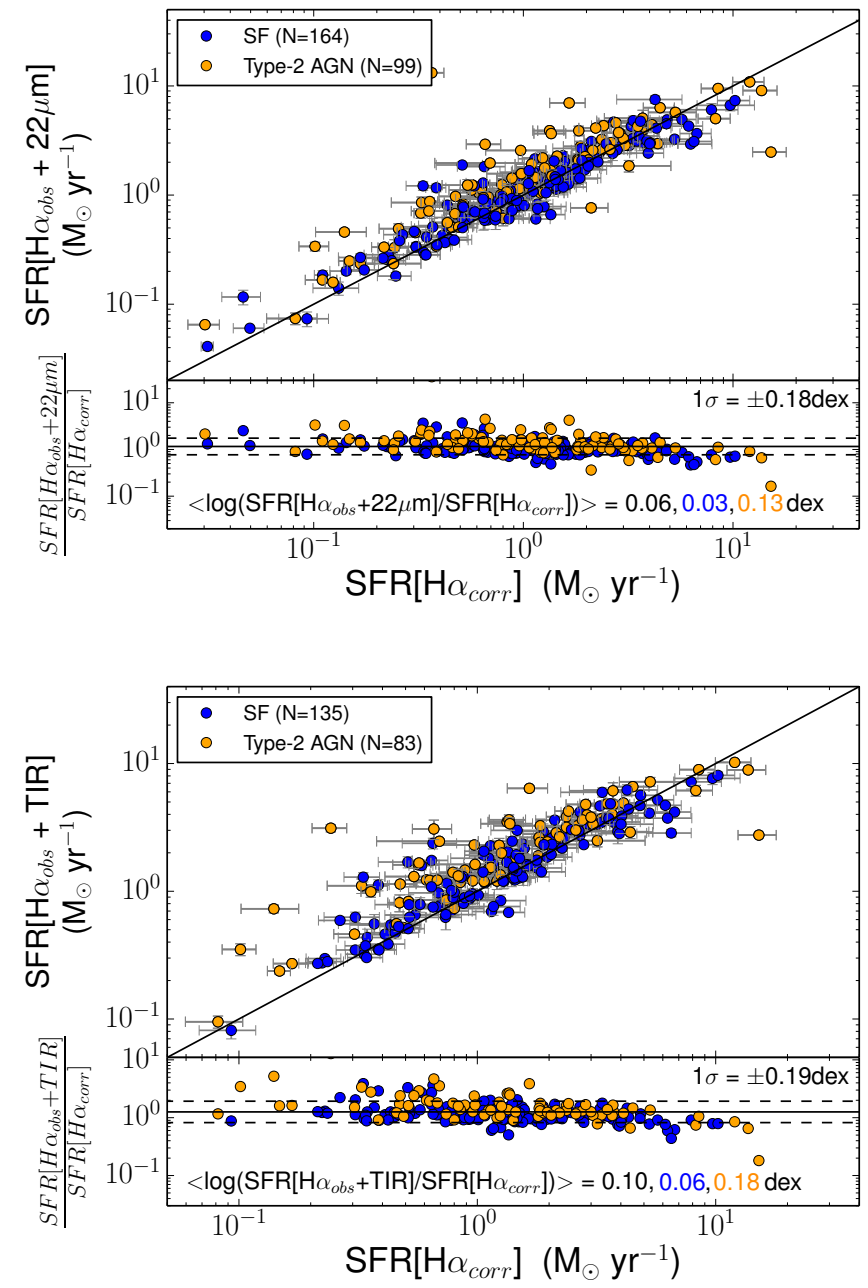

Fig. 10. Top panel: $\mathrm{H} \alpha_{\text {obs }}+22 \mu$ m hybrid tracer as a function of Balmercorrected $\mathrm{H} \alpha$ SFR, using Eqs. (9) and (4), respectively. Color-coding and lines have the same meaning as in Fig. 7. Bottom part shows the residuals as a function of $\mathrm{H} \alpha$-corrected SFR being the mean value 0.06 , 0.03 , and 0.13 for all the galaxies, SF galaxies, and type-2 AGN host galaxies, respectively. Dashed lines represent the $1 \sigma$ dispersion in dex around the mean value. Bottom panel: same as the top panel but showing the $\mathrm{H} \alpha_{\text {obs }}+$ TIR hybrid tracer as a function of Balmer-corrected $\mathrm{H} \alpha$ SFR instead (Eqs. (10) and (4)).

In both cases, the validity of these hybrid tracers is that the observed IR emission comes from light whose optical depth (or attenuation) is of the order of that in the UV or $\mathrm{H} \alpha$, otherwise it would not be possible to write the total SFR as a sum of the two luminosities, observed and dust-processed (see Kennicutt et al. 2009).

This assumption would not be valid if the heating of the dust were dominated by (1) optical photons, which are particularly important at long IR wavelengths where the contribution of lowtemperature dust emission is most relevant; or (2) by UV photons; which are more energetic than those observed directly (e.g., if the bluest observed band is in the NUV; or (3) in the case of a significant AGN contamination, where any of these bands could be actually tracing a UV radiation field that is not merely due to recently-formed massive stars. If there is a significant difference between the $\tau_{\mathrm{FUV}}$ ( or $\tau_{\mathrm{H} \alpha}$ ) and the opacity of the photons that lead to the IR emission used in the corresponding tracer, a linear relation between the SFR and the two (emitted and dustabsorbed) luminosities should not be present. In the particular
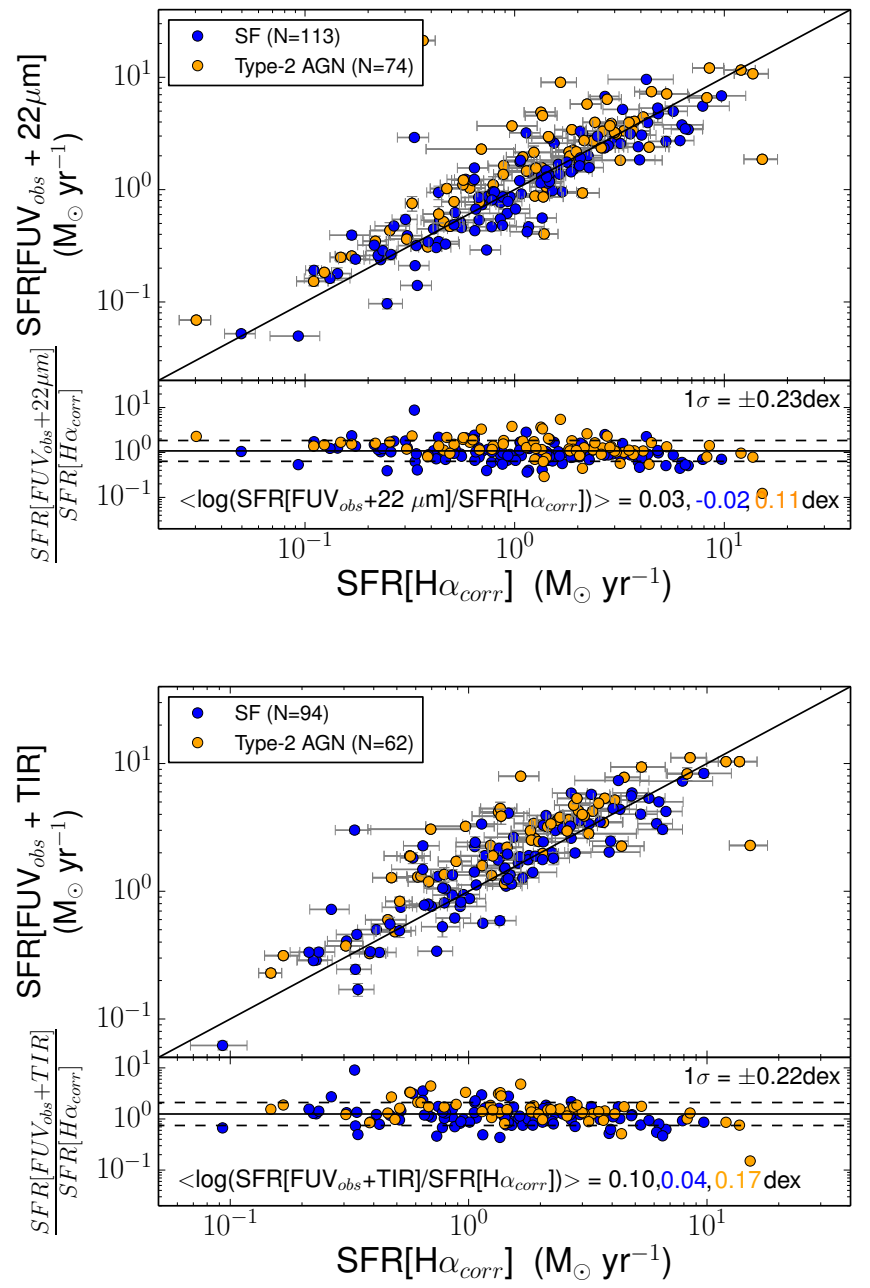

Fig. 11. Top panel: comparison between $\mathrm{FUV}_{\mathrm{obs}}+22 \mu \mathrm{m}$ hybrid tracer and the Balmer-corrected $\mathrm{H} \alpha$ SFR, using Eqs. (7) and (4), respectively. Color-coding, lines, and residuals have the same meaning as in Fig. 10. Bottom panel: same as the top panel, except showing FUV $_{\text {obs }}+$ TIR hybrid tracer as a function of Balmer-corrected H $\alpha$ SFR (Eqs. (8) and (4)). The hybrid tracers shown here and in Fig. 10 reduce the dispersion when compared with single-band tracers.

case of UV and $\mathrm{H} \alpha$, the $\tau$ are similar to the one that comes from the dust component so the approach of using a linear relation between $L\left(\mathrm{FUV}_{\mathrm{obs}}\right)$ or $L\left(\mathrm{H} \alpha_{\mathrm{obs}}\right)$ combined with the $L(\mathrm{IR})$ luminosity can safely be done. That implies that for the IR tracer both bands should suffer the same attenuation (see a detail analysis in Kennicutt et al. 2009; Hao et al. 2011)

Once we have explained the assumptions imposed on the use of the hybrid tracers, we compare them with our $\mathrm{H} \alpha$ extinctioncorrected SFR tracer. In the first place, we examine the behavior using $\mathrm{H} \alpha$ observed luminosity combined with $22 \mu \mathrm{m}$ and TIR luminosity. Figure 10 shows that, applying the method explained in Kennicutt et al. (2009), we now obtain very similar results to theirs but using a larger sample and IFS data for the first time. Secondly, we replace $\mathrm{H} \alpha$ observed luminosity with FUV observed luminosity combined with the IR luminosities (Fig. 11). In both cases, we find a very good correlation across 2.5 dex in SFR, but with an offset in the mean ratio of SFRs of 25 per cent. This offset goes in the sense that SFR derived from the hybrid $\mathrm{H} \alpha+\mathrm{IR}$ and FUV+IR SFR tracers is larger than for the extinction-corrected $\mathrm{H} \alpha$ one. As explained before, one possibility could be the presence of optical photons from old stellar 
populations heating the dust, especially at long IR wavelengths, or the effects of AGN. In particular, if we discriminate between star-forming and type-2 AGN galaxies when computing these ratios, type-2 AGN host galaxies yield larger offsets than those reported for SF galaxies. Section 4.5.2 provides an extensive discussion on this issue.

We conclude that when comparing the hybrid calibrators with Balmer decrement attenuation-corrected $\mathrm{H} \alpha$ SFR tracer, we find tighter correlations than those obtained with singleband tracers (see the $1 \sigma$ dispersions around the mean values in Figs. 7-11).

\subsection{Origin of the discrepancies among SFR tracers}

As we have seen in the previous section, there is generally good agreement between the SFR tracers considered, single-band and especially hybrids, compared to the attenuation-corrected $\mathrm{H} \alpha$ SFR tracer. Nevertheless, we can appreciate some differences if we take a closer look at these relations. In the case of the singleband tracers the main problems appear when using $\mathrm{FUV}_{\text {corr }}$ luminosities as the extinction correction is a big problem to deal with, in particular, for high SFR values. However, we can mitigate this effect using hybrid tracers combining the FUV $\mathrm{obs}_{\text {lumi- }}$ nosity with the IR luminosity, both $22 \mu \mathrm{m}$ and TIR. In the latter case we are assuming that we can recover all the light that it has been re-emitted by the dust. Similar cases appear when using the single-band tracers for $22 \mu \mathrm{m}$ or TIR luminosities, where we apparently lose some SF in galaxies with low values of the SFR. Again, when using hybrid tracers the agreement between calibrators improves.

One of the main reasons behind these discrepancies is the different selection criteria used in the process of determining the SFR calibrators in the literature. Now, we have the opportunity to recalibrate these tracers for a diameter-limited sample of 380 galaxies. Moreover, we are able to use integral field spectroscopy data to assure a proper determination of the attenuation using the Balmer decrement, and thereby, avoiding the problems associated with narrowband imaging. Thus, we are going to provide updated SFR tracers based on our state-of-the-art, attenuation-corrected $\mathrm{H} \alpha$ luminosities.

\subsection{Updated SFR tracers for the diameter-limited CALIFA sample}

We now provide updated calibrations for the global current SFR in external galaxies by means of anchoring the different tracers (single-band and hybrid ones) to the SFR derived from the extinction-corrected $\mathrm{H} \alpha$ luminosity measured in our sample of CALIFA galaxies. Seminal works in this context include Kennicutt (1998a), Kennicutt et al. (2009), and Hao et al. (2011).

As we are interested in calibrating the SFR tracers, we need to exclude galaxies that have type-1 AGN signatures to avoid contamination of sources that are not star-forming (only galaxies UGC 00987 and UGC 03973 are classified as type-1 AGN within our sample). As explained in Sect. 4.3, the information regarding the optical AGN classification can be found in Walcher et al. (2014). We provide separate calibrations for the sample when type-2 AGN galaxies are included and when they are not. The reason for this is that, despite numerous efforts (AlonsoHerrero et al. 2006a; Díaz-Santos et al. 2008, 2010; Castro et al. 2014), the fraction of UV or line emission arising from circumnuclear star formation in type-2 AGN is still highly uncertain. We remind the reader that the nuclear emission in type-2 AGN includes the contribution of both a dusty torus (external radius of a few parsecs, see Ramos Almeida et al. 2009) and a circumnuclear region that could expand up to $1 \mathrm{kpc}$ from the central region. We estimate the level of contamination of the emission from the AGN host galaxies to the total SFR. We find that the contribution of the attenuation-corrected $\mathrm{H} \alpha$ luminosity in the nucleus (measured in a $3^{\prime \prime}$-diameter aperture) over the total for galaxies classified as type-2 AGN is $8.3 \%$, while for the purely SF galaxies this contribution is $5.1 \%$. Galaxies classified as type-2 AGN are shown in our plots as orange points.

We first provide updated calibrations in the case of the single-band tracers. We do not perform this analysis in the case of the SFR[FUV $\left.\mathrm{Forr}_{\text {co }}\right]$ because, as we have explained before, the attenuation correction is highly uncertain and the SFR tracer proposed would not be reliable. On the other hand, we pursue the estimation of the hybrid tracers using $\mathrm{FUV}_{\mathrm{obs}}$ luminosity in Sect. 4.5.2.

\subsubsection{Single-band tracers}

In this section, we provide calibrations for the observed $22 \mu \mathrm{m}$ and TIR luminosities as tracers of the SFR anchoring them to the SFR given by the extinction-corrected $\mathrm{H} \alpha$ luminosity, according to Eq. (4). Figure 12 shows the relation between $L\left(\mathrm{H} \alpha_{\text {corr }}\right)$ and the observed infrared luminosities. We include in these plots both nonlinear ${ }^{4}, \log \left[L\left(\mathrm{H} \alpha_{\text {corr }}\right)\right]=b \times \log \left[\frac{L(\mathrm{IR})}{10^{43}}\right]+\log \left[\frac{a^{\prime}}{5.5 \times 10^{-42}}\right]$ and linear fits, $\log \left[L\left(\mathrm{H} \alpha_{\text {corr }}\right)\right]=\log [L(\mathrm{IR})]+\log \left[\frac{a}{5.5 \times 10^{-42}}\right]$; (see Calzetti 2013). The use of nonlinear calibrations should be restricted to studies using similar (1) selection criteria; (2) apertures; and (3) corrections, or the resulting SFRs could be affected by severe systematics. Linear fits are shown in dashed line, while nonlinear fits are shown in dotted lines. The coefficients for these fits are given in Table 2. In the case of the linear fit, we name the constant $a$, which is expressed in units of $M_{\odot} \mathrm{yr}^{-1} / \mathrm{erg} \mathrm{s}^{-1}$, and for the nonlinear fit we use $a^{\prime}$ because it lacks the physical meaning of $a$. We are going to use $a_{\mathrm{IR}}$ for the hybrid tracers as in this case it is dimensionless and has a different physical meaning than the previous constants (see Sect. 4.5.2 for more details). The values for $a^{\prime}$ and the exponent $b$ are obtained with two different methods, a least-squares linear fit in log scale and a nonlinear least-squares fit using the Python task curve_fit. Both methods yield similar values for these fitting parameters with $22 \mu \mathrm{m}$ and TIR luminosities.

Figure 12 also shows the results of this analysis after including (left panels) or excluding (right panels) type-2 AGN from the sample. In all cases, with and without type-2 AGN and using either the $22 \mu \mathrm{m}$ or TIR luminosity, a nonlinear behavior is clearly present, especially at low luminosities $(\log [L(22 \mu \mathrm{m})]<41.8$ or $\log [L(\mathrm{TIR})]<43.3)$, where most galaxies are located above the best linear fit (see Fig. 12). On the other hand, galaxies with high $22 \mu$ m luminosities $(\log [L(22 \mu \mathrm{m})]>43.4)$ are all found below the linear fit, similar to the behavior observed at $24 \mu \mathrm{m}$ luminosities above $5 \times 10^{43} \mathrm{erg} \mathrm{s}^{-1}$ by Rieke et al. (2009). The bestfitting global slope for our nonlinear SFR calibrations based on $22 \mu \mathrm{m}$ luminosity, $0.733(0.702)$ when type- 2 galaxies are (not) included in the sample, is somewhat smaller (less linear) than the local value ( $500 \mathrm{pc}$ scale) of 0.885 obtained by Calzetti et al. (2007) and than the value of 0.82 given by Cluver et al. (2014) from the analysis of the GAMA survey. Regarding $a^{\prime}$, the values

\footnotetext{
4 We normalize the luminosities to $10^{43} \mathrm{erg} \mathrm{s}^{-1}$ to ensure that the $\mathrm{y}$ intercept for these nonlinear fits is located near the values covered by our sample.
} 

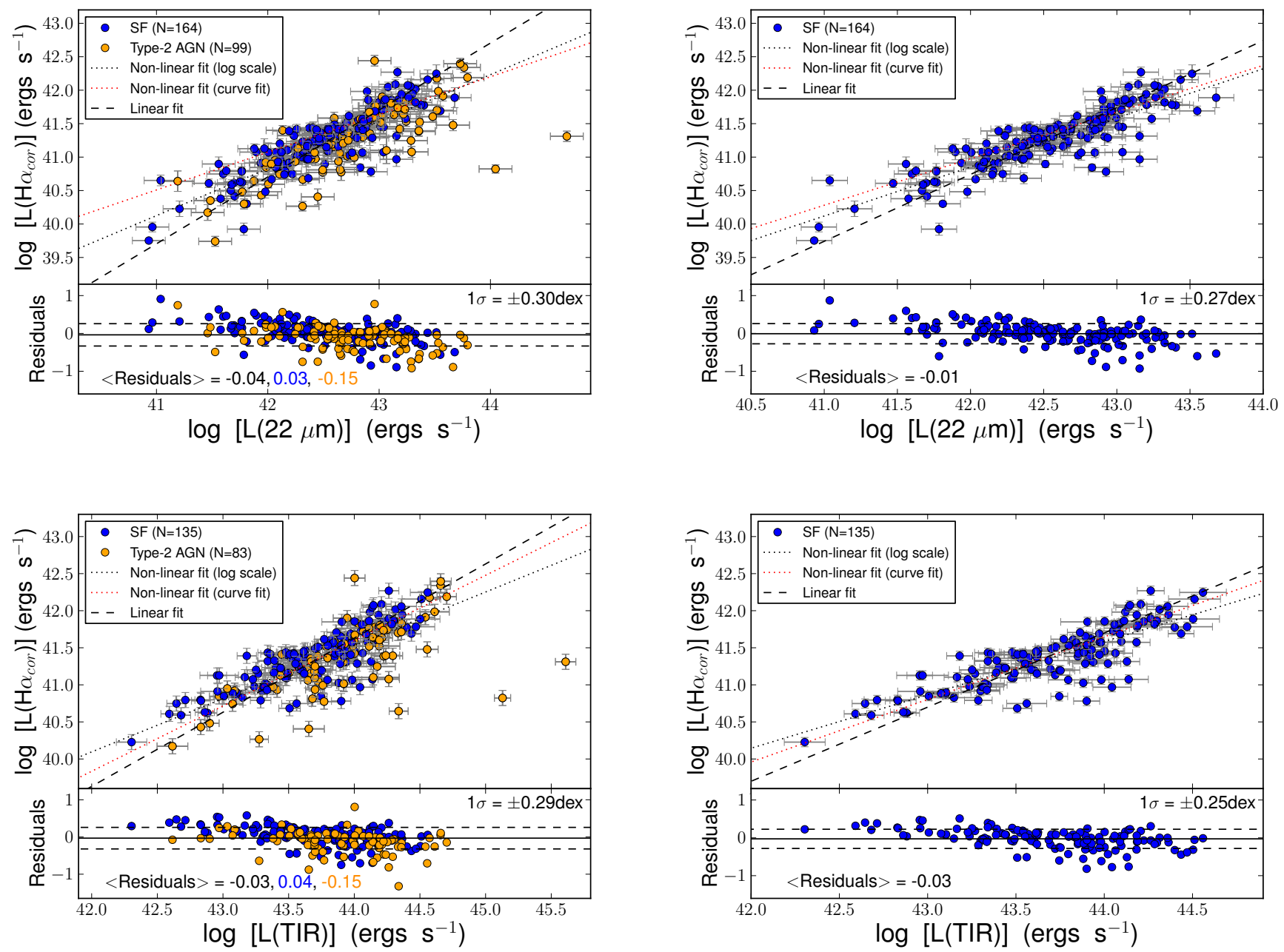

Fig. 12. Updated calibrations for the $22 \mu \mathrm{m}$ and TIR single-band SFR tracers anchoring them to extinction-corrected H $\alpha$ luminosity (Eq. (4)). Orange points in left panels correspond to type-2 AGN, while blue points refer to star-forming galaxies. Linear fits are shown with dashed lines, while nonlinear fits are shown with dotted lines. The results for these calibrations appear in Table 2 . The residuals are computed as the average value of the $\log \left[5.5 \times 10^{-42} \times L\left(\mathrm{H} \alpha_{\text {corr }}\right) / a \times L(\right.$ IR $\left.)\right]$ where $L($ IR) could be $22 \mu \mathrm{m}$ or TIR for the case of the linear fits, after applying a $4 \sigma$ rejection. These values are computed for all galaxies (black), star-forming (blue), and type-2 AGN host galaxies (orange).

Table 2. Values of the $a, a^{\prime}$, and $b$ coefficients for the calibration of single-band tracers: Linear and nonlinear fits, as explained in Sect. 4.5.1.

\begin{tabular}{lcc}
\hline \hline Single-band tracers & Without type-2 AGN & With type-2 AGN \\
\hline SFR $=a \times L(22 \mu \mathrm{m})$ & $a=\left(3.0_{-0.4}^{+2.1}\right) \times 10^{-43}[164]$ & $a=\left(2.8_{-0.6}^{+1.6}\right) \times 10^{-43}[263]$ \\
$S F R=a \times L(\mathrm{TIR})$ & $a=\left(2.8_{-0.5}^{+1.7}\right) \times 10^{-44}[135]$ & $a=\left(2.3_{-0.5}^{+1.6}\right) \times 10^{-44}[218]$ \\
$S F R=a^{\prime} \times\left[L(22 \mu \mathrm{m}) / 10^{43}\right]^{b}(\log$ scale fit) & $a^{\prime}=2.12 \pm 0.18 ; b=0.733 \pm 0.053$ & $a^{\prime}=1.86 \pm 0.11 ; b=0.702 \pm 0.039$ \\
$S F R=a^{\prime} \times\left[L(22 \mu \mathrm{m}) / 10^{43}\right]^{b}($ curve_fit $)$ & $a^{\prime}=2.60 \pm 0.12 ; b=0.697 \pm 0.051$ & $a^{\prime}=2.38 \pm 0.12 ; b=0.564 \pm 0.043$ \\
$S F R=a^{\prime} \times\left[L(\mathrm{TIR}) / 10^{43}\right]^{b}($ log scale fit) & $a^{\prime}=0.403 \pm 0.032 ; b=0.720 \pm 0.042$ & $a^{\prime}=0.359 \pm 0.028 ; b=0.719 \pm 0.038$ \\
$S F R=a^{\prime} \times\left[L(\mathrm{TIR}) / 10^{43}\right]^{b}($ curve_fit $)$ & $a^{\prime}=0.350 \pm 0.063 ; b=0.845 \pm 0.064$ & $a^{\prime}=0.285 \pm 0.054 ; b=0.881 \pm 0.061$ \\
\hline
\end{tabular}

Notes. The numbers in brackets refer to the number of galaxies used in each case (same notation applied in Tables 3-5). The luminosities are expressed in $\mathrm{erg} \mathrm{s}^{-1}$ and the values of the SFR are in $M_{\odot} \mathrm{yr}^{-1}$.

can be very different from those in the literature (even their units are different, obviously) but the uncertainties are of the order of $5-18 \%$, similar to the value quoted by Calzetti (2013), when the log is computed in normalized luminosities. The $22 \mu \mathrm{m}$ or TIR luminosities explored by our sample are significantly lower than those of the sample studied by Rieke et al. (2009), which explains why these authors only needed to make use of a nonlinear fit at their high-luminosity end.
For the case of the linear fit, the difference between the $a$ coefficients with and without type-2 AGN is very small, leading to smaller $a$ coefficient when these objects are included by roughly 7 percent in the case of the $22 \mu \mathrm{m}$ and 18 percent in the TIR. This is likely due to the enhanced contribution of an AGN or, alternatively, obscured circumnuclear star formation to the infrared emission compared to $\mathrm{H} \alpha$. In the former case, the use of this calibration would remove, statistically speaking at least, part of 
the AGN contamination, although some fraction of the H $\alpha$ could still arise from the AGN. Regarding the latter possibility, the use of a calibration anchored to the extinction-corrected $\mathrm{H} \alpha$ luminosity would slightly underestimate the total SFR, as the star formation due to highly-obscured circumnuclear regions in type-2 AGN could be missed. In general, independent of its origin (star formation or not), the total UV light emitted in these regions is hardly recovered using the UV slope or even the Balmer decrement as a measure of its dust attenuation, especially in type- 2 AGN where the BLR is completely hidden from us. The difference here is that if the emission is due to either a BLR or NLR is something that should not be accounted for in terms of the SFR anyway. Finally, we cannot rule out at this stage that, since we are dealing with single-band tracers, this difference arises from a dependence of the attenuation with the level of nuclear activity at a given SFR.

The analysis of the hybrid tracers and the dependence of the $a_{\mathrm{IR}}$ coefficient with the attenuation presented later in this work favors the scenario in which it is the contribution of the AGN itself that leads to these small changes in the single-band SFR calibrations. This will be studied in more detail in a future (spatiallyresolved) analysis. Although, as mentioned before, we will still be unable to disentangle the relative contribution of AGN or circumnuclear star formation to the nuclear emission of type2 AGN host galaxies using these data (see Alonso-Herrero et al. 2006a; Díaz-Santos et al. 2010; Castro et al. 2014, for alternative approaches).

\subsubsection{Hybrid tracers}

In the case of the hybrid indicators we assume a simple energy balance (see Kennicutt et al. 2009, for more details), i.e.,

$$
\begin{aligned}
& \operatorname{SFR}\left(M_{\odot} \mathrm{yr}^{-1}\right)=5.5 \times 10^{-42}\left[L\left(\mathrm{H} \alpha_{\text {obs }}\right)+a_{\mathrm{IR}} \times L(\mathrm{IR})\right] \\
& \operatorname{SFR}\left(M_{\odot} \mathrm{yr}^{-1}\right)=4.6 \times 10^{-44}\left[L(\mathrm{FUV} \text { obs })+a_{\mathrm{IR}} \times L(\mathrm{IR})\right],
\end{aligned}
$$

where $L\left(\mathrm{FUV}_{\mathrm{obs}}\right)$ and $L\left(\mathrm{H} \alpha_{\mathrm{obs}}\right)$ are the observed luminosities in ergs $\mathrm{s}^{-1}$ and $L(\mathrm{IR})$ could be either $L(22 \mu \mathrm{m})$ or $L(\mathrm{TIR})$, also in $\operatorname{ergs~s}^{-1}$.

We calculate the value of the dimensionless $a_{\mathrm{IR}}$ coefficient in the previous hybrid relations as the median of the following ratio for the $L(\mathrm{H} \alpha)$ case (Eqs. (9) and (10)) and for the $L(\mathrm{FUV})$ case (Eqs. (7) and (8)):

$$
\begin{aligned}
a_{\mathrm{IR}} & =\frac{L\left(H \alpha_{\mathrm{corr}}\right)-L\left(\mathrm{H} \alpha_{\mathrm{obs}}\right)}{L(\mathrm{IR})} \\
a_{\mathrm{IR}}= & \frac{\frac{C_{\mathrm{H} \alpha}}{C_{\mathrm{FUV}}} L\left(\mathrm{H} \alpha_{\mathrm{corr}}\right)-L\left(\mathrm{FUV}_{\mathrm{obs}}\right)}{L(I R)},
\end{aligned}
$$

where $C_{\mathrm{H} \alpha}$ and $C_{\mathrm{FUV}}$ are the constants that multiply the $L(\mathrm{H} \alpha)$ and $L\left(\right.$ FUV) in Eqs. (4) and (3) $\left(5.5 \times 10^{-42}\right.$ and $4.6 \times$ $\left.10^{-44}\left[M_{\odot} \mathrm{yr}^{-1} / \mathrm{erg} \mathrm{s}^{-1}\right]\right)$, respectively.

Histograms in Fig. 16 show the distribution of the $a_{\mathrm{IR}}$ coefficient for different hybrid SFR tracers. The end of this section and Sects. 4.5.3 through 4.5.7 give an extensive analysis of the nature of the variation of $a_{\mathrm{IR}}$.

In the case of the combined UV + IR SFR tracers, there are several ways of estimating the calibration. The most common methods are (1) using an energetic balance approach once we have corrected for attenuation in the UV; or (2) anchoring our data to other SFRs measurements.

With regard to (1), we must keep in mind that the estimation of the FUV attenuation is challenging so several methods

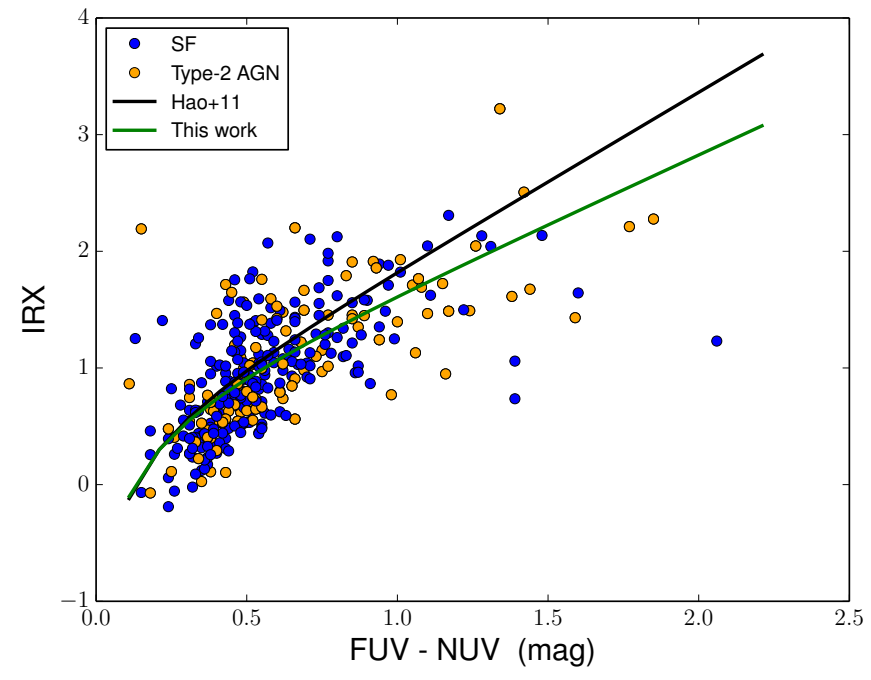

Fig. 13. IRX- $\beta$ relation for the galaxies that have FUV and TIR measurements in the CALIFA mother sample. Blue points represent starforming galaxies while orange points correspond to type-2 AGN host galaxies. Black line shows the fit from Hao et al. (2011), while the green line shows our fit. We obtain a value of $a_{\mathrm{IR}}=0.33 \pm 0.08$ for our fit.

have been put forward for that purpose. The most common approaches are the use of the $\beta$ slope of the UV continuum (similar, but not identical to the FUV-NUV color) or the ratio of IR to UV luminosity. We obtained the FUV-NUV-corrected FUV luminosity (using the $\beta$ slope) in Sect. 4.3.1, but the results show that with this method recovering the SFR is complicated, especially for the highest values of the SFR where the attenuation appears to be underestimated. Other expressions for the FUV attenuation using the FUV-NUV color can be found in the literature (e.g., Kong et al. 2004; Seibert et al. 2005; Salim et al. 2007; Hao et al. 2011). As an example, Hao et al. (2011) use their own expression for the $A(\mathrm{FUV})$ (equation 16 in their paper) to calibrate the TIR + FUV hybrid tracer obtaining that the FUV-NUV-corrected FUV luminosity also underestimates the highest SFRs. As explained before, the other way of deriving the attenuation uses the ratio of IR to UV luminosity. We can use the IRX-corrected FUV luminosity to calibrate the TIR + FUV and the $22 \mu \mathrm{m}+$ FUV hybrid tracers as done in sections 4.1 and 4.3 in Hao et al. (2011). The authors obtain a relation (Eq. (13) in their paper) between the IRX and the FUV-NUV observed color. An important parameter that appears in the former equation is the $a_{\mathrm{IR}}$ value linked directly to the IRX by $A(\mathrm{FUV})=$ $2.5 \log \left(1+a_{\mathrm{IR}} \times 10^{\mathrm{IRX}}\right)$. (See Hao et al. 2011, Eq. (2), where the authors name it $a_{\mathrm{FUV}}$ instead of $a_{\mathrm{IR}}$; we used $a_{\mathrm{IR}}$ for consistency along this work). For the IRX, they use the definition of Meurer et al. (1995): IRX $=\log \left[L(\right.$ TIR $\left.) / L(F U V)_{\text {obs }}\right]$. To see the differences from Hao et al.'s sample and this work, we have derived our own IRX $-\beta$ relation for the galaxies that have FUV and TIR measurements in the CALIFA mother sample, including SF and type-2 AGN host galaxies (see Fig. 13). We use the intrinsic FUV-NUV color obtained by Gil de Paz et al. (2007), $0.025 \pm 0.049 \mathrm{mag}$, which is very similar to that obtained by Hao et al. (2011), $0.022 \pm 0.024$ mag. The black line shows Hao et al.'s fit and the green line is our own fit. This fit gives us a value for the coefficient $a_{\mathrm{IR}}$ of $0.33 \pm 0.08$, in comparison with their value $a_{\mathrm{IR}}=0.46 \pm 0.12$. There is a large dispersion in the previous figure, so even if we account for the fact that our measure of the $a_{\mathrm{IR}}$ coefficient is in good agreement with that found by Hao et al. (2011), we trust method (2) more, and we discuss this next. 
C. Catalán-Torrecilla et al.: Star formation in the local Universe from the CALIFA sample. I.

Table 3. Values of the $a_{\mathrm{IR}}$ coefficients for the calibration of hybrid tracers: global values (see Sect. 4.5.2 for a detailed explanation).

\begin{tabular}{lcc}
\hline \hline Hybrid tracers & Without type-2 AGN & With type-2 AGN \\
\hline$S F R=5.5 \times 10^{-42}\left[L(\mathrm{H} \alpha)_{\mathrm{obs}}+a_{\mathrm{IR}} \times L(22 \mu \mathrm{m})\right]$ & $0.018_{-0.006}^{+0.018}[164]$ & $0.015_{-0.006}^{+0.018}[263]$ \\
$S F R=5.5 \times 10^{-42}\left[L(\mathrm{H} \alpha)_{\mathrm{obs}}+a_{\mathrm{IR}} \times L(\mathrm{TIR})\right]$ & $0.0019_{-0.0005}^{+0.0015}[135]$ & $0.0015_{-0.0006}^{+0.0016}[218]$ \\
$S F R=4.6 \times 10^{-44}\left[L(\mathrm{FUV})_{\mathrm{obs}}+a_{\mathrm{IR}} \times L(22 \mu \mathrm{m})\right]$ & $4.52_{-1.14}^{+3.55}[113]$ & $3.55_{-0.95}^{+3.38}[187]$ \\
$S F R=4.6 \times 10^{-44}\left[L(\mathrm{FUV})_{\mathrm{obs}}+a_{\mathrm{IR}} \times L(\mathrm{TIR})\right]$ & $0.40_{-0.09}^{+0.33}[94]$ & $0.33_{-0.07}^{+0.29}[156]$ \\
\hline
\end{tabular}

Notes. The errors quoted here are the $1 \sigma$ dispersions measured as the interval that includes $68 \%$ of the data points around the median, and correspond with the spread of the histograms in Fig. 16. Note that the standard error of the median, computed from the asymptotic variance formula as $1.253 \times \sigma / \sqrt{N}$, where $\sigma$ is referred to the values listed here and $N$ is the number of galaxies shown in brackets, decreases these errors considerably (black tick marks shown at the top in Fig. 16). The luminosities in these expressions are in erg s${ }^{-1}$ and the values of the SFR are expressed in $M_{\odot} \mathrm{yr}^{-1}$.

Finally, method (2) relies on anchoring the data we want to calibrate to other SFRs measurements, i.e., we establish a reference SFR against which we can compare the hybrid tracers. One possibility would be to use the SFR provided by the extinctioncorrected $\mathrm{Pa} \alpha$ line emission (see Calzetti et al. 2007). This line is only moderately influenced by dust extinction and gives us a good measure of the current SFR. The problems related with this emission line are due to its faintness and to the difficulty of observing a large number of nearby galaxies, as it is only accessible from space.

In our case, we are going to use the extinction-corrected $\mathrm{H} \alpha$ SFR tracer measurements obtained for the first time from IFS data as a reference. These data are required to obtain a proper estimation of the stellar continuum and, therefore, to estimate a reliable measurement of the ionized-gas dust attenuation via the Balmer decrement. Besides, we count on a homogeneous large survey that provides us with good statistics on the properties of nearby star-forming galaxies. For these reasons (and others explained in Sect. 4.4), we consider this tracer as a robust estimator of the SFR. Using this method we obtain the updated calibrations for FUV $+22 \mu \mathrm{m}, \mathrm{FUV}+\mathrm{TIR}, \mathrm{H} \alpha+22 \mu \mathrm{m}$, and $\mathrm{H} \alpha+$ TIR hybrid tracers that appear in Table 3.

The resulting hybrid-tracer calibrations obtained using FUV and $\mathrm{H} \alpha$ as observed luminosities are shown in Figs. 14 and 15. The dispersions found for FUV $+22 \mu \mathrm{m}, \mathrm{FUV}+\mathrm{TIR}, \mathrm{H} \alpha+$ $22 \mu \mathrm{m}$, and $\mathrm{H} \alpha+$ TIR tracers are $0.23(0.19), 0.22$ (0.19), 0.17 (0.14), and 0.17 (0.15) dex when type-2 AGN are (not) included, respectively. The single best-fitting parameter in each of these plots is the median of the distribution of the coefficients that multiply the corresponding infrared luminosity in each galaxy $\left(a_{\mathrm{IR}}\right)$ to match the SFR based on the extinction-corrected $\mathrm{H} \alpha$ luminosity. As explained before, $a_{\mathrm{IR}}$ has been obtained using expressions 16 and 17 and can be found on these plots. The line shown in these figures corresponds to the 1:1 relation in SFR. This line corresponds to the $1: 1$ line also in luminosity in the case of the $\mathrm{H} \alpha+$ IR tracers (Fig. 15) but takes into account the different constant for FUV and $\mathrm{H} \alpha$ given in Eqs. (3) and (4), respectively (Fig. 14). An interesting result found is a nearly constant difference $(\sim 9 \%)$ in the coefficients of the infrared term, $a_{\mathrm{IR}}, 3.55$ (4.52) in the FUV $+22 \mu \mathrm{m}$ and $0.33(0.40)$ in FUV + TIR tracers with (and without) type-2 AGN host galaxies. A $\sim 10 \%$ difference also appears when we compare the $a_{\mathrm{IR}}$ coefficients between the $\mathrm{H} \alpha+22 \mu \mathrm{m}, 0.015$ (0.018) and $\mathrm{H} \alpha+\mathrm{TIR}$, 0.0015 (0.0019), calibrators with (and without) type-2 AGN, respectively. If we compare the ratio between these $a_{\mathrm{IR}}$ coefficients for the combinations of $22 \mu \mathrm{m}$ and TIR data with the luminosity ratio expected for infrared SEDs with different interstellar radiation fields, starlight intensities, dust chemical composition, etc., we can estimate the ratio of the energy absorbed by dust at $\lambda<$ or $>4000$ (see Fig. 2 of Cortese et al. 2008). The most optimal models for carrying out this kind of study, those by Draine \& Li (2007), assume a specific and fixed shape for the interstellar radiation field (the local one) so the effect of optical photons is hidden in the variation of the factor $\gamma$, which parameterize the fraction of dust heated by intense radiation fields. The comparison of the $a_{\mathrm{IR}}$ for $22 \mu \mathrm{m}$ and $a_{\mathrm{IR}}$ for TIR coefficients yields a factor of 0.1 between $L(22)$ and $L(T I R)$, which (according to Fig. 19 of Draine \& Li 2007) corresponds to $\gamma=0.02$, independent of the fractional abundance of PAHs.

In Figs. 14 and 15 we also show the results of this analysis after including (left panels) or excluding (right panels) type-2 AGN from the sample to establish whether the behavior of the hybrid calibrators changes in each case. The four hybrid tracers show the same pattern, the $a_{\mathrm{IR}}$ coefficient decreases when galaxies hosting type-2 AGN are considered. As for the case of the single-band tracers (see Sect. 4.5.1) this decrease in the value of the $a_{\mathrm{IR}}$ coefficient implies that we need to slightly reduce the contribution of the infrared emission in type-2 AGN to match that measured in $\mathrm{H} \alpha$. This implication means that either (1) galaxies hosting type-2 AGN are emitting more light in the infrared, which is not associated with the sites or processes that lead to the $\mathrm{H} \alpha$ emission, both at $22 \mu \mathrm{m}$ and TIR luminosities, as normal star-forming galaxies; or (2) the Balmer-corrected $\mathrm{H} \alpha$ luminosity underestimates the actual SFR in these galaxies.

The distribution of the $a_{\mathrm{IR}}$ coefficient appears in the histograms of Fig. 16 where red dashed lines are referred to its median value. This coefficient has a large dispersion even when only star-forming galaxies are studied. In Table 3 we give the resulting median values of $a_{\mathrm{IR}}$ and the corresponding dispersions (measured as the interval that includes $68 \%$ of the data points around the median). These dispersions appear as red tick marks at the top panels in Fig. 16, while black tick marks indicate the standard error of the median computed from the asymptotic variance formula (which assumes that the underlying distribution is Gaussian) using the previous $1 \sigma$ dispersions. These values are in good agreement with those reported in the literature for integrated measurements of galaxies. Kennicutt et al. (2009) found $0.020 \pm 0.005$ and $0.0024 \pm 0.0006$ for $L(\mathrm{H} \alpha)+a_{\mathrm{IR}} \times L(24 \mu \mathrm{m})$ and $L(\mathrm{H} \alpha)+a_{\mathrm{IR}} \times L(\mathrm{TIR})$, respectively. For the case of the UV luminosity, Hao et al. (2011) found $3.89 \pm 0.15$ and $0.46 \pm 0.12$ for $L(\mathrm{FUV})+a_{\mathrm{IR}} \times L(25 \mu \mathrm{m})$ and $L(\mathrm{FUV})+a_{\mathrm{IR}} \times L(\mathrm{TIR})$, respectively.

In the rest of this section, we study the value of the $a_{\mathrm{IR}}$ coefficient as a function of galaxy properties to obtain insights on the origin of this spread. As we show below, the change in the $a_{\mathrm{IR}}$ coefficient with galaxy properties appears when studying nuclear 

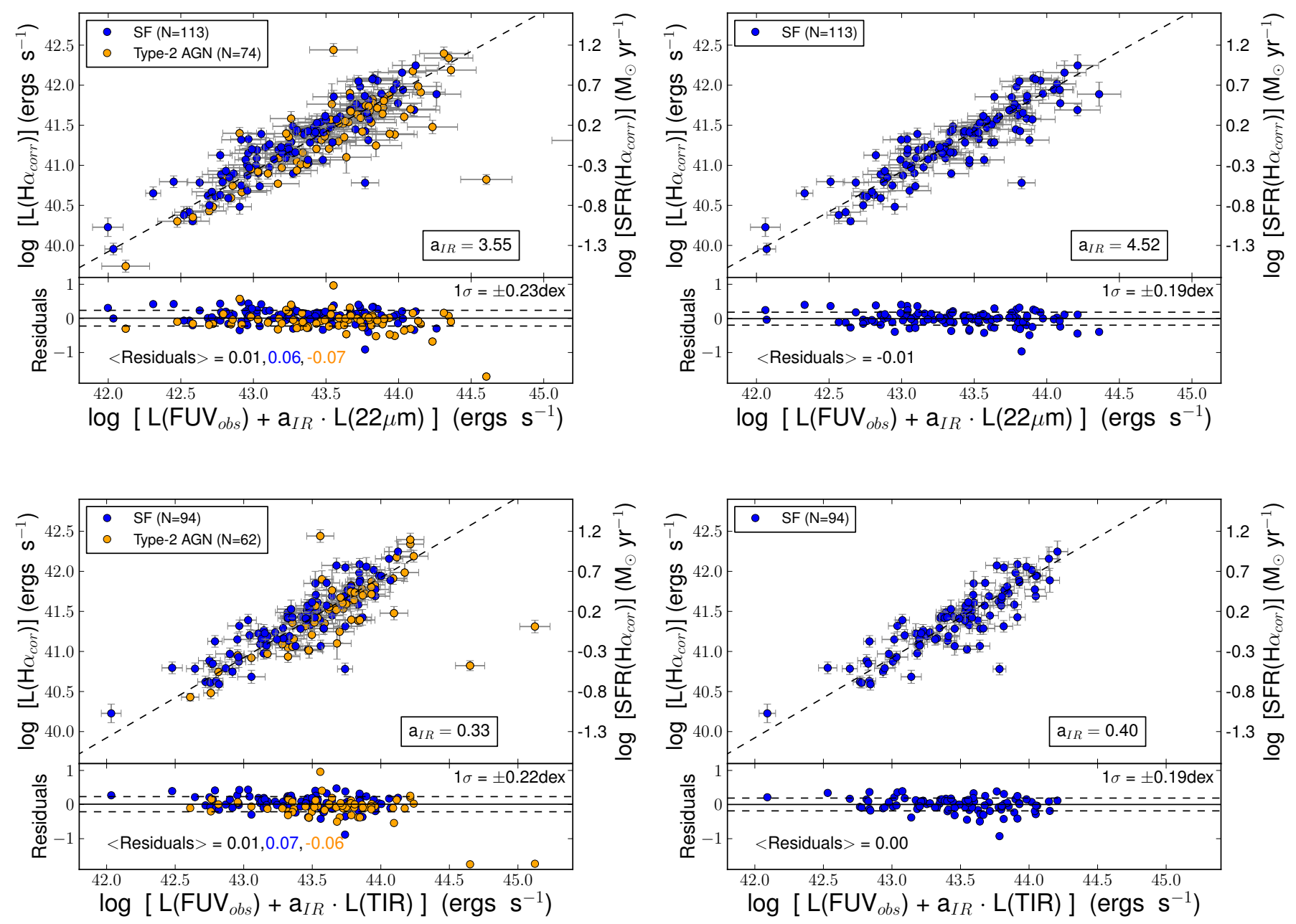

Fig. 14. Updated calibrations for the hybrids tracers that combine FUV observed luminosity and IR luminosity. Top panels show the FUV $\mathrm{obs}+$ $22 \mu \mathrm{m}$ hybrid tracer, while the $\mathrm{FUV}_{\text {obs }}+\mathrm{TIR}$ hybrid tracers appear on the bottom panels. Galaxies hosting type-2 AGN (orange points) are included on the left panels. Blue points refer to star-forming galaxies. Dashed lines correspond to the 1:1 line taking into account the $C_{\mathrm{H} \alpha}$ and $C_{\mathrm{FUV}}$ constants $\left(5.5 \times 10^{-42}\right.$ and $\left.4.6 \times 10^{-44}\right)$ given in Eqs. (3) and (4), respectively. The best-fitting $a_{\mathrm{IR}}$ coefficients calculated as the median value of the expression 17 are shown for clarity. These $a_{\mathrm{IR}}$ values and their corresponding errors appear in Table 3 . The residuals are computed as the average value of the $\log \left[\mathrm{C}_{H \alpha} \times L\left[\mathrm{H} \alpha_{\text {corr }}\right] /\left(C_{\mathrm{FUV}} \times\left(L\left[\mathrm{FUV}_{\mathrm{obs}}\right]+a_{\mathrm{IR}} \times L[\mathrm{IR}]\right)\right)\right]$, where $L(\mathrm{IR})$ could be $22 \mu \mathrm{m}$ or TIR, after applying a $4 \sigma$ rejection. These hybrid tracers show a trend with the $a_{\mathrm{IR}}$ coefficient, so when type-2 AGN host galaxies are included the value of $a_{\mathrm{IR}}$ decreases.

activity as well as galaxy morphology, stellar mass, color, axial ratio, and attenuation.

\subsubsection{Morphological-type dependence of $a_{\mathbb{R}}$ in hybrid tracers}

Given the large number of galaxies in our sample, we can now explore the origin of the differences between the various SFR tracers. In particular, we analyze the origin of the variation of the $a_{\mathrm{IR}}$ with different galaxy properties. Here we focus on the study of its dependence with galaxy morphology (see Walcher et al. 2014). Figure 17 shows the distribution of the $a_{\mathrm{IR}}$ coefficient in bins of morphological type. In the four top plots of this figure, we can see a trend toward the median value of the $a_{\mathrm{IR}}$ coefficient (vertical dashed lines) with the galaxy morphology for $\mathrm{H} \alpha+$ IR tracers. Star-forming galaxies of early type, considered here as S0/a, Sa, and Sab, have lower median values for $a_{\mathrm{IR}}$ (red dashed line) than intermediate-type spirals such as $\mathrm{Sb}$ and $\mathrm{Sbc}$ (gray dashed line). The last group of galaxies, Sc-Sd-SmIrr, shows the largest median value for the $a_{\mathrm{IR}}$ coefficient (blue dashed line). When type-2 AGN galaxies are excluded (right panels in Fig. 17), the trend is less obvious, mainly because of a drastic increase in the median $a_{\mathrm{IR}}$ of early-type spirals.

Regarding the FUV+IR hybrid tracers (four bottom panels in Fig. 17), we find that the median values for $a_{\mathrm{IR}}$ are more similar between S0/a-Sab and Sb-Sbc galaxies. However, the Sc-Sd-SmIrr galaxies still show the highest value for the $a_{\mathrm{IR}}$ coefficient. Table 4 lists the resulting median values and their corresponding errors.

These trends are likely the combination of multiple effects (especially given the large dispersion in the value of $a_{\mathrm{IR}}$ within a given subsample), namely:

(1) The contribution of obscured AGN to the IR luminosity (both at $22 \mu \mathrm{m}$ and in the TIR). This partly explains the fact that the average $a_{\mathrm{IR}}$ decreases when type-2 AGN are included in the sample. The fraction of type-2 AGN is larger within early-type galaxies, so part of the IR luminosity (without an equivalent extinction-corrected $\mathrm{H} \alpha$ luminosity counterpart) is arising from the (obscured) AGN itself. 

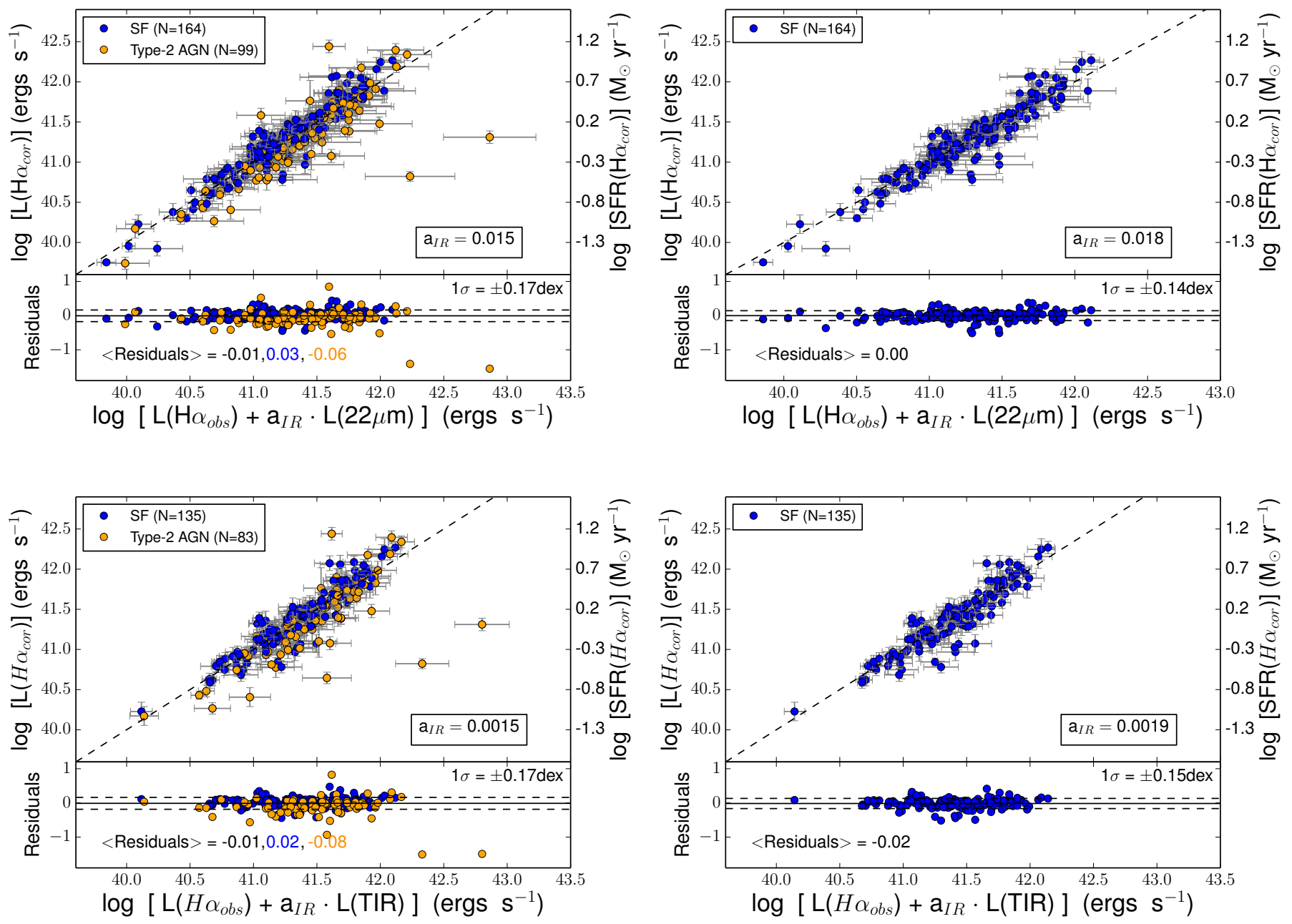

Fig. 15. Updated calibrations for the hybrid tracers, which combine $\mathrm{H} \alpha$ observed luminosity and infrared emission. Those with $22 \mu \mathrm{m}$ luminosity appear at the top, while the tracers that used TIR luminosity are shown at the bottom. Galaxies hosting type-2 AGN (orange points) are included on the left panels. Blue points refer to star-forming galaxies. Dashed lines correspond to the $1: 1$ line. The best-fitting $a_{\mathrm{IR}}$ coefficients calculated as the median value of the expression 16 are shown for clarity. These $a_{\mathrm{IR}}$ values and their corresponding errors appear in Table 3 . The residuals are computed as the average value of the $\log \left[L\left(\mathrm{H} \alpha_{\text {corr }}\right) /\left(L\left(\mathrm{H} \alpha_{\mathrm{obs}}\right)+a_{\mathrm{IR}} \times L(\mathrm{IR})\right)\right]$, where $L(\mathrm{IR})$ could be $22 \mu \mathrm{m}$ or TIR, after applying a $4 \sigma$ rejection. The hybrid tracers show dispersions lower than in the case of the single-band tracers. These hybrid tracers show the same pattern as the ones in Fig. 14, the $a_{\mathrm{IR}}$ coefficient decreases when galaxies hosting type-2 AGN are considered. These calibrations and those in Fig. 14 show that applying an energy balance approximation is a good approach for obtaining reliable SFR tracers for integrated measurements of nearby galaxies. Here, we use IFS data for the first time to achieve this goal.

(2) A fraction of the SFR (that assumed to be in this case accurately measured using a hybrid tracer with a nominal, large, value of the $a_{\mathrm{IR}}$ coefficient) is missed when using the extinction-corrected $\mathrm{H} \alpha$ luminosity. This happens, especially, in early-type spirals so the $a_{\mathrm{IR}}$ coefficient decreases in these objects to compensate for the reduced amount of SFR derived from $\mathrm{H} \alpha$. When the $\mathrm{H} \alpha$ emission missed is exclusively due to an obscured AGN we are in case (1) and $\mathrm{H} \alpha$ would be a fair measure of the SFR.

(3) There is a fraction of the infrared emission that is due to heating by optical photons. One would expect that this effect would be more notorious when the $a_{\mathrm{IR}}$ coefficient refers to the TIR band, as optical photons are expected to heat the dust at low temperatures, where the emission at $22 \mu \mathrm{m}$ is small. As discussed above, the value of $a_{\mathrm{IR}}$ is smaller for S0/a-Sab galaxies, which are galaxies that have older stellar populations and optically bright bulges. Li et al. (2013) also found that the coefficient that multiply the IR luminosity in the $L(\mathrm{H} \alpha)+\mathrm{a} \times L(70 \mu \mathrm{m})$ hybrid tracer is smaller when larger apertures around star-forming regions are used. The authors attribute this effect to the larger associated star formation timescale and the consequent dust heated by old stellar populations.

The fact that by removing type- 2 AGN we reduce but not completely eliminate the morphological-type dependence of $a_{\mathrm{IR}}$ indicates that while (1) appears to have some role, the other possibilities are also at play. Disentangling the contribution of different mechanisms listed above is not easy. In particular, the change observed in $a_{\mathrm{IR}}$ when type- 2 AGN are excluded from the sample could be also because of a decrease in the number of red massive star-forming galaxies in each morphological-type bin. These galaxies are expected to suffer from mechanism (3) as well. The analysis of the variation of $a_{\mathrm{IR}}$ with other properties, mass, color, axial ratio, and ionized-gas attenuation helps us to understand the relative contribution of these mechanisms and, therefore, the specific limitations of the different SFR hybrid tracers. 

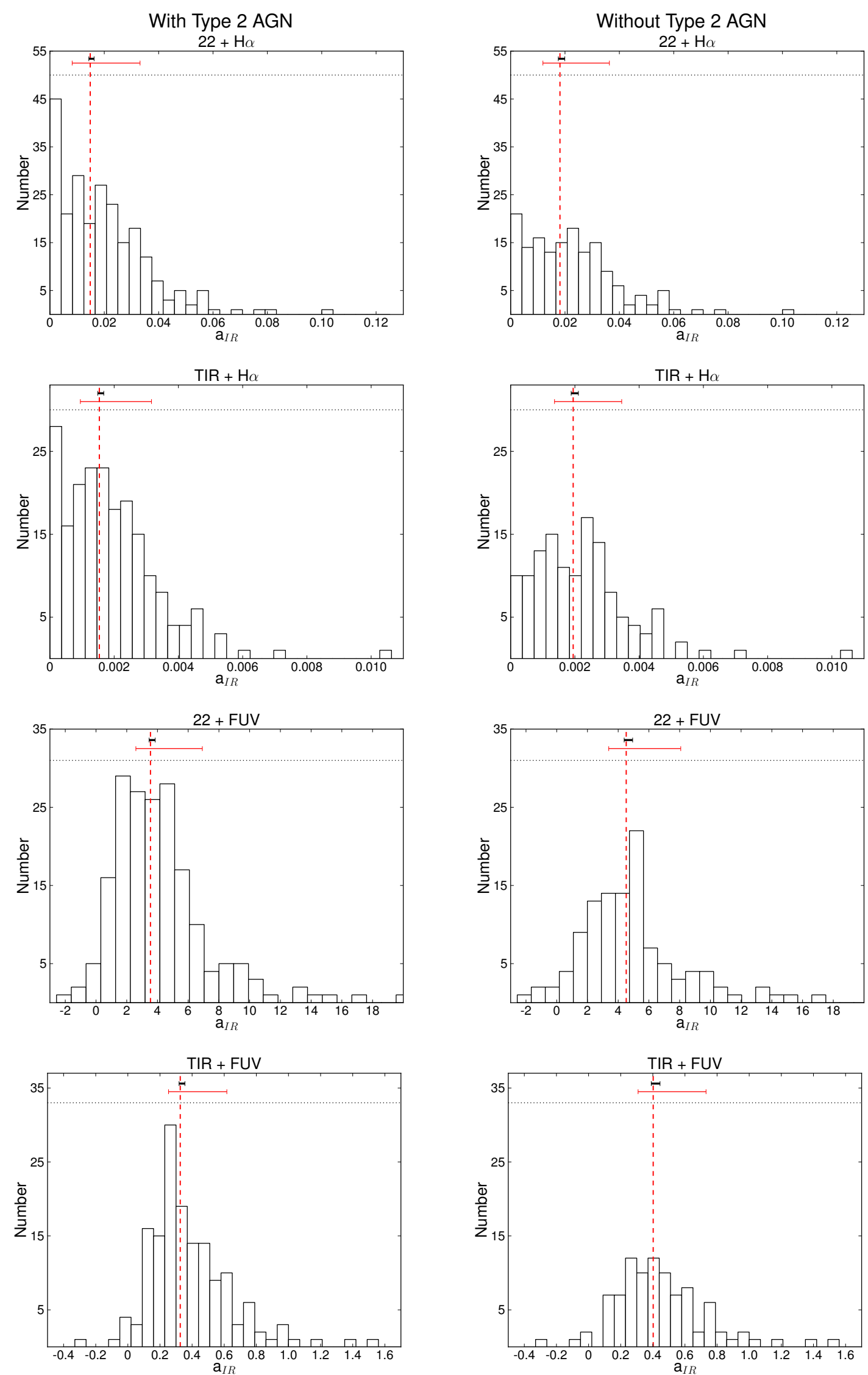

Fig. 16. Histograms showing the distribution of the $a_{\mathrm{IR}}$ coefficient values obtained for the different hybrid tracers. The parameter $a_{\mathrm{IR}}$ is computed using the expressions 16 and 17. The red dashed line corresponds to the median value of this coefficient. The red tick marks shown at the top refers to the $1 \sigma$ dispersions measured as the interval that includes $68 \%$ of the data points around the median quoted in Table 3 , while black tick marks indicate the standard error of the median computed from the asymptotic variance formula using these $1 \sigma$ dispersions. Type- 2 AGN galaxies are excluded from the histograms at right. 
C. Catalán-Torrecilla et al.: Star formation in the local Universe from the CALIFA sample. I.
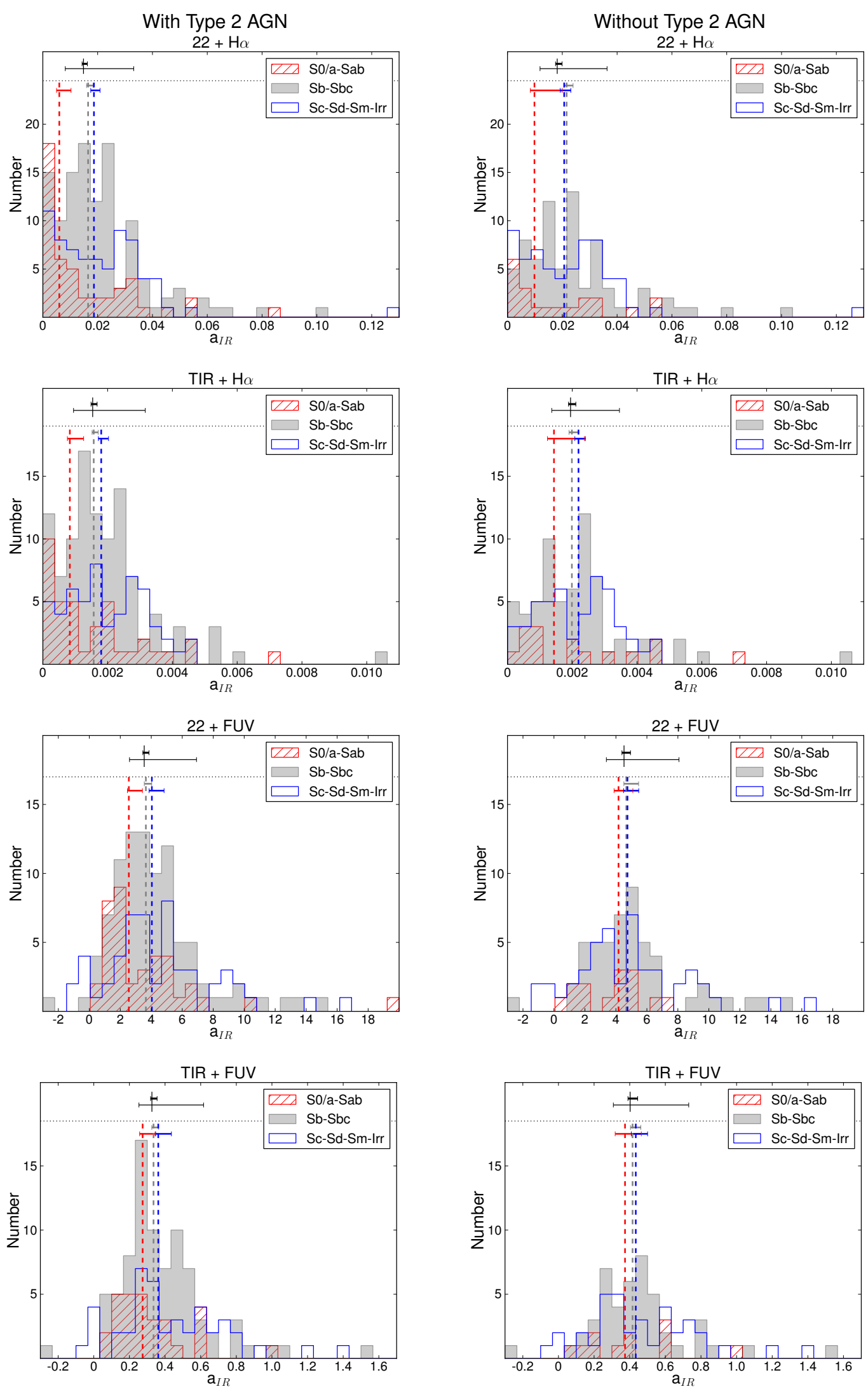

Fig. 17. Four left panels: histograms showing the values of the coefficient that multiply the IR luminosity, $a_{\mathrm{IR}}$, in the hybrid tracers using bins of morphological types. Early-type, star-forming galaxies, considered here as S0/a, Sa, and Sab, are shown in red, intermediate-type spirals such as $\mathrm{Sb}$ and $\mathrm{Sbc}$ appear in gray, and Sc-Sd-Sm-Irr galaxies are represented in blue. Vertical dashed lines correspond to the median value of each galaxy group. Black top marks show the median value for all the galaxies as in Fig. 16. There is a clear trend with the morphological type, late-type galaxies need a higher value of the $a_{\mathrm{IR}}$ coefficient than early-type galaxies. This trend could be explained in terms of the contribution of an obscured AGN, a missing fraction of the $\mathrm{H} \alpha$ extinction-corrected SFR, or heating by optical photons. Four right panels: same histograms as before, except removing the type-2 AGN. There is still a trend with the morphological type, although it is less obvious that in the previous case. 

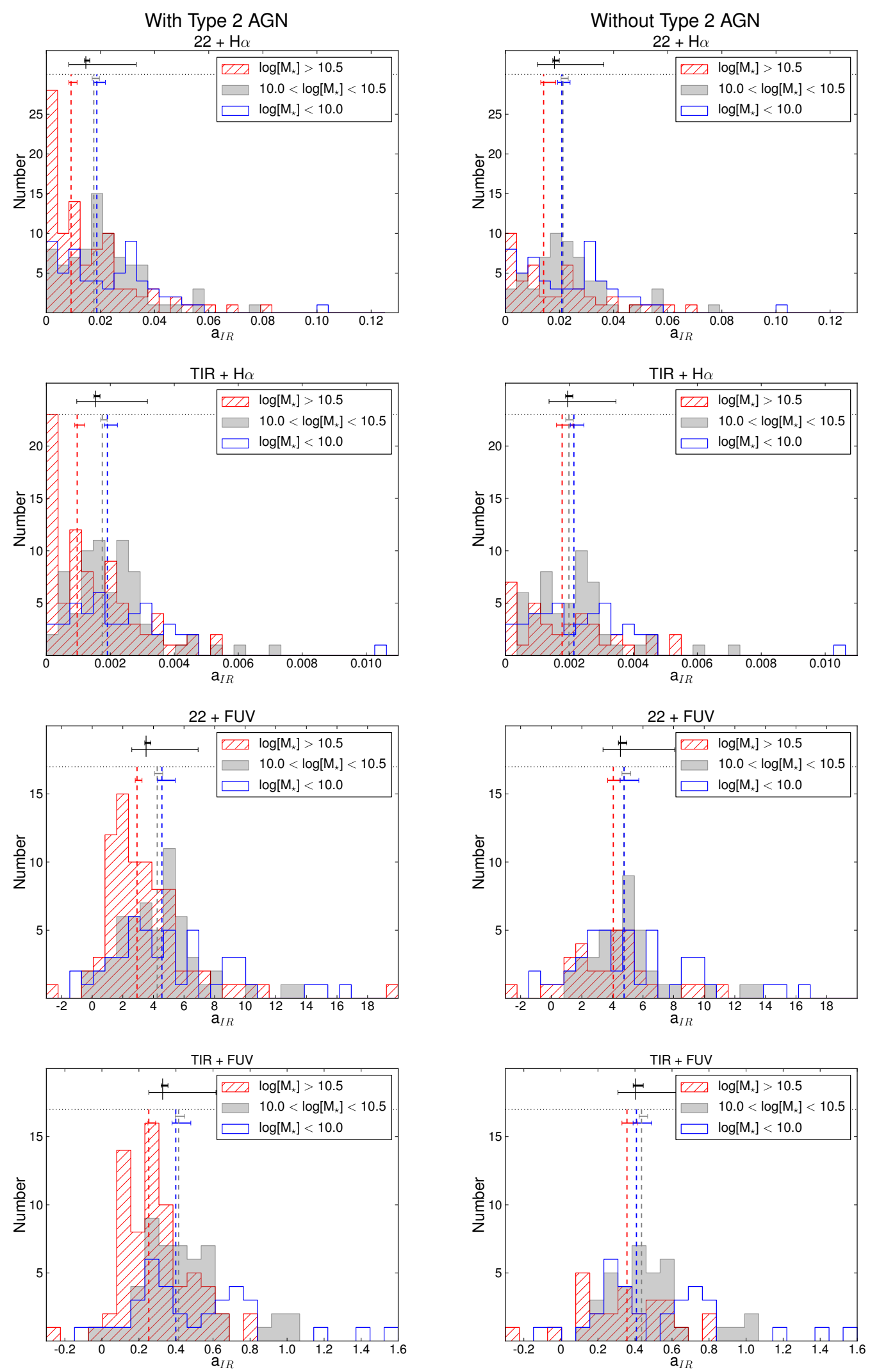

Fig. 18. Left four panels: frequency histograms of $a_{\mathrm{IR}}$ for different hybrid tracers as a function of stellar mass. Massive galaxies $\left(\log \left[M_{*} / M_{\odot}\right]>\right.$ 10.5) appear in red, intermediate-mass galaxies $\left(10<\log \left[M_{*} / M_{\odot}\right]<10.5\right)$ are shown in gray, and low-mass galaxies $\left(\log \left[M_{*} / M_{\odot}\right]<10\right)$ in blue. Dashed vertical lines correspond to the median value of each galaxy group. Black top marks show the median value for all the galaxies as in Fig. 16. There is a clear trend with the stellar mass that less massive galaxies need a higher value of the $a_{\mathrm{IR}}$ coefficient compared with massive galaxies. Right four panels: same as in left panels, except this time type-2 AGNs are excluded for the sample. The distribution of the $a_{\mathrm{IR}}$ coefficient with the stellar mass and morphological type (Fig. 17) allow us to provide, for the first time, a set of hybrid calibrations in terms of these galaxy properties. If the sample to be analyzed is biased toward morphology or, more commonly, luminosity or stellar mass, these tracers would be particularly useful (see Tables 4 and 5). 
C. Catalán-Torrecilla et al.: Star formation in the local Universe from the CALIFA sample. I.

Table 4. Values of the $a_{\mathrm{IR}}$ coefficients for the calibration of hybrid tracers: by morphological type (see Sects. 4.5.2 and 4.5.3 for a detailed explanation).

\begin{tabular}{lcccccc}
\hline \hline \multirow{2}{*}{ Hybrid tracers } & \multicolumn{3}{c}{ Without type-2 AGN } & \multicolumn{3}{c}{ With type-2 AGN } \\
\cline { 2 - 7 } & S0/a-Sab & Sb-Sbc & Sc-Irr & S0/a-Sab & Sb-Sbc & Sc-Irr \\
\hline$L(\mathrm{H} \alpha)_{\text {obs }}+a_{\mathrm{IR}} \times L(22 \mu \mathrm{m})$ & $0.010_{-0.005}^{+0.037}[21]$ & $0.022_{-0.007}^{+0.016}[76]$ & $0.021_{-0.009}^{+0.016}[67]$ & $0.006_{-0.006}^{+0.026}[58]$ & $0.017_{-0.005}^{+0.017}[131]$ & $0.019_{-0.008}^{+0.015}[74]$ \\
$L(\mathrm{H} \alpha)_{\text {obs }}+a_{\mathrm{IR}} \times L(\mathrm{TIR})$ & $0.0014_{-0.0006}^{+0.0029}[16]$ & $0.0020_{-0.0006}^{+0.0013}[68]$ & $0.0022_{-0.0006}^{+0.0012}[51]$ & $0.0008_{-0.0004}^{+0.002}[44]$ & $0.0016_{-0.0004}^{+0.0012}[116]$ & $0.0018_{-0.0005}^{+0.0013}[58]$ \\
$L(\mathrm{FUV})_{\text {obs }}+a_{\mathrm{IR}} \times L(22 \mu \mathrm{m})$ & $4.17_{-0.86}^{+2.76}[14]$ & $4.67_{-0.88}^{+4.52}[51]$ & $4.75_{-1.37}^{+3.97}[48]$ & $2.55_{-0.46}^{+4.37}[39]$ & $3.65_{-0.78}^{+2.90}[93]$ & $4.04_{-1.04}^{+4.61}[55]$ \\
$L(\mathrm{FUV})_{\text {obs }}+a_{\mathrm{IR}} \times L(\mathrm{TIR})$ & $0.37_{-0.15}^{+0.24}[11]$ & $0.42_{-0.06}^{+0.25}[46]$ & $0.43_{-0.12}^{+0.32}[37]$ & $0.27_{-0.07}^{+0.31}[30]$ & $0.34_{-0.07}^{+0.21}[82]$ & $0.36_{-0.08}^{+0.39}[44]$ \\
\hline
\end{tabular}

Notes. The recipes to compute the SFR in $M_{\odot} \mathrm{yr}^{-1}$ shown in the left column are the same as in Table 3 . The luminosities are in units of erg $\mathrm{s}^{-1}$.

Table 5. Values of the $a_{\mathrm{IR}}$ coefficients for the calibration of hybrid tracers: by stellar mass (see Sects. 4.5.2 and 4.5.4 for a detailed explanation).

\begin{tabular}{lcccccc}
\hline \hline \multirow{2}{*}{ Hybrid tracers } & \multicolumn{3}{c}{ Without type-2 AGN } & \multicolumn{3}{c}{ With type-2 AGN } \\
\cline { 2 - 6 } & $\log \left[M_{\star}\right]>10.5$ & $\begin{array}{c}10.0< \\
\log \left[M_{\star}\right]<10.5\end{array}$ & $\log \left[M_{\star}\right]<10.0$ & $\log \left[M_{\star}\right]>10.5$ & $10.0<$ & $\log \left[M_{\star}\right]<10.0$ \\
\hline$L(\mathrm{H} \alpha)_{\text {obs }}+a_{\mathrm{IR}} \times L(22 \mu \mathrm{m})$ & $0.014_{-0.006}^{+0.024}[44]$ & $0.021_{-0.004}^{+0.012}[60]$ & $0.021_{-0.010}^{+0.018}[60]$ & $0.009_{-0.007}^{+0.019}[108]$ & $0.017_{-0.005}^{+0.016}[88]$ & $0.019_{-0.007}^{+0.020}[66]$ \\
$L(\mathrm{H} \alpha)_{\text {obs }}+a_{\mathrm{IR}} \times L(\mathrm{TIR})$ & $0.0018_{-0.0008}^{+0.0017}[37]$ & $0.0020_{-0.0006}^{+0.0008}[54]$ & $0.0021_{-0.0006}^{+0.0017}[44]$ & $0.0010_{-0.0006}^{+0.0018}[92]$ & $0.0018_{-0.0004}^{+0.0011}[77]$ & $0.0019_{-0.0005}^{+0.0018}[48]$ \\
$L(\mathrm{FUV})_{\text {obs }}+a_{\mathrm{IR}} \times L(22 \mu \mathrm{m})$ & $4.06_{-1.61}^{+1.93}[30]$ & $4.75_{-0.63}^{+2.21}[39]$ & $4.77_{-1.39}^{+5.07}[44]$ & $2.93_{-0.84}^{+2.34}[81]$ & $4.25_{-1.09}^{+2.17}[57]$ & $4.55_{-1.56}^{+4.87}[48]$ \\
$L(\mathrm{FUV})_{\mathrm{obs}}+a_{\mathrm{IR}} \times L(\mathrm{TIR})$ & $0.36_{-0.11}^{+0.22}[26]$ & $0.44_{-0.06}^{+0.16}[36]$ & $0.41_{-0.08}^{+0.37}[32]$ & $0.25_{-0.03}^{+0.25}[70]$ & $0.41_{-0.10}^{+0.18}[50]$ & $0.40_{-0.10}^{+0.38}[35]$ \\
\hline
\end{tabular}

Notes. The stellar masses are in units of $M_{\star} / M_{\odot}$. Expressions to compute the SFR $\left(M_{\odot} \mathrm{yr}^{-1}\right)$ appeared in the left column. These recipes are the same as those in Table 3. The luminosities are in units of $\mathrm{erg} \mathrm{s}^{-1}$.

\subsubsection{Stellar mass dependence of $a_{\mathbb{R}}$ in hybrid tracers}

Since morphology alone is not able to establish the origin of the variation of $a_{\mathrm{IR}}$ from galaxy to galaxy and within subsamples, we now explore its dependence with stellar mass. We use the total stellar masses for the CALIFA galaxies from Walcher et al. (2014), Sect. 6.3. (J. Walcher, priv. comm.). The masses are publicly available on the CALIFA DR2 webpage 5 . The procedure for determining them is based on the fitting of UV-opticalNIR SEDs as described in detail in Walcher et al. (2014).

Figure 18 shows the frequency histograms of $a_{\mathrm{IR}}$ for different hybrid tracers (with and without type-2 AGN in the sample) as a function of stellar mass. As for Fig. 17, we find a large dispersion within each mass bin, so clearly mass is not the only driver behind the variation of $a_{\mathrm{IR}}$ from galaxy to galaxy. We find that most massive galaxies $\left(\log \left[M_{*} / M_{\odot}\right]>10.5\right)$ have lower median values of $a_{\mathrm{IR}}$ (red dashed line) than intermediate mass $\left(10<\log \left[M_{*} / M_{\odot}\right]<10.5\right)$ (gray dashed line). In general, lowmass galaxies $\left(\log \left[M_{*} / M_{\odot}\right]<10\right)$ show the largest median value for $a_{\mathrm{IR}}$ (blue dashed line). Table 5 compiles the resulting median values and their corresponding errors.

Besides the relation between mass and color or attenuation, the presence of intense nuclear star formation (such as that found in the classical starburst nuclei, SBN; e.g. Gonzalez-Delgado et al. 1995; Gallego et al. 1996) is also far more common among massive star-forming systems than in low-mass systems (see Pérez-González et al. 2003). It is precisely in these objects where complete obscuration effects in $\mathrm{H} \alpha$ (that would reduce the value $\left.a_{\mathrm{IR}}\right)$ might occur.

Figure 19 compares SFR (derived from the extinctioncorrected $\mathrm{H} \alpha$ luminosity) with the total stellar mass of the galaxies in the sample, the so-called main sequence of galaxies (Brinchmann et al. 2004). This figure shows that type-2 AGN host galaxies (orange dots) dominate the high-mass end

\footnotetext{
5 http://www.caha.es/CALIFA/public_html/?q=content/ califa-2nd-data-release
}

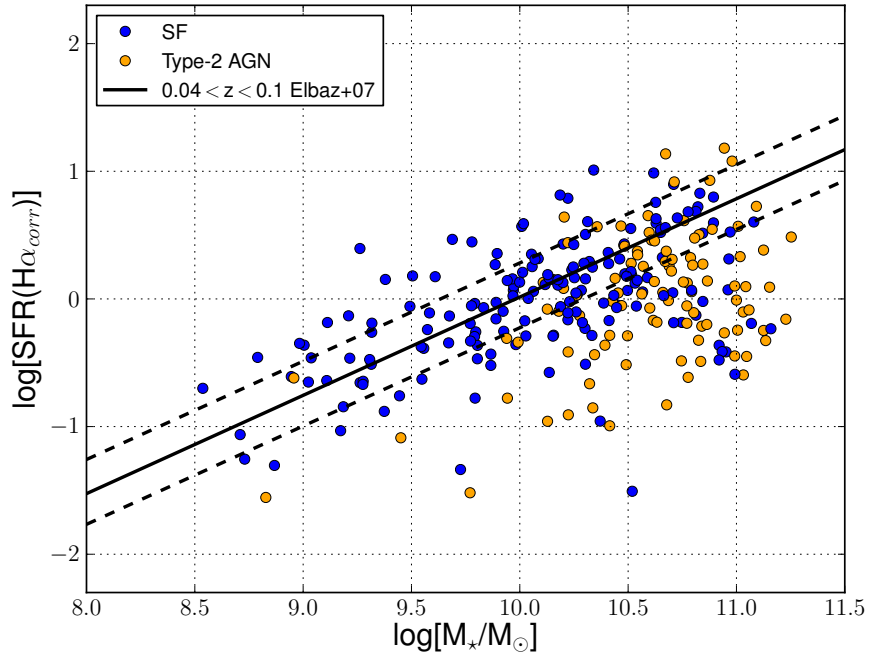

Fig. 19. Correlation between the extinction-corrected $\mathrm{H} \alpha$ SFR and the total stellar mass of the galaxies. Solid line represents the fit of Elbaz et al. (2007) with a slope of 0.77 for galaxies in the $0.04<z<0.1$ range, while dashed lines correspond to the dispersion of this fit. Type2 AGN host galaxies dominate the high-mass end in the main sequence plot for our galaxies. They show somewhat smaller SFR values for the same stellar mass. This fact could be because of a fraction of $\mathrm{H} \alpha$ emission absorbed by the AGN or in the circumnuclear region or, alternatively, the presence of the type-2 AGN might impact the internal evolution of the galaxy quenching the SF.

of those in our diameter-limited sample. Besides, for the same stellar mass, active galaxies show somewhat smaller star formation rates. Some simulations show that when including the AGN feedback, most massive galaxies show a decrease in the specific SFR (Taylor \& Kobayashi 2015). This could be due to a fraction of $\mathrm{H} \alpha$ emission being completely absorbed either at the AGN 

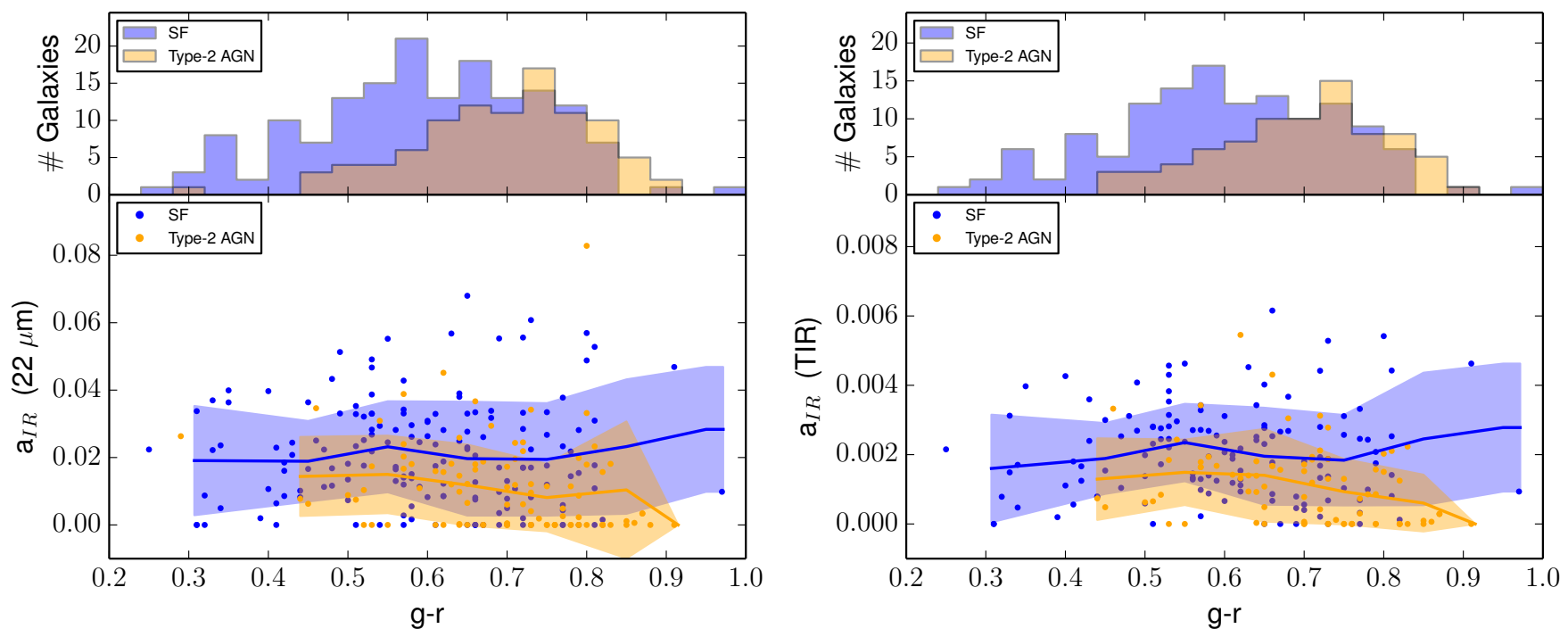

Fig. 20. Left panel: distribution of $a_{\mathrm{IR}}$ coefficients as a function of the galaxies $g-r$ SDSS color for the H $\alpha+22 \mu$ m hybrid tracer. Orange points show type-2 AGN galaxies while blue points represent star-forming galaxies. Filled contours represent the $1 \sigma$ dispersion after applying a $5 \sigma$ rejection around the mean value expressed as a blue (orange) solid line for the SF (type-2 AGN) galaxies. The corresponding histogram with the distribution of the number of galaxies for each $g-r$ SDSS color is plotted on the top for reference applying the same color-coding. Right panel: same as the left panel, except showing the H $\alpha+$ TIR hybrid tracer. There is a clear offset between the star-forming and type-2 AGN host galaxies with the $a_{\mathrm{IR}}$ coefficient at any $g-r$ SDSS color.

or in circumnuclear star formation or to correlations between nuclear activity and other properties, besides mass, such as morphological type or environment. The latter is related with the fact that the presence of an AGN might impact the internal evolution of the galaxy quenching the SF by feedback mechanisms (for a complete review on this topic see Alexander \& Hickox 2012). The analysis of potential effects of the AGN on the current level of star formation at fixed mass (e.g., preference of type-2 AGN for the Green Valley; see Kauffmann et al. 2003; Sánchez et al. 2004) is beyond the scope of this paper.

\subsubsection{Color dependence of $a_{\mathrm{IR}}$ in hybrid tracers}

We address here the dependence of the $a_{\mathrm{IR}}$ coefficient with the color of the integrated stellar population (as traced by the global SDSS $g-r$ color; see Walcher et al. 2014). Figure 20 shows the distribution of $a_{\mathrm{IR}}$ coefficients as a function of the galaxies $g-r$ color in the case of the $\mathrm{H} \alpha+22 \mu \mathrm{m}$ (left panel) and $\mathrm{H} \alpha+\mathrm{TIR}$ (right panel) hybrid tracers. In these plots, type-2 AGN galaxies are shown as orange points and star-forming objects as blue. A clearer picture is obtained when looking separately at star-forming and type-2 AGN galaxies, as traced by the blueand orange-shaded areas in the bottom panels (mean $\pm 1 \sigma$ curves computed after an initial $5 \sigma$ rejection). We see here that most of the decrease in $a_{\mathrm{IR}}$ with color is driven by type-2 AGN host galaxies that appeared to be a little redder than SF galaxies in the top histogram. We find a trend for redder type-2 AGN host galaxies to show a lower value of $a_{\mathrm{IR}}$ especially at colors $g-r>0.6$, although with a large scatter. This trend could be because redder colors are likely related to galaxies with more massive bulges, and these with systems where the IR emission of a (luminous) obscured AGN could effectively dominate over that due to star formation alone. With regard to the pure star-forming galaxies in the sample, we find a relatively flat trend considering the scatter.

\subsubsection{Axial ratio dependence of $a_{\mathrm{IR}}$ in hybrid tracers}

Since highly-inclined systems might be subject to important obscuration effects in the derivation of the SFR, we have explored the dependence between the $a_{\mathrm{IR}}$ coefficient and the axial ratio, as a proxy for the galaxy inclination. Figure 21 shows the histograms of both star-forming and type-2 AGN host galaxies as a function of the axial ratio as given by the RC3 catalog, i.e., measured in the D25 $B$-band isophote. In addition to a clear offset between the two samples at any axial ratio, we find a nearly flat distribution within each sample at axial ratios below $\sim 0.65$. An apparent decrease of $a_{\mathrm{IR}}$ appear for face-on SF systems, although statistics are poor in this case. This is true both for the $\mathrm{H} \alpha+22 \mu \mathrm{m}$ and the $\mathrm{H} \alpha+\mathrm{TIR}$ hybrid tracers (left and right panel in Fig. 21, respectively). We do not find star-forming objects with low axial ratios (where highly-inclined disk galaxies would be located) to show lower $a_{\mathrm{IR}}$. One would expect this if a fraction of the dust-absorbed $\mathrm{H} \alpha$ emission is not recovered by our Balmer decrement based extinction correction (in other words, the $\mathrm{H} \alpha$ emission will be completely obscured.) Therefore, if $\mathrm{H} \alpha$ is missing a fraction of the SFR in some galaxies, these are not necessarily the most inclined systems. Alternatively, missed SFR (if present) could arise from dense nuclear regions, such as (circum)nuclear starbursts.

Prescott et al. (2007) studied the incidence of obscured SF in a large sample of infrared-selected star-forming regions in normal galaxies. They used the $24 \mu \mathrm{m}$ flux as a tracer of the obscured emission due to SF and the uncorrected $\mathrm{H} \alpha$ flux as a tracer of the unobscured portion (the same way we use our $22 \mu \mathrm{m}+\mathrm{H} \alpha$ hybrid tracer, but we compute integrated measurements of galaxies). These authors conclude that the fraction of highly obscured regions in normal, star-forming disk galaxies is small on $500 \mathrm{pc}$ scales. They are more luminous and tend to be closer to the center of the host galaxy. The analysis of obscuration effects is the subject of Sect. 4.5 .7 below which efforts had been made to further explore this issue. 

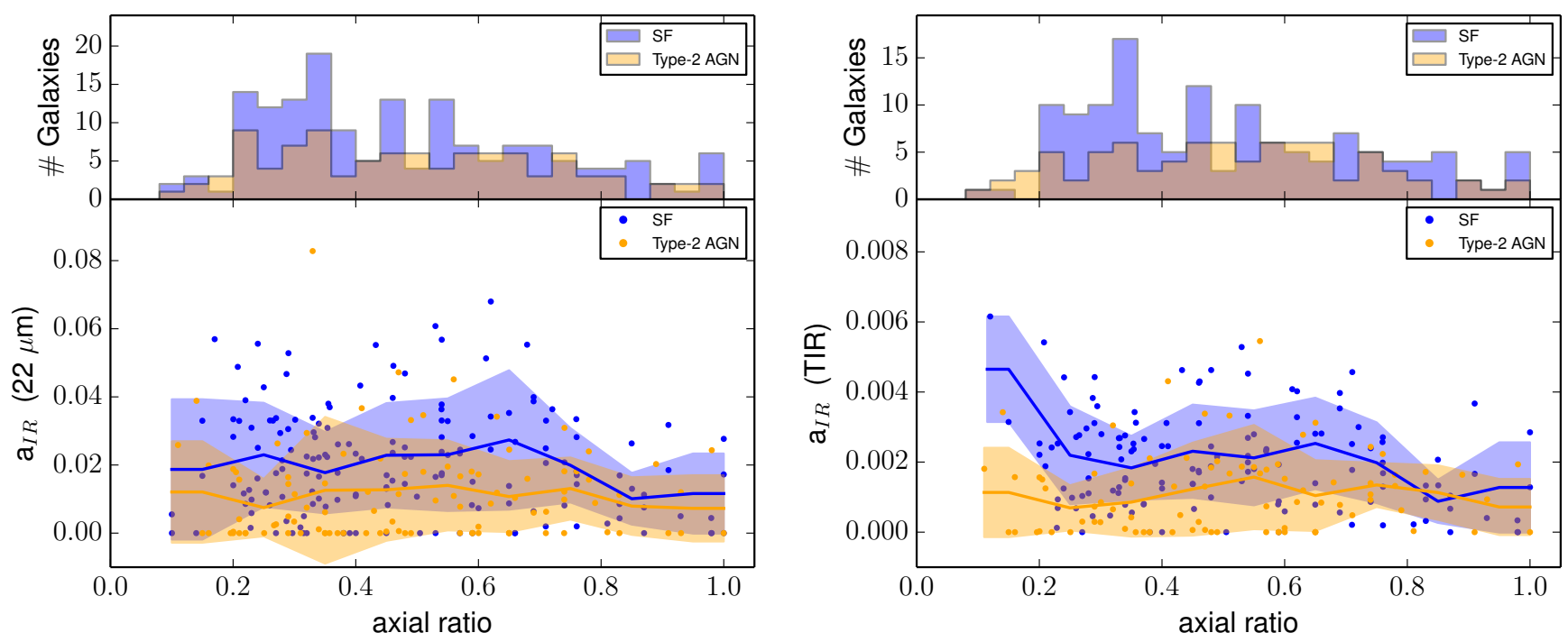

Fig. 21. Left panel: distribution of $a_{\mathrm{IR}}$ coefficients as a function of the galaxies axial ratio for the $\mathrm{H} \alpha+22 \mu \mathrm{m}$ hybrid tracer. Orange points show type-2 AGN galaxies while blue points represent star-forming galaxies. Filled contours represent the $1 \sigma$ dispersion after applying a $5 \sigma$ rejection around the mean value expressed as a blue (orange) solid line for the SF (type-2 AGN) galaxies. The corresponding histogram with the distribution of the number of galaxies for each axial ratio is plotted on the top for reference applying the same color-coding. Right panel: same as the left panel, except showing the H $\alpha+$ TIR hybrid tracer. The SF objects with low axial ratios (where highly-inclined disk galaxies would be located) show similar $a_{\mathrm{IR}}$ values as the rest of the galaxies. Lower $a_{\mathrm{IR}}$ would be expected if a fraction of the $\mathrm{H} \alpha$ emission is completely obscured.

\subsubsection{Attenuation dependence of $a_{\mathbb{R}}$ in hybrid tracers}

To determine whether local obscuration effects in $\mathrm{H} \alpha$ might be behind the decrease of $a_{\mathrm{IR}}$ in galaxies of specific types, masses or colors (as this coefficient should be reduced to compensate by the SFR missed in $\mathrm{H} \alpha$ ), we finally analyze its variation as a function of ionized-gas attenuation. This attenuation is derived from the $\mathrm{H} \alpha / \mathrm{H} \beta$ Balmer decrement as described in Sect. 3.1.4. Figure 22 shows the variation of the $a_{\mathrm{IR}}$ coefficient with the attenuation measured in magnitudes in $\mathrm{H} \alpha$ (the use of log scale in the abscissa is justified by the large concentration of points at low attenuations). The most remarkable feature in this plot is that there is a number of galaxies with low global ionizedgas attenuations that show very small values of $a_{\mathrm{IR}}$. We interpret this as consequence of dust emission that is caused by the heating of photons different from those arising in sites of current star formation. In galaxies where the attenuation derived in $\mathrm{H} \alpha$ is compatible with no attenuation even a small amount of dust emission would lead to a null value for $a_{\mathrm{IR}}$, which results in the number of galaxies with low attenuations and low values of $a_{\mathrm{IR}}$ seen in Fig. 22. These galaxies with very small values of $a_{\mathrm{IR}}$ indicate that, at this level, we are in the limit where $A(\mathrm{H} \alpha)$ can be properly derived, given the low global, ionized-gas attenuations found. The variation of the $a_{\mathrm{IR}}$ coefficient using the FUV attenuation, applying the IRX given by Hao et al. (2011), is shown in Fig. 23 for comparison.

Except for this tail at low attenuation, $A(\mathrm{H} \alpha)<0.2 \mathrm{mag}$, we find no correlation between the two parameters. Should a significant fraction of the SFR being missed when the extinction corrected $\mathrm{H} \alpha$ luminosity is used, one would expect to find a clear decrease in $a_{\mathrm{IR}}$ as the ionized-gas attenuation gets larger. Only when low-mass galaxies are analyzed separately they seem to show a decline in their $a_{\mathrm{IR}}$ values above $A(\mathrm{H} \alpha)=0.4$ mags, although with some discrepant points at $A(\mathrm{H} \alpha)>1$ mag. This decline in $a_{\mathrm{IR}}$ might be due to the fact that in some of these naturally low-metallicity galaxies only, high attenuations are due to the presence of active nuclear star formation events.
Nevertheless, the average and scatter obtained for the $a_{\mathrm{IR}}$ coefficient in these galaxies are not very different from those obtained at higher masses, and do not $a_{\mathrm{IR}}$ reach very low values.

\section{Summary and conclusions}

In this work, we present the analysis of the SFR in a sample of 380 galaxies from the diameter-limited CALIFA survey. A total of 272 galaxies show detected emission in both $\mathrm{H} \beta$ and $\mathrm{H} \alpha$ and are listed in Table 1 for reference. The availability of wide-field IFS for all the galaxies in the sample is a major advantage over other techniques. Using IFS data we can recover the flux in galaxies with low equivalent widths and separate $\mathrm{H} \alpha$ and the [NII] without assuming a [NII]/H $\alpha$ ratio; thus we avoid problems associated with narrowband imaging or long-slit spectroscopy. It also ensures a proper determination of the underlying stellar continuum and, consequently, of the extinction-corrected $\mathrm{H} \alpha$ luminosity.

We combined the aperture-corrected $\mathrm{H} \alpha$ measurements from CALIFA with those measured in other bands, which are also used to estimate the SFR, including luminosity measurements in the UV from GALEX (200 galaxies), $22 \mu \mathrm{m}$ from WISE (265 galaxies), and TIR luminosities from WISE+IRAS+AKARI SED fitting (221 galaxies).

We first compare the extinction-corrected $\mathrm{H} \alpha$ SFR with measurements from single-band (FUV, $22 \mu \mathrm{m}$ and TIR) and hybridtracers $(\mathrm{H} \alpha+22 \mu \mathrm{m}, \mathrm{H} \alpha+\mathrm{TIR}, \mathrm{FUV}+22 \mu \mathrm{m}, \mathrm{FUV}+\mathrm{TIR})$. In this part of the paper, we use recent compilations of SFR recipes by Calzetti (2013). The good correlation between the SFR surface density obtained with extinction-corrected $\mathrm{H} \alpha$ tracer and $\mathrm{FUV}+22 \mu \mathrm{m}$ hybrid tracer guarantees that potential linear correlations between different SFR tracers (some of them not resolved, such as those relying on TIR measurements) are not driven by scaling effects and that global values of the SFR can be used reliably. Our results indicate that, overall, the extinctioncorrected $\mathrm{H} \alpha$ luminosity (once underlying stellar absorption and dust-attenuation effects are properly accounted for) matches the 

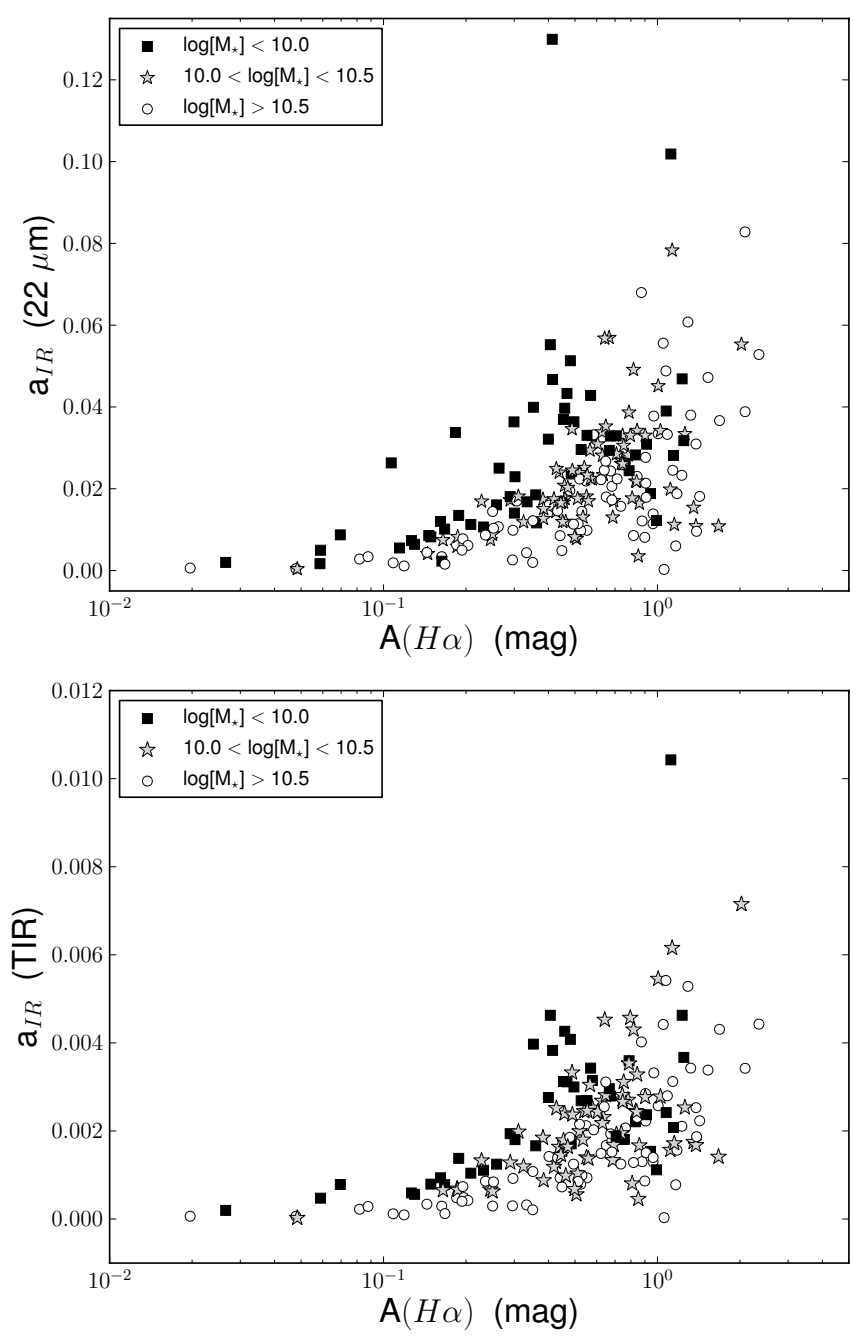

Fig. 22. Top panel: variation of the $a_{\mathrm{IR}}$ coefficient with the $\mathrm{H} \alpha$ attenuation derived using the Balmer decrement for the $\mathrm{H} \alpha+22 \mu \mathrm{m}$ hybrid tracer. Black squares show galaxies with stellar masses lower than $\log \left[M_{\star}\right]<10.0$; gray stars represent galaxies with stellar masses in the range of $10.0<\log \left[M_{\star}\right]<10.5$; and finally, open circles are for the most massive galaxies with $\log \left[M_{\star}\right]>10.5$. Bottom panel: same as previous panel, except this time for the $a_{\mathrm{IR}}$ coefficient, which corresponds to the $\mathrm{H} \alpha+$ TIR hybrid tracer.

SFR obtained from hybrid tracers combining the observed FUV or $\mathrm{H} \alpha$ and the IR ( $22 \mu \mathrm{m}$ and TIR) luminosities with dispersions found around $\sim 0.20 \mathrm{dex}$. In the case of the comparison with single-band tracers, we conclude (1) that the use of IR measurements clearly underestimates the SFR below $\sim 1 M_{\odot} \mathrm{yr}^{-1}$, and (2) the large uncertainty in the correction for attenuation when only FUV-NUV color (similar to the UV slope, $\beta$ ) information is available. This factor introduces a very large scatter, particularly at $S F R>5 M_{\odot} \mathrm{yr}^{-1}$, where the $\beta$-corrected FUV luminosity also tends to underestimate the SFR. This prevents the use of the UV luminosity alone as a SFR tracer.

We also provide a new set of single-band calibrators anchored to the extinction-corrected $\mathrm{H} \alpha$ luminosities. The values for these coefficients appear in Table 2. In the case of the hybrid calibrators, we determine the best (median) fit for the coefficient that weights the amount of IR luminosity reprocessed by dust, $a_{\mathrm{IR}}$. We assume an energetic balance and calculate the $a_{\mathrm{IR}}$ coefficients for different combinations of observed (UV or $\mathrm{H} \alpha$ ) and
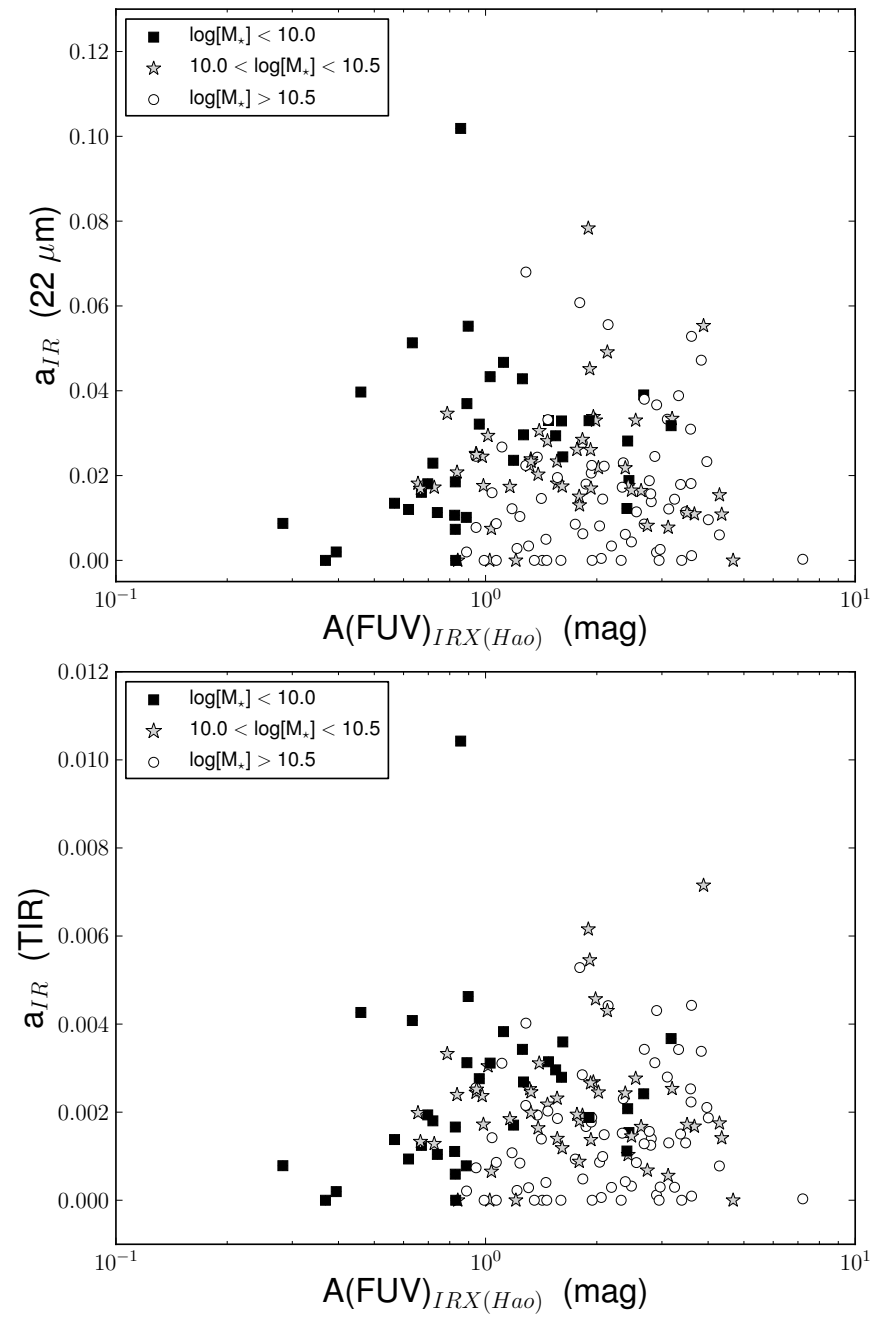

Fig. 23. Top panel: variation of the $a_{\mathrm{IR}}$ coefficient with the FUV attenuation derived using the IRX from Hao et al. (2011) for the $\mathrm{H} \alpha+22 \mu \mathrm{m}$ hybrid tracer. Same symbol-coding as in Fig. 22. Bottom panel: same as previous panel, except this time for the $a_{\mathrm{IR}}$ coefficient that corresponds to the $\mathrm{H} \alpha+\mathrm{TIR}$ hybrid tracer.

dust-reprocessed ( $22 \mu \mathrm{m}$ or TIR) SFR contributions anchored to the extinction-corrected $\mathrm{H} \alpha$ luminosities. These values appear in Table 3 and are calculated with and without galaxies hosting type-2 AGN being considered.

- This analysis allows us to provide, for the first time, a set of hybrid calibrations for different morphological types and masses. These are particularly useful in case that the sample to be analyzed shows a different bias in terms of morphology or, more commonly, luminosity or stellar mass (see Tables 4 and 5).

- We also study the dependence of this coefficient with morphological type and mass and with color (SDSS $g-r$ ), axial ratio, and ionized-gas attenuation.

- The distributions of $a_{\mathrm{IR}}$ values (for each of the hybrid tracers) are quite wide in all cases. While part of the spread can be attributed to changes in morphological type, stellar mass, color, and attenuation among the galaxies in the sample, there is no single physical property that can by itself explain the entire variation in $a_{\mathrm{IR}}$ from galaxy to galaxy.

- The analysis of the dependence of $a_{\mathrm{IR}}$ with galaxy properties indicates that galaxies hosting type-2 AGN tend to reduce the median value of $a_{\mathrm{IR}}$, likely due to the contribution 
of obscured AGN to the infrared emission. The fact that $a_{\mathrm{IR}}$ does not show a particularly low value at high ionized-gas attenuations nor low axial ratios, suggests that obscured star formation is, comparatively, playing a minor role. Part of the dependence of the median value of $a_{\mathrm{IR}}$ with the morphological type disappears once the AGN contribution is removed, although early spirals still show a somewhat lower $a_{\mathrm{IR}}$ than intermediate- and late-type spirals. This behavior, also present when comparing massive with less massive systems, can be explained in part as due to the enhanced contribution of optical photons to the heating of the dust in both early-type spirals and massive systems.

These conclusions will allow us to make use of the CALIFA IFS data to explore the distribution of the SFR with spatial resolution in a future work (see Catalán-Torrecilla et al. 2014). We emphasize that the impact of potential differences in the selection criteria should be addressed carefully when extrapolating these results to other samples of galaxies and, particularly, to other redshifts.

Acknowledgements. This study makes uses of the data provided by the Calar Alto Legacy Integral Field Area (CALIFA) survey (http://califa.caha. es). CALIFA is the first legacy survey being performed at Calar Alto. The CALIFA collaboration would like to thank the IAA-CSIC and MPIA-MPG as major partners of the observatory, and CAHA itself, for the unique access to telescope time and support in manpower and infrastructures. The CALIFA collaboration also thanks the CAHA staff for the dedication to this project. C.C.-T. thanks the support of the Spanish Ministerio de Educación, Cultura y Deporte by means of the FPU fellowship program. The authors also thank the support from the Plan Nacional de Investigación y Desarrollo funding programs, AYA2012-30717 and AyA2013-46724P, of Spanish Ministerio de Economía y Competitividad (MINECO). P.G.P.-G. acknowledges support from the AYA2012-30717 and AYA2012-31277. J.I.P. acknowledges financial support from the Spanish MINECO under grant AYA2010-21887-C04-01 and from Junta de Andalucía Excellence Project PEX2011-FQM7058. R.A.M. is funded by the Spanish program of International Campus of Excellence Moncloa (CEI). M.A.P.T. acknowledges support from the Spanish MINECO through grant AYA2012-38491-C02-02. A.d.O. acknowledge financial support from the Spanish grant AYA2013-42227-P. Support for L.G. is provided by the Ministry of Economy, Development, and Tourism's Millennium Science Initiative through grant IC120009, awarded to The Millennium Institute of Astrophysics, MAS. L.G. acknowledges support by CONICYT through FONDECYT grant 3140566. J.M.G. acknowledges support from the Fundação para a Ciência e a Tecnologia (FCT) through the Fellowship SFRH/BPD/66958/2009 from FCT (Portugal) and POPH/FSE (EC) by FEDER funding through the program Programa Operacional de Factores de Competitividade (COMPETE). J.M.G. also acknowledges support by FCT under project FCOMP-01-0124FEDER-029170 (Reference FCT PTDC/FIS-AST/3214/2012), funded by FCTMEC (PIDDAC) and FEDER (COMPETE). This research has made use of the NASA/IPAC Extragalactic Database (NED) which is operated by the Jet Propulsion Laboratory, California Institute of Technology, under contract with the National Aeronautics and Space Administration. This publication makes use of data products from the Wide-field Infrared Survey Explorer, which is a joint project of the University of California, Los Angeles, and the Jet Propulsion Laboratory/California Institute of Technology, funded by the National Aeronautics and Space Administration. This research has made use of the NASA/ IPAC Infrared Science Archive, which is operated by the Jet Propulsion Laboratory, California Institute of Technology, under contract with the National Aeronautics and Space Administration. Funding for the SDSS, and SDSS-II has been provided by the Alfred P. Sloan Foundation, the Participating Institutions, the National Science Foundation, the US Department of Energy, the National Aeronautics and Space Administration, the Japanese Monbukagakusho, the Max Planck Society, and the Higher Education Funding Council for England. The SDSS Web Site is http://www.sdss.org/. The SDSS is managed by the Astrophysical Research Consortium for the Participating Institutions. The Participating Institutions are the American Museum of Natural History, Astrophysical Institute Potsdam, University of Basel, University of Cambridge, Case Western Reserve University, University of Chicago, Drexel University, Fermilab, the Institute for Advanced Study, the Japan Participation Group, Johns Hopkins University, the Joint Institute for Nuclear Astrophysics, the Kavli Institute for Particle Astrophysics and Cosmology, the Korean Scientist Group, the Chinese Academy of Sciences (LAMOST), Los Alamos National
Laboratory, the Max-Planck-Institute for Astronomy (MPIA), the Max-PlanckInstitute for Astrophysics (MPA), New Mexico State University, Ohio State University, University of Pittsburgh, University of Portsmouth, Princeton University, the US Naval Observatory, and the University of Washington. GALEX (Galaxy Evolution Explorer) is a NASA Small Explorer, launched in April 2003. We gratefully acknowledge NASA's support for construction, operation, and science analysis for the GALEX mission, developed in cooperation with the Centre National d'Etudes Spatiales (CNES) of France and the Korean Ministry of Science and Technology.

\section{References}

Abazajian, K. N., Adelman-McCarthy, J. K., Agüeros, M. A., et al. 2009, ApJS, 182,543

Alexander, D. M., \& Hickox, R. C. 2012, New Astron. Rev., 56, 93

Alonso-Herrero, A., Colina, L., Packham, C., et al. 2006a, ApJ, 652, L83

Alonso-Herrero, A., Rieke, G. H., Rieke, M. J., et al. 2006b, ApJ, 650, 835

Baldwin, J. A., Phillips, M. M., \& Terlevich, R. 1981, PASP, 93, 5

Beichman, C. A., Neugebauer, G., Habing, H. J., Clegg, P. E., \& Chester, T. J. 1988, Infrared astronomical satellite (IRAS) catalogs and atlases, Vol. 1: Explanatory supplement

Bigiel, F., Leroy, A., Walter, F., et al. 2008, AJ, 136, 2846

Blanc, G. A., Heiderman, A., Gebhardt, K., Evans, II, N. J., \& Adams, J. 2009, ApJ, 704, 842

Bouwens, R. J., Illingworth, G. D., Franx, M., \& Ford, H. 2007, ApJ, 670, 928

Bouwens, R. J., Illingworth, G. D., Oesch, P. A., et al. 2011, ApJ, 737, 90

Bouwens, R. J., Illingworth, G. D., Oesch, P. A., et al. 2015, ApJ, 803, 34

Brinchmann, J., Charlot, S., White, S. D. M., et al. 2004, MNRAS, 351, 1151

Brown, T. M., Ferguson, H. C., Davidsen, A. F., \& Dorman, B. 1997, ApJ, 482, 685

Brown, M. J. I., Moustakas, J., Smith, J.-D. T., et al. 2014, ApJS, 212, 18

Buat, V., Iglesias-Páramo, J., Seibert, M., et al. 2005, ApJ, 619, L51

Burgarella, D., Buat, V., \& Iglesias-Páramo, J. 2005, MNRAS, 360, 1413 Calzetti, D. 1997, in AIP Conf. Ser. 408, ed. W. H. Waller, 403

Calzetti, D. 2013, in Secular Evolution of Galaxies, eds. J. Falcón-Barroso, \& J. H. Knapen (CUP), 419

Calzetti, D., Armus, L., Bohlin, R. C., et al. 2000, ApJ, 533, 682

Calzetti, D., Kennicutt, R. C., Engelbracht, C. W., et al. 2007, ApJ, 666, 870

Calzetti, D., Wu, S.-Y., Hong, S., et al. 2010, ApJ, 714, 1256

Cardelli, J. A., Clayton, G. C., \& Mathis, J. S. 1989, ApJ, 345, 245

Castillo-Morales, A., Gallego, J., Pérez-Gallego, J., et al. 2011, MNRAS, 411, 1819

Castro, A., Miyaji, T., Shirahata, M., et al. 2014, PASJ, 66, 11020

Catalán-Torrecilla, C., Gil de Paz, A., Castillo-Morales, Á., Iglesias-Páramo, J., \& CALIFA Collaboration. 2014, in ASP Conf. Ser. 480, eds. M. S. Seigar, \& P. Treuthardt, 231

Charlot, S., \& Fall, S. M. 2000, ApJ, 539, 718

Chary, R., \& Elbaz, D. 2001, ApJ, 556, 562

Cluver, M. E., Jarrett, T. H., Hopkins, A. M., et al. 2014, ApJ, 782, 90

Cortese, L., Boselli, A., Franzetti, P., et al. 2008, MNRAS, 386, 1157

Dale, D. A., \& Helou, G. 2002, ApJ, 576, 159

de Vaucouleurs, G., de Vaucouleurs, A., Corwin, Jr., H. G., et al. 1991, Third Reference Catalogue of Bright Galaxies. Vol. I: Explanations and references. Volume II: Data for galaxies between $0 \mathrm{~h}$ and 12h, Vol. III: Data for galaxies between $12 \mathrm{~h}$ and $24 \mathrm{~h}$

Díaz-Santos, T., Alonso-Herrero, A., Colina, L., et al. 2008, ApJ, 685, 211

Díaz-Santos, T., Alonso-Herrero, A., Colina, L., et al. 2010, ApJ, 711, 328

Domínguez Sánchez, H., Bongiovanni, A., Lara-López, M. A., et al. 2014, MNRAS, 441, 2

Draine, B. T., \& Li, A. 2007, ApJ, 657, 810

Elbaz, D., Daddi, E., Le Borgne, D., et al. 2007, A\&A, 468, 33

Förster Schreiber, N. M., Genzel, R., Bouché, N., et al. 2009, ApJ, 706, 1364

Förster Schreiber, N. M., Shapley, A. E., Erb, D. K., et al. 2011a, ApJ, 731, 65 Förster Schreiber, N. M., Shapley, A. E., Genzel, R., et al. 2011b, ApJ, 739, 45

Gallego, J., Zamorano, J., Aragon-Salamanca, A., \& Rego, M. 1995, ApJ, 455, L1

Gallego, J., Zamorano, J., Rego, M., Alonso, O., \& Vitores, A. G. 1996, A\&AS, 120,323

García-Benito, R., Zibetti, S., Sánchez, S. F., et al. 2015, A\&A, 576, A135

Genzel, R., Burkert, A., Bouché, N., et al. 2008, ApJ, 687, 59

Gil de Paz, A., Boissier, S., Madore, B. F., et al. 2007, ApJS, 173, 185

Gonzalez-Delgado, R. M., Perez, E., Diaz, A. I., et al. 1995, ApJ, 439, 604

Gordon, K. D., Clayton, G. C., Witt, A. N., \& Misselt, K. A. 2000, ApJ, 533, 236

Hao, C.-N., Kennicutt, R. C., Johnson, B. D., et al. 2011, ApJ, 741, 124

Hirashita, H., Buat, V., \& Inoue, A. K. 2003, A\&A, 410, 83

Hopkins, A. M., \& Beacom, J. F. 2006, ApJ, 651, 142 
Hummer, D. G., \& Storey, P. J. 1987, MNRAS, 224, 801

Husemann, B., Jahnke, K., Sánchez, S. F., et al. 2013, A\&A, 549, A87 Iglesias-Páramo, J., Buat, V., Takeuchi, T. T., et al. 2006, ApJS, 164, 38 Inoue, A. K., Hirashita, H., \& Kamaya, H. 2001, ApJ, 555, 613 Jarrett, T. H., Masci, F., Tsai, C. W., et al. 2013, AJ, 145, 6 Johnson, B. D., Schiminovich, D., Seibert, M., et al. 2007, ApJS, 173, 377 Jonsson, P., Groves, B. A., \& Cox, T. J. 2010, MNRAS, 403, 17 Kauffmann, G., Heckman, T. M., Tremonti, C., et al. 2003, MNRAS, 346, 1055 Kelz, A., Verheijen, M. A. W., Roth, M. M., et al. 2006, PASP, 118, 129 Kennicutt, R. C., \& Evans, N. J. 2012, ARA\&A, 50, 531 Kennicutt, Jr., R. C. 1998a, ARA\&A, 36, 189

Kennicutt, Jr., R. C. 1998b, ApJ, 498, 541

Kennicutt, Jr., R. C., Armus, L., Bendo, G., et al. 2003, PASP, 115, 928 Kennicutt, Jr., R. C., Calzetti, D., Walter, F., et al. 2007, ApJ, 671, 333

Kennicutt, Jr., R. C., Hao, C.-N., Calzetti, D., et al. 2009, ApJ, 703, 1672 Kong, X., Charlot, S., Brinchmann, J., \& Fall, S. M. 2004, MNRAS, 349, 769 Kroupa, P. 2001, MNRAS, 322, 231

Lang, P., Wuyts, S., Somerville, R. S., et al. 2014, ApJ, 788, 11 Leroy, A. K., Walter, F., Brinks, E., et al. 2008, AJ, 136, 2782

Leroy, A. K., Bigiel, F., de Blok, W. J. G., et al. 2012, AJ, 144, 3

Li, Y., Crocker, A. F., Calzetti, D., et al. 2013, ApJ, 768, 180

Lilly, S. J., Le Fevre, O., Hammer, F., \& Crampton, D. 1996, ApJ, 460, L1

Madau, P., \& Dickinson, M. 2014, ARA\&A, 52, 415

Madau, P., Ferguson, H. C., Dickinson, M. E., et al. 1996, MNRAS, 283, 1388

Marino, R. A., Gil de Paz, A., Castillo-Morales, A., et al. 2012, ApJ, 754, 61

Mármol-Queraltó, E., Sánchez, S. F., Marino, R. A., et al. 2011, A\&A, 534, A8

Martin, D. C., Fanson, J., Schiminovich, D., et al. 2005, ApJ, 619, L1

Meurer, G. R., Heckman, T. M., Leitherer, C., et al. 1995, AJ, 110, 2665

Monreal-Ibero, A., Relaño, M., Kehrig, C., et al. 2011, MNRAS, 413, 2242

Moshir, M., et al. 1990, in IRAS Faint Source Catalogue, version 2.00

Moshir, M., Kopman, G., \& Conrow, T. A. O. 1992, IRAS Faint Source Survey, Explanatory supplement version 2

Muñoz-Mateos, J. C., Gil de Paz, A., Boissier, S., et al. 2009, ApJ, 701, 1965

Nelson, E. J., van Dokkum, P. G., Brammer, G., et al. 2012, ApJ, 747, L28

Nelson, E. J., van Dokkum, P. G., Momcheva, I., et al. 2013, ApJ, 763, L16

Neugebauer, G., Habing, H. J., van Duinen, R., et al. 1984, ApJ, 278, L1

Osterbrock, D. E. 1989, Astrophysics of gaseous nebulae and active galactic nuclei (University of Minnesota, CA: University Science Books)

Pellegrini, E. W., Baldwin, J. A., \& Ferland, G. J. 2010, ApJS, 191, 160

Pérez-González, P. G., Gil de Paz, A., Zamorano, J., et al. 2003, MNRAS, 338, 525

Pérez-González, P. G., Kennicutt, Jr., R. C., Gordon, K. D., et al. 2006, ApJ, 648, 987

Pérez-González, P. G., Rieke, G. H., Villar, V., et al. 2008, ApJ, 675, 234

Prescott, M. K. M., Kennicutt, Jr., R. C., Bendo, G. J., et al. 2007, ApJ, 668, 182 Ramos Almeida, C., Levenson, N. A., Rodríguez Espinosa, J. M., et al. 2009, ApJ, 702, 1127

Relaño, M., Monreal-Ibero, A., Vílchez, J. M., \& Kennicutt, R. C. 2010, MNRAS, 402, 1635

Rieke, G. H., Alonso-Herrero, A., Weiner, B. J., et al. 2009, ApJ, 692, 556

Roth, M. M., Kelz, A., Fechner, T., et al. 2005, PASP, 117, 620

Salim, S., Rich, R. M., Charlot, S., et al. 2007, ApJS, 173, 267

Sánchez, S. F., Jahnke, K., Wisotzki, L., et al. 2004, ApJ, 614, 586

Sánchez, S. F., Rosales-Ortega, F. F., Kennicutt, R. C., et al. 2011, MNRAS, 410 , 313

Sánchez, S. F., Kennicutt, R. C., Gil de Paz, A., et al. 2012, A\&A, 538, A8

Sánchez-Blázquez, P., Peletier, R. F., Jiménez-Vicente, J., et al. 2006, MNRAS, 371,703

Schruba, A., Leroy, A. K., Walter, F., et al. 2011, AJ, 142, 37
Seibert, M., Martin, D. C., Heckman, T. M., et al. 2005, ApJ, 619, L55

Somerville, R. S., \& Davé, R. 2015, ARA\&A, 53, 51

Taylor, P., \& Kobayashi, C. 2015, MNRAS, 448, 1835

Treyer, M., Schiminovich, D., Johnson, B., et al. 2007, ApJS, 173, 256

Vazdekis, A., Sánchez-Blázquez, P., Falcón-Barroso, J., et al. 2010, MNRAS, 404,1639

Walcher, C. J., Wisotzki, L., Bekeraité, S., et al. 2014, A\&A, 569, A1

Wright, E. L., Eisenhardt, P. R. M., Mainzer, A. K., et al. 2010, AJ, 140, 1868

Wuyts, S., Förster Schreiber, N. M., Nelson, E. J., et al. 2013, ApJ, 779, 135

Yamamura, I., Makiuti, S., Ikeda, N., \& Fukuda. 2010, AKARI/FIS All-Sky Survey Bright Source Catalogue Version 1.0 Release Note

Yi, S., Demarque, P., \& Oemler, Jr., A. 1997, ApJ, 486, 201

1 Departamento de Astrofísica y CC. de la Atmósfera, Universidad Complutense de Madrid, 28040 Madrid, Spain

e-mail: ccatalan@ucm.es

2 Instituto de Astrofísica de Andalucía-CSIC, Glorieta de la Astronomía, 18008 Granada, Spain

3 Estación Experimental de Zonas Áridas (CSIC), Ctra. de Sacramento s/n, La Cañada, 04120 Almería, Spain

4 Instituto de Astronomía,Universidad Nacional Autonóma de México, A.P. 70-264, 04510, México, D.F.

5 Institute of Astronomy, University of Cambridge, Madingley Road, Cambridge, CB3 OHA, UK

6 CEI Campus Moncloa, UCM-UPM, Departamento de Astrofísica y CC. de la Atmósfera, Universidad Complutense de Madrid, 28040 Madrid, Spain

7 Leibniz-Institut für Astrophysik Potsdam (AIP), An der Sternwarte 16, 14482 Potsdam, Germany

8 European Southern Observatory, Karl-Schwarzschild-Str. 2, 85748 Garching b. München, Germany

9 Instituto de Cosmologia, Relatividade e Astrofísica - ICRA, Centro Brasileiro de Pesquisas Físicas, Rua Dr.Xavier Sigaud 150, CEP 22290-180 Rio de Janeiro, RJ, Brazil

10 European Southern Observatory, Casilla 19001, Santiago 19, Chile

11 Sydney Institute for Astronomy, School of Physics A28, University of Sydney, NSW2006, Australia

12 Astronomical Institute of the Ruhr-University Bochum Universitaetsstr, 150, 44801 Bochum, Germany

13 Millennium Institute of Astrophysics, Universidad de Chile, Casilla 36-D, Santiago, Chile

14 Departamento de Astronomía, Universidad de Chile, Casilla 36-D, Santiago, Chile

15 Instituto de Astrofísica e Ciências do Espaço, Universidade do Porto, CAUP, Rua das Estrelas, 4150-762 Porto, Portugal

16 Australian Astronomical Observatory, PO Box 915, North Ryde, NSW 1670, Australia

17 Department of Physics and Astronomy, Macquarie University, NSW 2109, Australia

18 GEPI, Observatoire de Paris, CNRS UMR8111, Université Paris Diderot, Place Jules Janssen, 92190 Meudon, France

19 Centro de Estudios de la Física del Cosmos de Aragón (CEFCA), 44001 Teruel, Spain

20 Departamento de Física Teórica, Universidad Autónoma de Madrid, Cantoblanco, 28049, Spain 
C. Catalán-Torrecilla et al.: Star formation in the local Universe from the CALIFA sample. I.

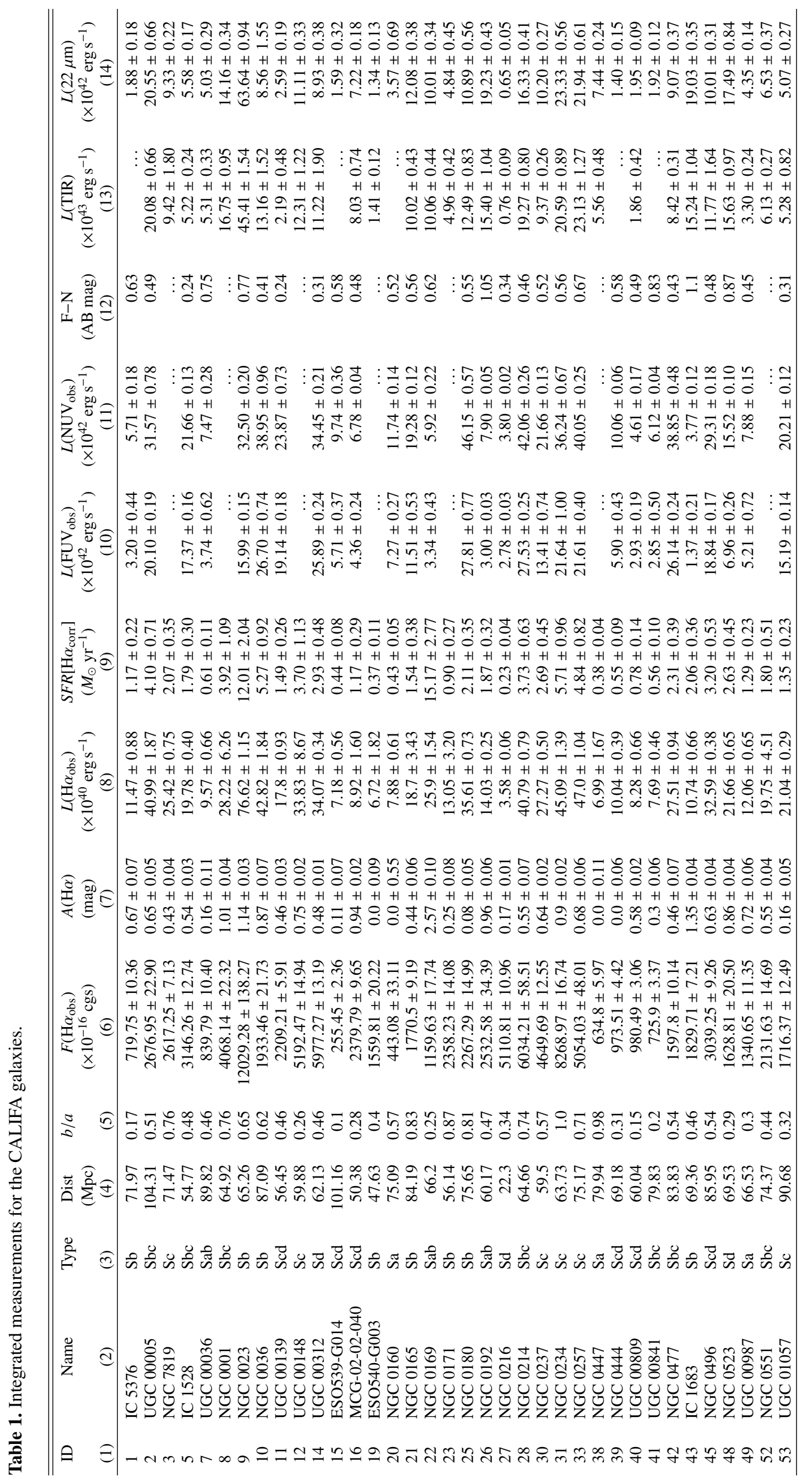

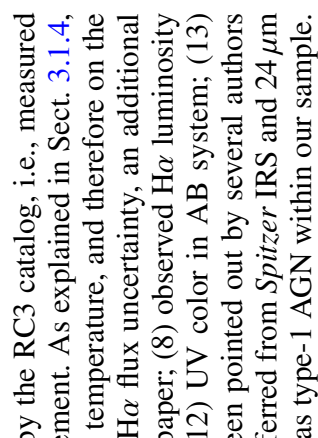

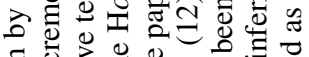

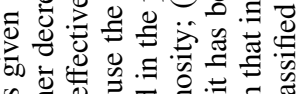

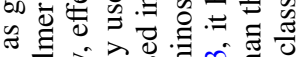

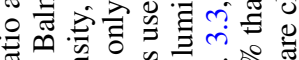

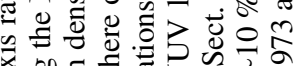

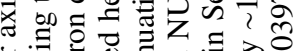

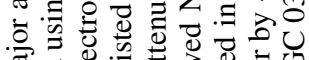

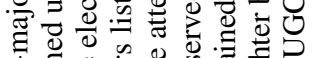
完. 自市 on

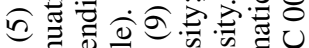

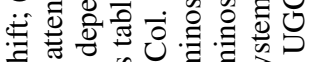

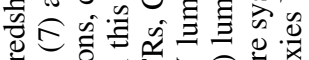

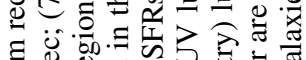

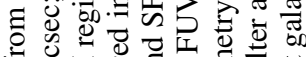

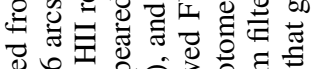

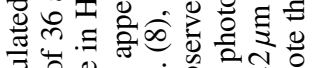
उ 응 ฮ.

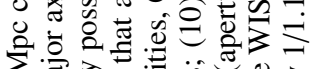

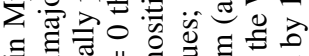
. ․ㅠ ․ㅠ

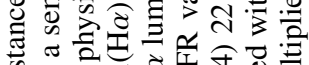

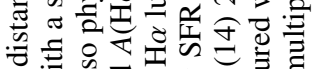

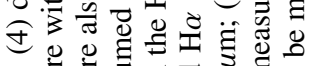
o

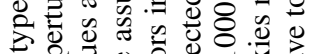

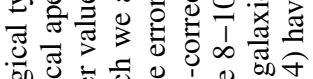
药.

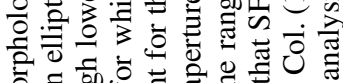

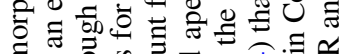

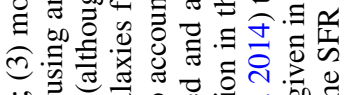

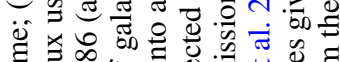

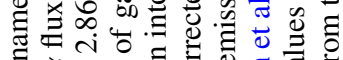

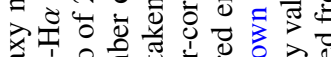

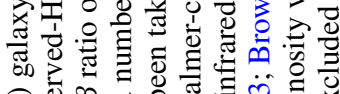
वิ

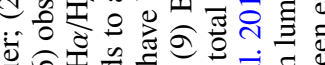

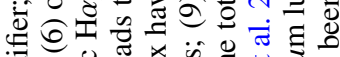

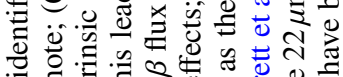

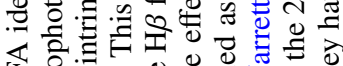

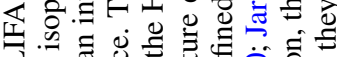

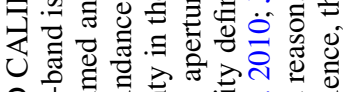

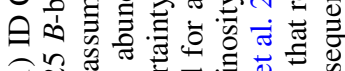
ड人

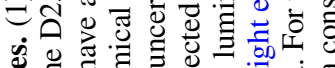

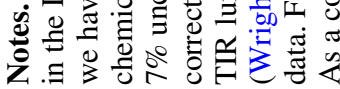


A\&A 584, A87 (2015)

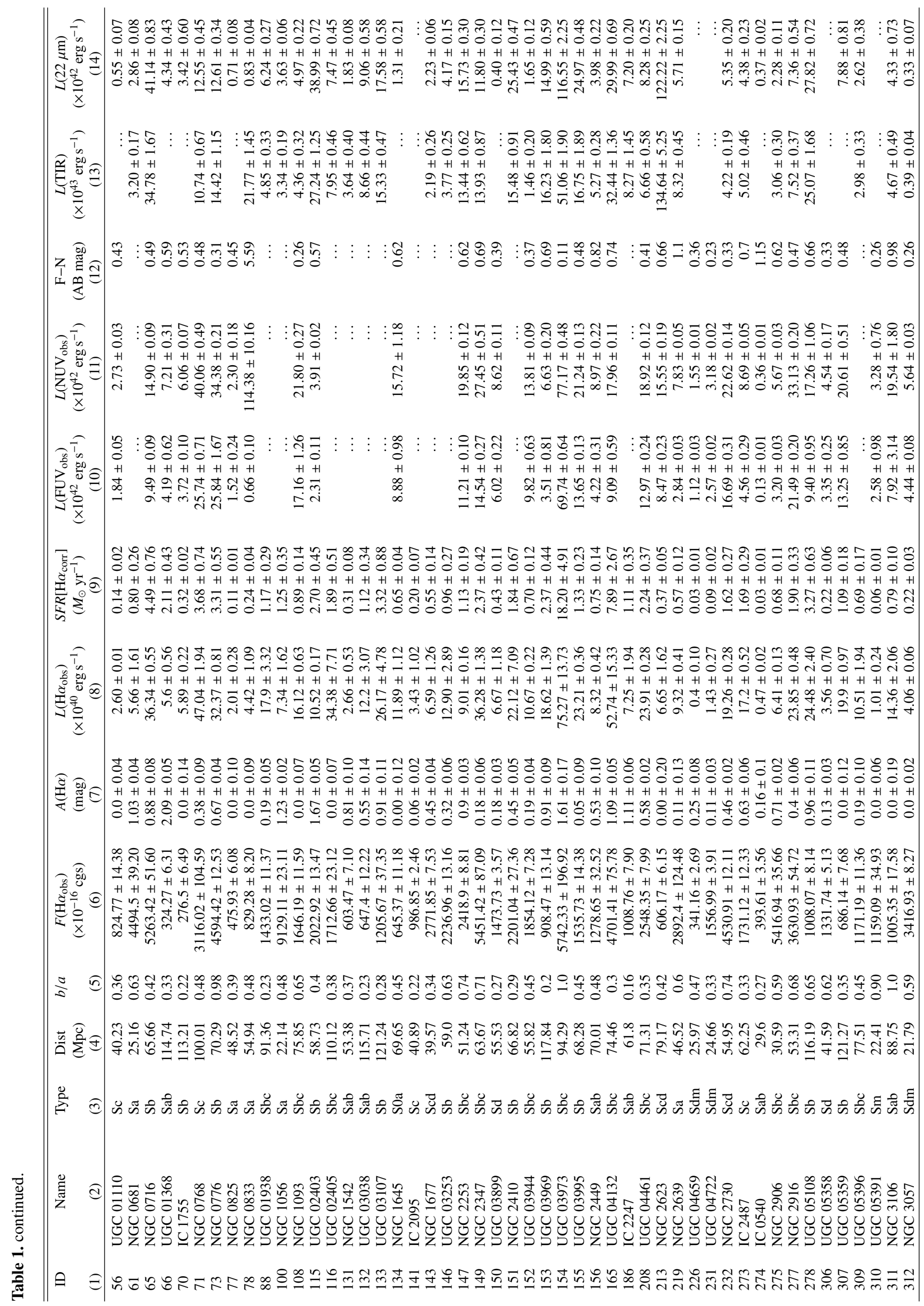


C. Catalán-Torrecilla et al.: Star formation in the local Universe from the CALIFA sample. I.

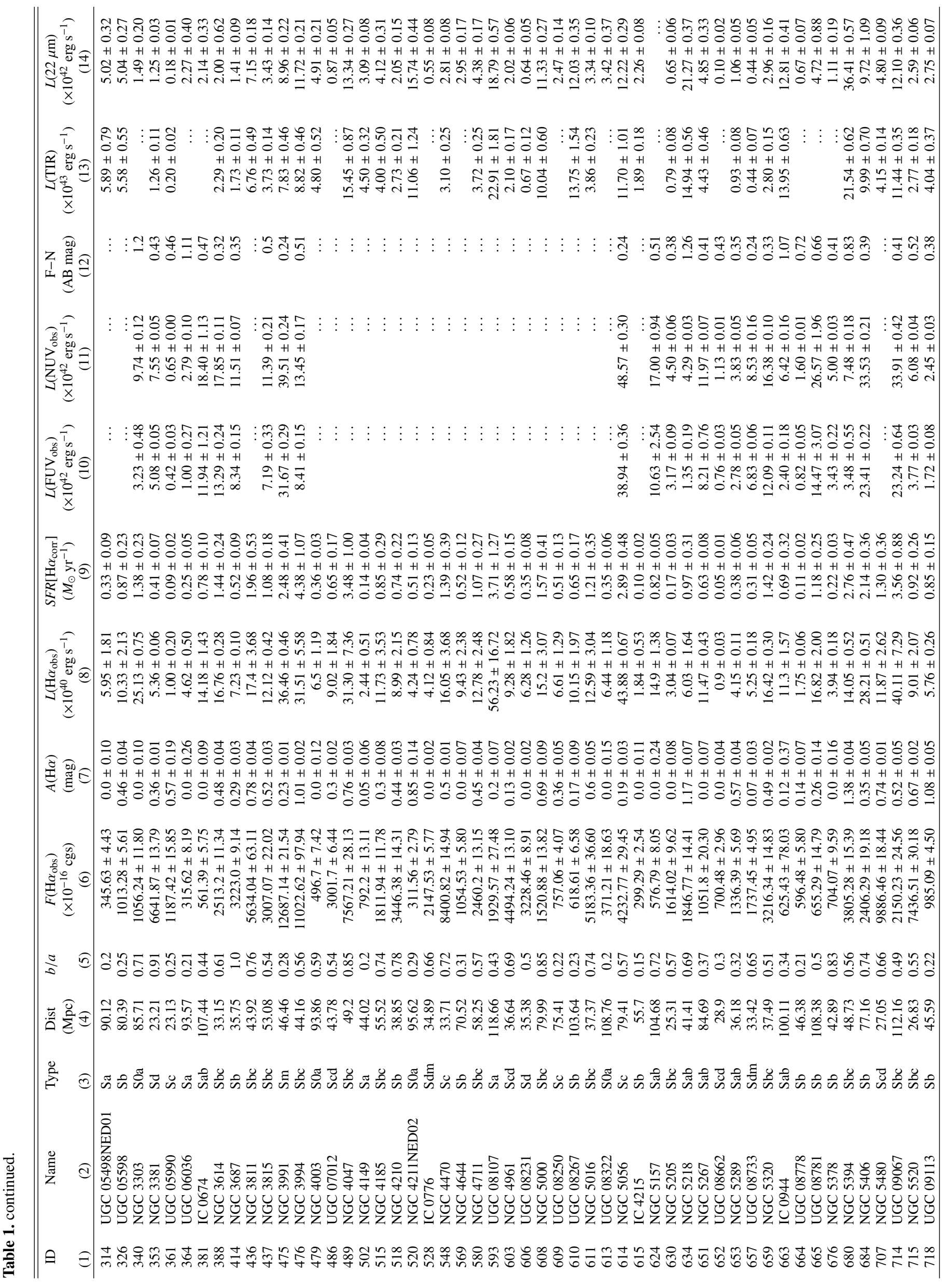


A\&A 584, A87 (2015)

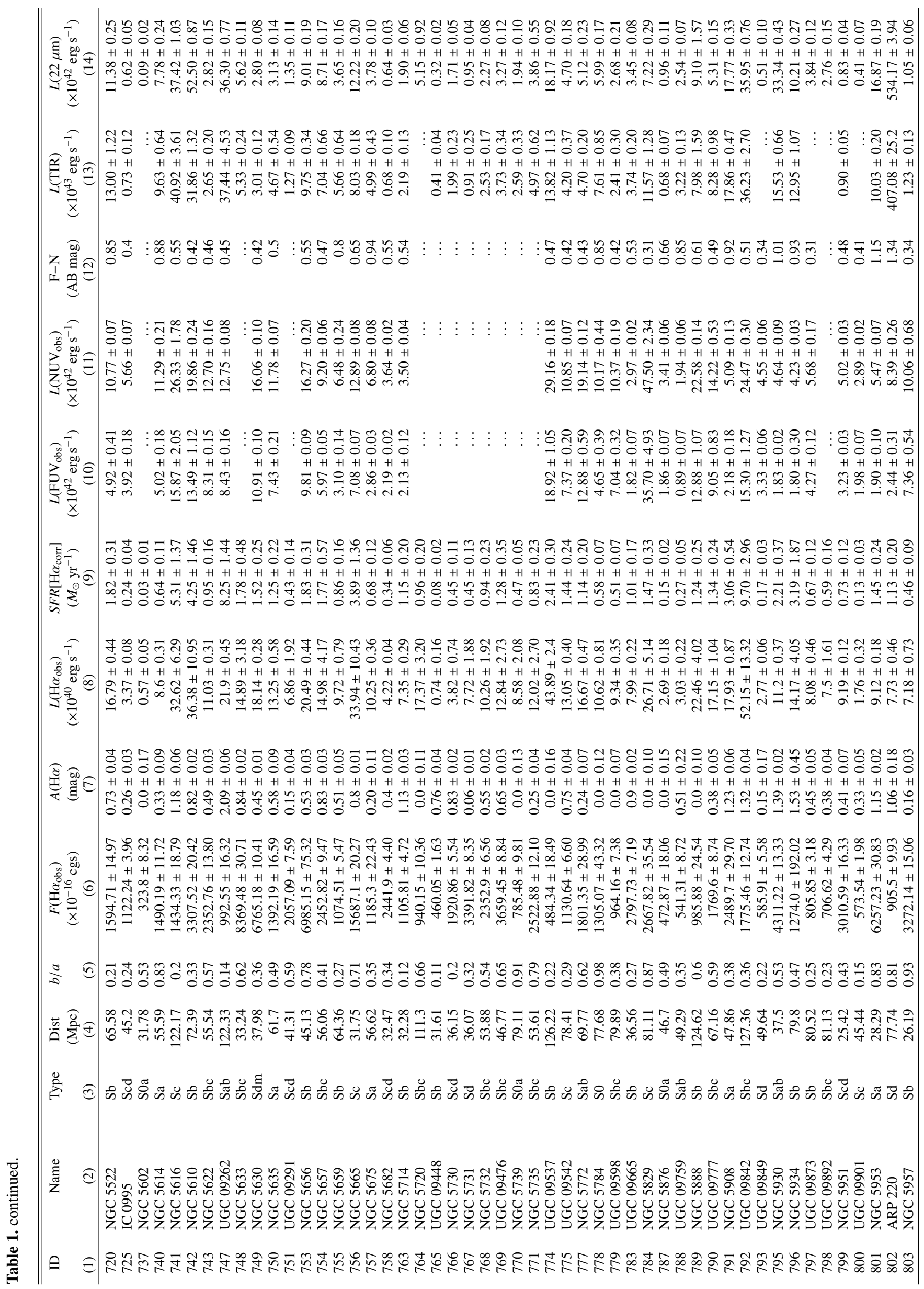


C. Catalán-Torrecilla et al.: Star formation in the local Universe from the CALIFA sample. I.

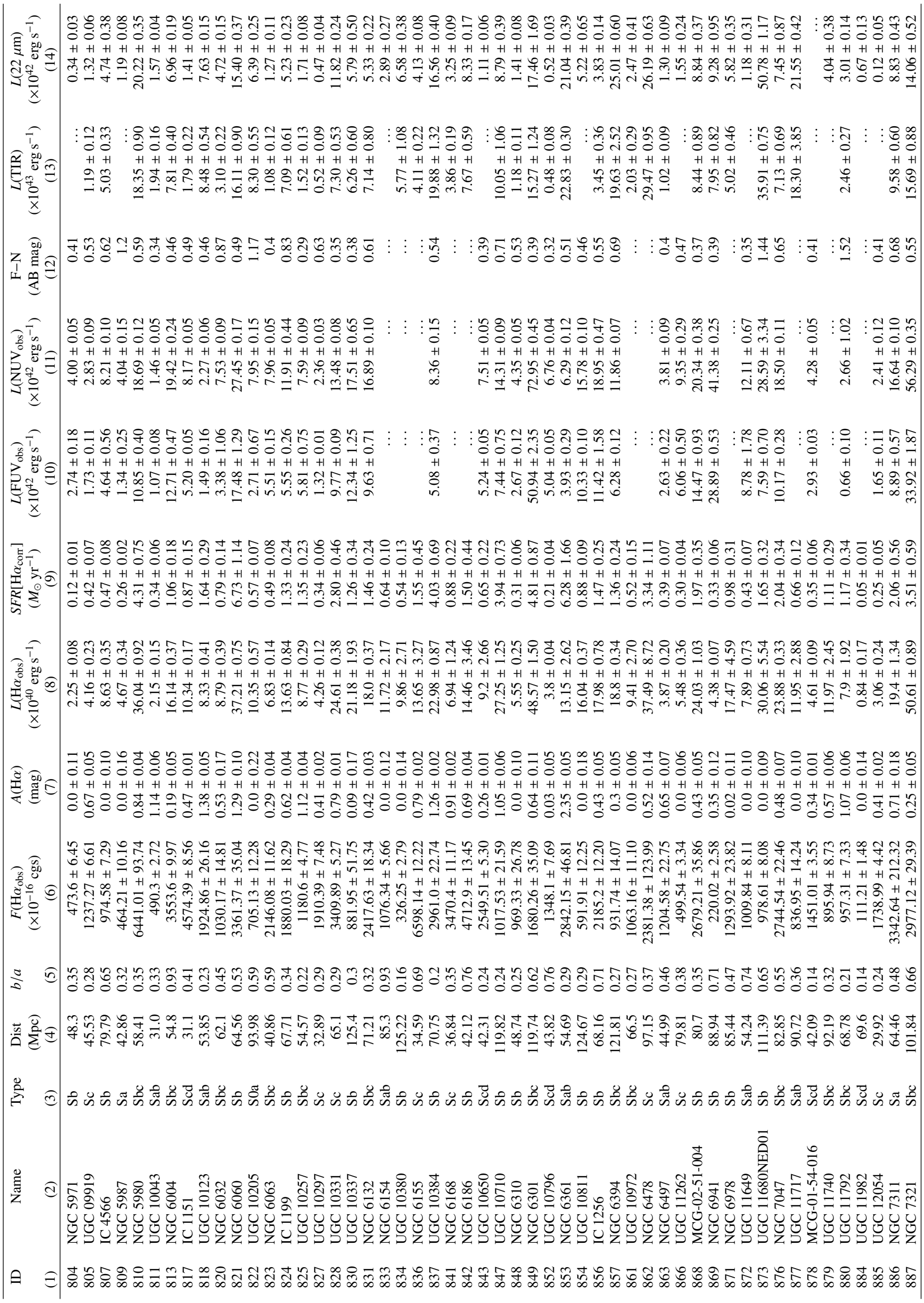


A\&A 584, A87 (2015)



